\title{
Search for new phenomena in final states with two opposite-charge, same-flavor leptons, jets, and missing transverse momentum in pp collisions at $\sqrt{s}=13 \mathrm{TeV}$
}

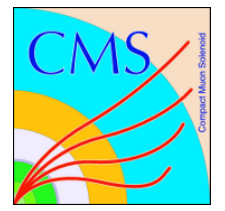

\section{The CMS collaboration}

\section{E-mail: cms-publication-committee-chair@cern.ch}

ABSTRACT: Search results are presented for physics beyond the standard model in final states with two opposite-charge, same-flavor leptons, jets, and missing transverse momentum. The data sample corresponds to an integrated luminosity of $35.9 \mathrm{fb}^{-1}$ of protonproton collisions at $\sqrt{s}=13 \mathrm{TeV}$ collected with the CMS detector at the LHC in 2016. The analysis uses the invariant mass of the lepton pair, searching for a kinematic edge or a resonant-like excess compatible with the $\mathrm{Z}$ boson mass. The search for a kinematic edge targets production of particles sensitive to the strong force, while the resonance search targets both strongly and electroweakly produced new physics. The observed yields are consistent with the expectations from the standard model, and the results are interpreted in the context of simplified models of supersymmetry. In a gauge mediated supersymmetry breaking (GMSB) model of gluino pair production with decay chains including $\mathrm{Z}$ bosons, gluino masses up to $1500-1770 \mathrm{GeV}$ are excluded at the $95 \%$ confidence level depending on the lightest neutralino mass. In a model of electroweak chargino-neutralino production, chargino masses as high as $610 \mathrm{GeV}$ are excluded when the lightest neutralino is massless. In GMSB models of electroweak neutralino-neutralino production, neutralino masses up to $500-650 \mathrm{GeV}$ are excluded depending on the decay mode assumed. Finally, in a model with bottom squark pair production and decay chains resulting in a kinematic edge in the dilepton invariant mass distribution, bottom squark masses up to $980-1200 \mathrm{GeV}$ are excluded depending on the mass of the next-to-lightest neutralino.

KEYwords: Hadron-Hadron scattering (experiments), Supersymmetry, Beyond Standard Model, Lepton production

ARXIV EPRINT: 1709.08908 


\section{Contents}

1 Introduction 1

2 Signal models $\quad 2$

3 The CMS detector 4

4 Data sets, triggers, and object selection 5

5 Signal regions $\quad 7$

6 Standard model background predictions $\quad 9$

$\begin{array}{ll}6.1 \text { Flavor-symmetric backgrounds } & 10\end{array}$

6.2 Drell-Yan+jets backgrounds 12

$\begin{array}{lll}\text { 6.3 Backgrounds with Z bosons plus genuine } p_{\mathrm{T}}^{\text {miss }} & 14\end{array}$

$\begin{array}{lll}7 & \text { Kinematic fit } & 14\end{array}$

8 Results 16

8.1 Results of the search in the on-Z signal regions 16

$\begin{array}{ll}8.2 \text { Results of the edge search } & 16\end{array}$

9 Interpretation $\quad \mathbf{2 2}$

9.1 Systematic uncertainty in the signal yield 22

9.2 Interpretations using simplified models 23

$\begin{array}{ll}10 \text { Summary } & 27\end{array}$

A Correlation and covariance matrices for the background predictions $\quad 28$

$\begin{array}{ll}\text { The CMS collaboration } & 36\end{array}$

\section{Introduction}

Supersymmetry (SUSY) [1-8] is a well-studied extension of the standard model (SM) and assumes a new fundamental symmetry that assigns a fermion (boson) to each SM boson (fermion). Supersymmetry resolves the hierarchy problem by stabilizing the Higgs boson (H) mass via additional quantum loop corrections from the top quark superpartner (top squark), which compensate for the large correction due to the top quark. If $R$-parity [9] is conserved, the lightest SUSY particle (LSP) predicted by the theory is stable and potentially massive, providing a candidate for the observed dark matter. Many SUSY models also lead to the unification of the electroweak (EW) and strong forces at high energies [10, 11]. 
This paper presents a search for signatures of new physics in events with two oppositecharge, same-flavor (OCSF) leptons (electrons or muons), jets, and missing transverse momentum. Interpretations of the search results are given in terms of simplified supersymmetric model spectra. The data set of proton-proton collisions used for this search was collected in 2016 with the CMS detector at the CERN LHC at a center-of-mass energy of $\sqrt{s}=13 \mathrm{TeV}$ and corresponds to an integrated luminosity of $35.9 \mathrm{fb}^{-1}$. Final states including an OCSF dilepton pair can occur in SUSY models via the decay of the superpartner of the SM neutral gauge bosons, the neutralino, when a heavier neutralino decays to a lighter neutralino LSP, or when the lightest neutralino is the next-to-lightest SUSY particle decaying to a gravitino LSP. Depending on the model parameters, the neutralino can decay into the LSP and either an on-shell Z boson or a virtual $\mathrm{Z} / \gamma^{*}$ boson, with the boson decaying to two charged leptons. The neutralino can also possibly decay into a lepton and its supersymmetric partner (slepton), the latter decaying into another lepton and the LSP. Decays involving an on-shell Z boson are expected to produce an excess of events in which the dilepton invariant mass is compatible with the $\mathrm{Z}$ boson mass, referred to as the "on-Z signature", while decays involving off-shell Z bosons or sleptons are expected to produce a characteristic edge shape in the invariant mass distribution of the dilepton system $\left(m_{\ell \ell}\right)[12]$, denoted as the "edge signature".

This search targets both the on-Z and edge signatures. For the on-Z signature, search regions are optimized separately depending on whether we target strong or EW SUSY production. In the case of strong production, the neutralino is part of a decay chain starting from a gluino or squark, while in the EW case, it is directly produced. The search for a kinematic edge is only performed under the assumption of strong SUSY production.

Searches for SUSY in these final states were performed previously by the CMS [13-18] and ATLAS [19-21] Collaborations. The CMS Collaboration reported the presence of an excess with an edge shape located at $m_{\ell \ell}=78.7 \pm 1.4 \mathrm{GeV}$ and with a local significance of 2.4 standard deviations (s.d.) in the data set collected at a center-of-mass-energy of $\sqrt{s}=8 \mathrm{TeV}$ [13]. The ATLAS Collaboration did not confirm this excess in its $\sqrt{s}=8 \mathrm{TeV}$ dataset, but reported a resonant-like excess of events compatible with the $\mathrm{Z}$ boson mass and with a local significance of 3.0 s.d. [19]. Neither of these excesses were confirmed in the data sets collected at a center-of-mass-energy of $\sqrt{s}=13 \mathrm{TeV}$ during 2015 by the CMS Collaboration [14] and during 2015 and the first half of 2016 by the ATLAS Collaboration [21].

\section{Signal models}

The results of this search are interpreted in the context of various simplified models of SUSY [22-26], as described below. In all models, the W, Z, and Higgs bosons are assumed to decay according to their SM branching fractions.

This search is designed to be sensitive to both strong and EW SUSY production leading to the on- $\mathrm{Z}$ signature. Most of the simplified models used for interpretation of the on- $\mathrm{Z}$ results represent gauge-mediated supersymmetry breaking (GMSB) models [27-29]. The first of these GMSB models assumes strong production of a pair of gluinos $(\widetilde{\mathrm{g}})$ that each decays into a pair of quarks $(\mathrm{u}, \mathrm{d}, \mathrm{s}, \mathrm{c}$, or $\mathrm{b})$ and the lightest neutralino, $\widetilde{\chi}_{1}^{0}$. The $\widetilde{\chi}_{1}^{0}$ 
in turn decays into a massless gravitino $(\widetilde{\mathrm{G}})$ and an on-shell $\mathrm{Z}$ boson. The decay chain corresponding to this gluino GMSB model is shown in figure 1 (upper left).

The three other models used for the on-Z signature assume EW production. The upper right diagram in figure 1 corresponds to chargino-neutralino $\left(\widetilde{\chi}_{1}^{ \pm}-\widetilde{\chi}_{2}^{0}\right)$ production, with $\widetilde{\chi}_{1}^{ \pm}$ decaying to a $\mathrm{W}$ boson and the LSP, $\widetilde{\chi}_{1}^{0}$, while the next-to-lightest neutralino, $\widetilde{\chi}_{2}^{0}$, decays to a $\mathrm{Z}$ boson and $\widetilde{\chi}_{1}^{0}$. The production cross sections for this model are computed in a limit of mass-degenerate wino $\widetilde{\chi}_{1}^{ \pm}$and $\widetilde{\chi}_{2}^{0}$, and light bino $\widetilde{\chi}_{1}^{0}$, with all the other sparticles assumed to be heavy and decoupled. Gauge mediated supersymmetry breaking is not assumed for this WZ model, and the $\widetilde{\chi}_{1}^{0}$ is allowed to be massive.

The remaining two models considered assume the production of neutralino-neutralino $\left(\widetilde{\chi}_{1}^{0}-\widetilde{\chi}_{1}^{0}\right)$ pairs in GMSB. For bino- or wino-like neutralinos, the neutralino pair production cross section is very small, and thus we consider a specific GMSB model with massdegenerate higgsinos $\widetilde{\chi}_{1}^{ \pm}, \widetilde{\chi}_{2}^{0}$, and $\widetilde{\chi}_{1}^{0}$ as the next-to-lightest SUSY particles and a massless gravitino as the LSP [27-29]. In the production of any two of these, $\widetilde{\chi}_{1}^{ \pm}$or $\widetilde{\chi}_{2}^{0}$ decays immediately to $\widetilde{\chi}_{1}^{0}$ and low-momentum particles that do not impact the analysis, effectively yielding pair production of $\widetilde{\chi}_{1}^{0} \widetilde{\chi}_{1}^{0}$. Intermediate production of either $\widetilde{\chi}_{1}^{ \pm}$or $\widetilde{\chi}_{2}^{0}$ is therefore not explicitly shown in the lower two diagrams of figure 1 representing these models. In the first model (lower left of figure 1), the only allowed decay of the lightest neutralino is to a $\mathrm{Z}$ boson and a massless gravitino. In the other model (lower two diagrams of figure 1), the lightest neutralino is allowed to decay to a gravitino and either a $\mathrm{Z}$ boson or an SM-like Higgs boson, with a $50 \%$ branching fraction to each decay channel. The cross sections for higgsino pair production are computed in a limit of mass-degenerate higgsino states $\widetilde{\chi}_{2}^{0}$, $\widetilde{\chi}_{1}^{ \pm}$, and $\widetilde{\chi}_{1}^{0}$, with all the other sparticles assumed to be heavy and decoupled. Following the convention of real mixing matrices and signed neutralino masses [30], we set the sign of the mass of $\widetilde{\chi}_{1}^{0}\left(\widetilde{\chi}_{2}^{0}\right)$ to $+1(-1)$. The lightest two neutralino states are defined as symmetric (anti-symmetric) combinations of higgsino states by setting the product of the elements $N_{i 3}$ and $N_{i 4}$ of the neutralino mixing matrix $N$ to $+0.5(-0.5)$ for $i=1(2)$. The elements $U_{12}$ and $V_{12}$ of the chargino mixing matrices $U$ and $V$ are set to 1 .

The signal model for the edge search, referred to as the slepton edge model, assumes the production of a pair of bottom squarks $(\widetilde{b})$, the superpartner of the bottom quark, where each decays to $\widetilde{\chi}_{2}^{0}$ and a bottom quark. Two decay modes of the $\widetilde{\chi}_{2}^{0}$ are considered, each with a $50 \%$ branching fraction; they are both illustrated in figure 2 . In the first mode, the $\widetilde{\chi}_{2}^{0}$ decays to a $\mathrm{Z}$ boson and $\widetilde{\chi}_{1}^{0}$, which is stable. The $\mathrm{Z}$ boson can be on- or off-shell, depending on the mass difference between the neutralinos. The second decay mode features sequential two-body decays with an intermediate slepton $\widetilde{\ell}(\widetilde{\mathrm{e}}, \widetilde{\mu}): \widetilde{\chi}_{2}^{0} \rightarrow \widetilde{\ell} \ell \rightarrow \ell \ell \widetilde{\chi}_{1}^{0}$. The masses of the sleptons are assumed to be degenerate and equal to the average of the $\widetilde{\chi}_{2}^{0}$ and $\widetilde{\chi}_{1}^{0}$ masses. The masses of the $\widetilde{\mathrm{b}}$ and $\widetilde{\chi}_{2}^{0}$ are free parameters, while the mass of $\widetilde{\chi}_{1}^{0}$ is fixed at $100 \mathrm{GeV}$. This scheme allows the position of the signal edge to vary in the invariant mass distribution depending on the mass difference between the $\widetilde{\chi}_{2}^{0}$ and $\widetilde{\chi}_{1}^{0}$. The mass of the $\widetilde{\chi}_{1}^{0}$ is chosen in such a way that the $\widetilde{\chi}_{2}^{0}$ mass is always greater by at least $50 \mathrm{GeV}$, setting the minimum possible edge position at $50 \mathrm{GeV}$. 

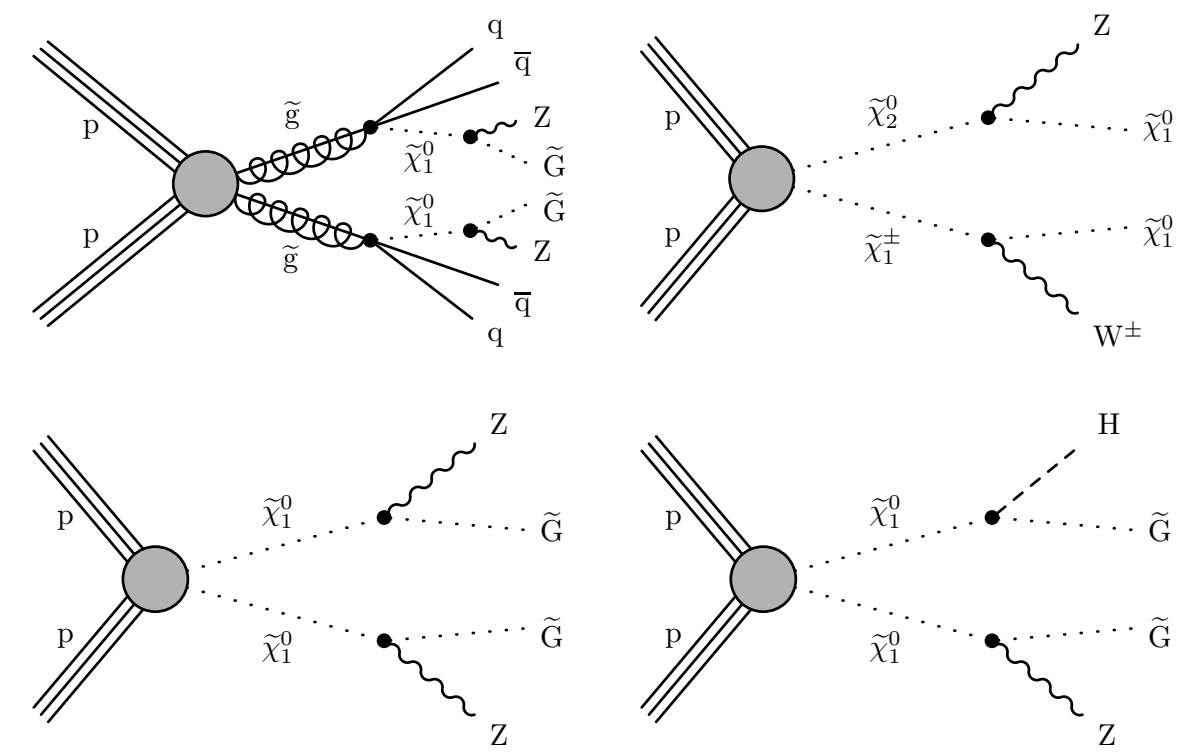

Figure 1. Diagrams for models with decays containing at least one dilepton pair stemming from an on-shell $\mathrm{Z}$ boson decay studied in this analysis. The model targeted by the strong-production search is shown in the upper left. The three other diagrams correspond to EW production of charginoneutralino or neutralino-neutralino pairs. All the diagrams containing a gravitino $(\widetilde{\mathrm{G}})$ represent gauge-mediated SUSY breaking (GMSB) models.

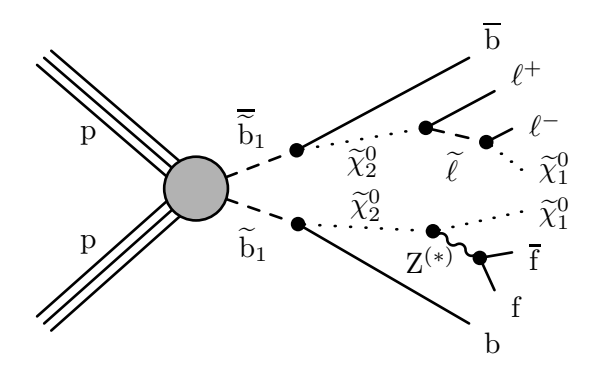

Figure 2. Diagram showing a possible decay chain in the slepton edge model. Bottom squarks are pair produced with subsequent decays that frequently contain dilepton pairs. This model features a characteristic edge in the $m_{\ell \ell}$ spectrum given approximately by the mass difference between the $\widetilde{\chi}_{2}^{0}$ and $\widetilde{\chi}_{1}^{0}$ particles.

\section{The CMS detector}

The central feature of the CMS apparatus is a superconducting solenoid, $13 \mathrm{~m}$ in length and $6 \mathrm{~m}$ in diameter, that provides an axial magnetic field of $3.8 \mathrm{~T}$. Within the solenoid volume are various particle detection systems. Charged-particle trajectories are measured by silicon pixel and strip trackers, covering $0<\phi<2 \pi$ in azimuth and $|\eta|<2.5$, where the pseudorapidity $\eta$ is defined as $-\log [\tan (\theta / 2)]$, with $\theta$ being the polar angle of the trajectory of the particle with respect to the counterclockwise beam direction. A crys- 
tal electromagnetic calorimeter (ECAL) and a brass and scintillator hadron calorimeter (HCAL) surround the tracking volume. The calorimeters provide energy and direction measurements of electrons and hadronic jets. Muons are detected in gas-ionization detectors embedded in the steel flux-return yoke outside the solenoid. The detector is nearly hermetic, allowing for momentum balance measurements in the plane transverse to the beam direction. A two-tier trigger system selects events of interest for physics analysis. A more detailed description of the CMS detector, together with a definition of the coordinate system used and the relevant kinematic variables, can be found in ref. [31].

\section{Data sets, triggers, and object selection}

This analysis uses data samples of $\mathrm{e}^{ \pm} \mathrm{e}^{\mp}$ and $\mu^{ \pm} \mu^{\mp}$ events for the signal region (SR) selections and $\mathrm{e}^{ \pm} \mu^{\mp}$ events for control regions (CRs). Events are collected with a set of dilepton (ee, $\mu \mu$, or $\mathrm{e} \mu$ ) triggers that require the magnitude of the transverse momentum $p_{\mathrm{T}}>17$ or $23 \mathrm{GeV}$ for the highest $p_{\mathrm{T}}$ lepton, depending on the data taking period, except for the dimuon trigger where the requirement is always $p_{\mathrm{T}}>17 \mathrm{GeV}$. These triggers impose loose isolation criteria on the leptons. For the next-to-highest $p_{\mathrm{T}}$ electron (muon), $p_{\mathrm{T}}>12$ (8) $\mathrm{GeV}$ is required, and electrons (muons) must satisfy $|\eta|<2.5$ (2.4). In order to retain high signal efficiency, in particular for highly boosted dilepton systems, dilepton triggers without an isolation requirement are also used. These require $p_{\mathrm{T}}>33 \mathrm{GeV}$ for both leptons in the dielectron case and $p_{\mathrm{T}}>30 \mathrm{GeV}$ for both leptons in the electron-muon case. In the dimuon case, they require either $p_{\mathrm{T}}>27$ (8) or $p_{\mathrm{T}}>30$ (11) GeV for the highest (next-to-highest) $p_{\mathrm{T}}$ muon depending on the data taking period. The trigger efficiencies are measured in data using events selected by a suite of jet triggers and are found to be $90-96 \%$.

The particle-flow (PF) event algorithm [32] reconstructs and identifies particle candidates in the event, referred to as PF objects. To select collision events we require at least one reconstructed vertex. The reconstructed vertex with the largest value of summed physicsobject $p_{\mathrm{T}}^{2}$ is taken to be the primary pp interaction vertex. The physics objects used for the primary vertex selection are the objects returned by a jet finding algorithm [33, 34] applied to all charged tracks associated with the vertex, plus the corresponding associated missing transverse momentum. The missing transverse momentum vector $\vec{p}_{\mathrm{T}}^{\text {miss }}$ is defined as the projection onto the plane perpendicular to the beam axis of the negative vector sum of the momenta of all reconstructed PF objects in an event. Its magnitude is referred to as $p_{\mathrm{T}}^{\text {miss }}$.

Electrons, reconstructed by associating tracks with ECAL clusters, are identified using a multivariate approach based on information on the cluster shape in the ECAL, track reconstruction quality, and the matching between the track and the ECAL cluster [35]. Electrons from reconstructed photon conversions are rejected. Muons are reconstructed from tracks found in the muon system associated with tracks found in the tracker. They are identified based on the quality of the track fit and the number of associated hits in the tracking detectors. For both lepton flavors, the impact parameter with respect to the primary vertex is required to be within $0.5 \mathrm{~mm}$ in the transverse plane and less than $1 \mathrm{~mm}$ along the beam direction. The lepton isolation variable is defined as the scalar $p_{\mathrm{T}}$ sum of all PF objects in a cone around the lepton (excluding those identified as electrons or 
muons). To mitigate the impact of additional pp interactions in the same or nearby bunch crossings (pileup), only charged PF objects compatible with the primary vertex are included in the sum, and the average expected pileup contribution is subtracted from the neutral component of the isolation. The isolation sum is required to be smaller than 10 (20)\% of the lepton $p_{\mathrm{T}}$ for electrons (muons). The cone size varies with lepton $p_{\mathrm{T}}$ and is chosen to be $\sqrt{(\Delta \phi)^{2}+(\Delta \eta)^{2}}=\Delta R=0.2$ for $p_{\mathrm{T}}<50 \mathrm{GeV}, \Delta R=10 \mathrm{GeV} / p_{\mathrm{T}}$ for $50<p_{\mathrm{T}}<200 \mathrm{GeV}$, and $\Delta R=0.05$ for $p_{\mathrm{T}}>200 \mathrm{GeV}$. This shrinking cone size with increasing $p_{\mathrm{T}}$ preserves high efficiency for leptons from Lorentz-boosted boson decays [36]. To identify events with three or more charged leptons, additional leptons beyond the first two are selected with the looser requirement for the isolation sum to be less than $40 \%$ of the lepton $p_{\mathrm{T}}$.

Photons are required to pass identification criteria based on the cluster shape in the ECAL and the fraction of energy deposited in the HCAL [37]. Photons must satisfy $p_{\mathrm{T}}>25 \mathrm{GeV}$, and be within $|\eta|<2.4$, excluding the "transition region" of $1.4<|\eta|<1.6$ between the ECAL barrel and endcap. Photons are required to be isolated from other PF objects within a cone of $\Delta R=0.3$. To ensure the photon is well measured, it is required that $\Delta \phi\left(\vec{p}_{\mathrm{T}}^{\text {miss }}, \vec{p}_{\mathrm{T}}^{\gamma}\right)>0.4$. To distinguish photons from electrons, the photon is rejected if it can be connected to a pattern of hits in the pixel detector that indicate the presence of a charged particle track.

Isolated, charged-particle tracks identified by the PF algorithm are selected with looser requirements on a similar set of criteria to the leptons defined above and are used as a veto on the presence of additional charged leptons. When selecting charged PF objects, a track-based relative isolation is used. The relative track isolation is calculated using all charged PF objects within a cone $\Delta R=0.3$ and longitudinal impact parameter $|\Delta z|<$ $0.1 \mathrm{~cm}$ relative to the primary vertex. Particle-flow objects identified as electrons or muons (charged hadrons) are required to have $p_{\mathrm{T}}>5(10) \mathrm{GeV}$ and an isolation value less than $20(10) \%$ of the object $p_{\mathrm{T}}$.

Jets are clustered from PF objects, excluding charged hadrons not associated with the primary vertex, using the anti- $k_{\mathrm{T}}$ clustering algorithm [33] with a distance parameter of 0.4, implemented in the FASTJET package [34, 38]. Jets are required to satisfy $|\eta|<2.4$ and $p_{\mathrm{T}}>35 \mathrm{GeV}$, where the $p_{\mathrm{T}}$ is corrected for nonuniform detector response and multiple collision (pileup) effects [39, 40]. A jet is removed from the event if it lies within $\Delta R<0.4$ of any of the selected leptons or the highest $p_{\mathrm{T}}$ photon. The scalar sum of all jet $p_{\mathrm{T}}$ is referred to as $H_{\mathrm{T}}$. Corrections to the jet energy are propagated to $p_{\mathrm{T}}^{\text {miss }}$ using the procedure developed in ref. [39]. Identification of jets originating from $b$ quarks is performed with the combined secondary vertex (CSVv2) algorithm [41], using the medium working point, for which the typical efficiency for b quarks is around $60-75 \%$ and the mistagging rate for light-flavor jets is around $1.5 \%$. Jets with a lower threshold of $p_{\mathrm{T}}>25 \mathrm{GeV}$ are considered, and selected jets are denoted as b-tagged jets.

Events are selected by requiring two OCSF leptons $\left(\mathrm{e}^{ \pm} \mathrm{e}^{\mp}\right.$ or $\left.\mu^{ \pm} \mu^{\mp}\right)$ with $p_{\mathrm{T}}>25$ (20) $\mathrm{GeV}$ for the highest (next-to-highest) $p_{\mathrm{T}}$ lepton and $|\eta|<2.4$ for both leptons. The distance between the leptons must satisfy $\Delta R>0.1$ to avoid reconstruction efficiency differences between electrons and muons in events with collinear leptons. To ensure symmetry in acceptance between electrons and muons, all leptons in the transition region between the 
barrel and endcap of the ECAL, $1.4<|\eta|<1.6$, are rejected. A control sample of lepton pairs with opposite charge and different flavor (OCDF), $\mathrm{e}^{ \pm} \mu^{\mp}$, is defined using the same lepton selection criteria. All the parameters above have been chosen in order to maximize the lepton selection efficiency while keeping the efficiencies similar for electrons and muons. Photon events are used to predict one of the main backgrounds of this analysis, and a data control sample is selected as described below in section 6.2. To be consistent with the photon $p_{\mathrm{T}}$ threshold applied in this control sample, we require the $p_{\mathrm{T}}$ of the dilepton system to be greater than $25 \mathrm{GeV}$.

While the main SM backgrounds are estimated using data control samples, simulated events are used to estimate systematic uncertainties and some SM background components as described below. Next-to-leading order (NLO) and next-to-NLO cross sections [42-47] are used to normalize the simulated background samples, while NLO plus next-to-leadinglogarithmic (NLL) calculations [48-50] are used for the signal samples. Simulated samples of Drell-Yan (DY) processes and photons produced in association with jets are generated with the MADGraPh5_amC@NLO2.3.3 event generator [42] to leading order (LO) precision, with up to four additional partons in the matrix element calculations, using the MLM matching scheme [51]. Simulated $\mathrm{t} \overline{\mathrm{t}} \mathrm{V}(\mathrm{V}=\mathrm{W}, \mathrm{Z})$ and VVV events are produced with the same generator to NLO precision. Other SM processes, such as $\mathrm{VV}$, $\mathrm{t} \overline{\mathrm{t}}$, and single top quark production, are simulated using POWHEG 2.0 [52]. The matrix element calculations performed with these generators are interfaced with PYTHIA 8.212 [53] for the simulation of parton showering and hadronization. The NNPDF3.0 parton distribution functions (PDF) [54] are used for all samples. The detector response is simulated with a GEANT4 model [55] of the CMS detector. The simulation of new-physics signals is performed using the MADGRAPH5_aMC@NLO program at LO precision, with up to two additional partons in the matrix element calculation. Events are then interfaced with PYTHIA 8.212 for fragmentation and hadronization, and further processed using the CMS fast simulation package [56]. Multiple pp interactions are superimposed on the hard collision, and the simulated samples are reweighted such that the number of collisions per bunch crossing accurately reflects the distribution observed in data. Corrections are applied to the simulated samples to account for differences between simulation and data in the trigger and reconstruction efficiencies.

\section{Signal regions}

The selections for all SRs, described below, are summarized in table 1.

The on-Z search regions are designed to achieve low backgrounds from SM processes, while maintaining sensitivity to a variety of new-physics models, not only the processes described in section 2. The dilepton invariant mass is required to be in the range $86<$ $m_{\ell \ell}<96 \mathrm{GeV}$, which is compatible with the $\mathrm{Z}$ boson mass. The events must contain at least two jets and satisfy $p_{\mathrm{T}}^{\text {miss }}>100 \mathrm{GeV}$. The two highest $p_{\mathrm{T}}$ jets in the event are required to have a separation in $\phi$ from $\vec{p}_{\mathrm{T}}^{\text {miss }}$ of at least 0.4 to reduce backgrounds where the $p_{\mathrm{T}}^{\text {miss }}$ in the event comes from jet mismeasurements. Events containing additional electrons (muons) with $p_{\mathrm{T}}>10 \mathrm{GeV},|\eta|<2.5(2.4)$, and passing the looser isolation criteria from section 4 , are rejected, as are events containing an isolated, charged PF candidate passing 
the selections described in that section. Multiple on-Z SRs are defined using these selection criteria as a baseline: the first set for the strong production search and two additional regions for EW production searches.

For the on-Z strong-production SRs, we make selections requiring a large level of hadronic activity in the event, which we expect in the decays of strongly coupled new particles. We define three SR categories: "SRA" ( $2-3$ jets), "SRB" ( $4-5$ jets), and "SRC" ( $\geq 6$ jets). These categories are further divided as having either zero or at least one btagged jet. The kinematic variable $M_{\mathrm{T} 2}[57,58]$ is used to reduce the background from t $\overline{\mathrm{t}}$ events. This variable was first introduced to measure the mass of pair-produced particles, each decaying to the same final state, consisting of a visible and an invisible particle. It is defined using $\vec{p}_{\mathrm{T}}^{\text {miss }}$ and two visible objects (leptons, jets, or combinations thereof) as:

$$
M_{\mathrm{T} 2}=\min _{\vec{p}_{\mathrm{T}}^{\text {miss }(1)}+\vec{p}_{\mathrm{T}}^{\text {miss }(2)}=\vec{p}_{\mathrm{T}}^{\text {miss }}}\left[\max \left(M_{\mathrm{T}}^{(1)}, M_{\mathrm{T}}^{(2)}\right)\right],
$$

where $\vec{p}_{\mathrm{T}}^{\text {miss }(i)}(i=1,2)$ are trial vectors obtained by decomposing $\vec{p}_{\mathrm{T}}^{\text {miss }}$. The transverse masses $M_{\mathrm{T}}^{(i)}=\sqrt{2 p_{\mathrm{T}}^{\text {vis }} p_{\mathrm{T}}^{\text {miss }(i)}[1-\cos (\Delta \phi)]}$, where $\Delta \phi$ is the angle between the transverse momentum of the visible object and $\vec{p}_{\mathrm{T}}^{\text {miss }(i)}$, are obtained by pairing either of these trial vectors with one of the two visible objects. The minimization is performed over all trial momenta satisfying the $\vec{p}_{\mathrm{T}}^{\text {miss }}$ constraint. When building $M_{\mathrm{T} 2}$ from the two selected leptons and $\vec{p}_{\mathrm{T}}^{\text {miss }}$, denoted $M_{\mathrm{T} 2}(\ell \ell)$, its distribution exhibits a sharp decline around the mass of the $\mathrm{W}$ boson for $t \bar{t}$ events and is therefore well suited to suppress this background. A requirement of $M_{\mathrm{T} 2}(\ell \ell)>80 \mathrm{GeV}$ ( $100 \mathrm{GeV}$ for events with at least one b-tagged jet) is imposed in order to suppress $t \bar{t}$ backgrounds. Requirements are then placed on $H_{\mathrm{T}}$ depending on the number of jets and on the presence or absence of a b-tagged jet in the event, indicated by the labels "b tag" and "b veto," respectively. Finally, each SR is divided into multiple bins in $p_{\mathrm{T}}^{\text {miss }}$, depending on the number of selected jets. The precise requirements are summarized in table 1.

The first EW on-Z search region is denoted the "VZ" region and is designed to be sensitive to signatures where a hadronically decaying $\mathrm{W}$ or $\mathrm{Z}$ boson is produced in conjunction with the leptonically decaying $\mathrm{Z}$ boson. In order to reduce the $\mathrm{t} \overline{\mathrm{t}}$ background, events with a b-tagged jet are removed, and we require $M_{\mathrm{T} 2}(\ell \ell)>80 \mathrm{GeV}$. The two jets in the event that are closest in $\phi$ are then required to have a dijet invariant mass $m_{\mathrm{jj}}<110 \mathrm{GeV}$ to be consistent with the hadronic decay of a $\mathrm{W}$ or $\mathrm{Z}$ boson. The SR is then divided into four bins in $p_{\mathrm{T}}^{\text {miss }}$ : 100-150, 150-250, 250-350, and > $350 \mathrm{GeV}$.

The second EW-production search region is denoted the "HZ" region and is designed to be sensitive to signatures where a Higgs boson is produced in conjunction with the leptonically decaying $\mathrm{Z}$ boson. We target Higgs bosons decaying to $\mathrm{b} \overline{\mathrm{b}}$, due to its dominant branching ratio, and we therefore require events to have exactly two b-tagged jets with an invariant mass, $m_{\mathrm{bb}}$, less than $150 \mathrm{GeV}$. In order to reduce the t $\overline{\mathrm{t}}$ background, a $M_{\mathrm{T} 2}$ variable is calculated using two combinations of one lepton and one b-tagged jet as the visible objects. Each lepton is paired with a b-tagged jet, and all combinations of $M_{\mathrm{T} 2}$ are calculated. The smallest value of $M_{\mathrm{T} 2}$ is used, denoted $M_{\mathrm{T} 2}(\ell \mathrm{b} \ell \mathrm{b})$. The distribution of $M_{\mathrm{T} 2}(\ell \mathrm{b} \ell \mathrm{b})$ has an endpoint at the top quark mass for $\mathrm{t} \overline{\mathrm{t}}$ events, and we require $M_{\mathrm{T} 2}(\ell \mathrm{b} \ell \mathrm{b})>200 \mathrm{GeV}$. The $\mathrm{SR}$ is then divided into three bins in $p_{\mathrm{T}}^{\text {miss }}: 100-150,150-250$, 
and $>250 \mathrm{GeV}$. Although the selections for the EW VZ and HZ regions are mutually exclusive, they are not necessarily exclusive with respect to the strong-production SR selections. For interpretations of the analysis results, either the strong-production or the EW regions are considered depending on the signal model.

The baseline SR in the edge search requires $m_{\ell \ell}>20 \mathrm{GeV}$, at least two jets, $p_{\mathrm{T}}^{\text {miss }}>$ $150 \mathrm{GeV}, M_{\mathrm{T} 2}(\ell \ell)>80 \mathrm{GeV}$, and the two jets with the highest $p_{\mathrm{T}}$ to have a separation in $\phi$ from $\vec{p}_{\mathrm{T}}^{\text {miss }}$ of at least 0.4 . A fit is performed in this baseline region to search for a kinematic edge in the $m_{\ell \ell}$ spectrum. A counting experiment is also performed in seven bins of $m_{\ell \ell}$, excluding the range used for the on- $\mathrm{Z}$ search. These are summarized in table 1 .

A likelihood discriminant is used to distinguish between events originating from dileptonically decaying top quark pairs and other sources. The observables used for the likelihood discriminator are $p_{\mathrm{T}}^{\text {miss }}$, the $p_{\mathrm{T}}$ of the dilepton system, $|\Delta(\phi)|$ between the leptons, and an observable called $\Sigma m_{\ell \mathrm{b}}$. The latter is the sum of the invariant masses of the two lepton and b-tagged jet systems, and should have an endpoint at $2 \sqrt{M(\mathrm{t})^{2}-M(\mathrm{~W})^{2}}$ for events resulting from top quark pairs. To calculate $\Sigma m_{\ell \mathrm{b}}$, all pairings of a lepton with a jet are considered, and the pairing with the minimum invariant mass is selected. This process is repeated for the remaining lepton and jets, and the sum of the invariant masses of the two lepton-jet pairs is defined as $\Sigma m_{\ell \mathrm{b}}$. If b-tagged jets are present, they are given priority in the calculation of both lepton-jet systems; i.e., if one or more b-tagged jets are present, $\Sigma m_{\ell \mathrm{b}}$ between the leptons and the b-tagged jet(s) is minimized first, and then the remaining (b-tagged) jets are considered for the minimization of the sum $\Sigma m_{\ell \mathrm{b}}$ of the second lepton. To calculate the likelihood discriminant, the probability density functions of the four observables are determined by fits in the different-flavor (DF) control sample using the same kinematic requirements as the same-flavor (SF) SR except removing the $M_{\mathrm{T} 2}(\ell \ell)$ selection. The respective fit functions are a sum of two exponential functions for $p_{\mathrm{T}}^{\text {miss }}$, a second-order polynomial for $|\Delta(\phi)|$, and a Crystal Ball (CB) function [59] for both the dilepton $p_{\mathrm{T}}$ and $\Sigma m_{\ell \mathrm{b}}$ distributions. A likelihood function is constructed, and its negative logarithm is taken as the discriminator value. Two categories of events are

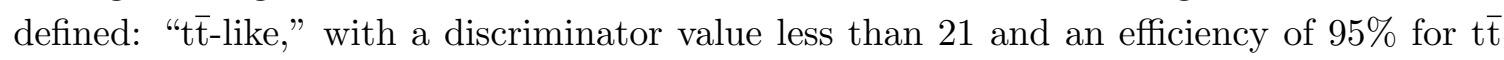

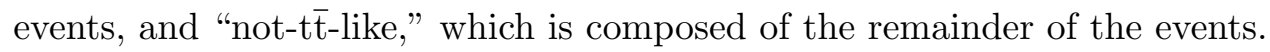

In addition, two aggregate SRs are defined for the edge search, integrating the mass bins below and above the $\mathrm{Z}$ boson mass for the not-t $\overline{\mathrm{t}}$-like category.

\section{$6 \quad$ Standard model background predictions}

The backgrounds from SM processes are divided into three categories. Those that produce DF pairs $\left(\mathrm{e}^{ \pm} \mu^{\mp}\right)$ as often as SF pairs $\left(\mu^{ \pm} \mu^{\mp}, \mathrm{e}^{ \pm} \mathrm{e}^{\mp}\right)$ are referred to as flavor-symmetric (FS) backgrounds. Among them, the dominant contribution arises from top quark pair production; subleading contributions are also present from $\mathrm{W}^{+} \mathrm{W}^{-}, \mathrm{Z} / \gamma^{*}(\rightarrow \tau \tau)$, tW single-top quark production, and leptons from hadron decays. Data samples of DF events are used to predict the SF background.

The remaining background categories contain flavor-correlated sources of lepton production that only contribute events with OCSF leptons. The dominant contributions at 


\begin{tabular}{|c|c|c|c|c|c|}
\hline \multicolumn{6}{|c|}{ Strong-production on-Z $\left(86<m_{\ell \ell}<96 \mathrm{GeV}\right)$ signal regions } \\
\hline Region & $N_{\text {jets }}$ & $N_{\text {b-jets }}$ & $H_{\mathrm{T}}[\mathrm{GeV}]$ & $M_{\mathrm{T} 2}(\ell \ell)[\mathrm{GeV}]$ & $p_{\mathrm{T}}^{\text {miss }}$ binning $[\mathrm{GeV}]$ \\
\hline SRA b veto & $2-3$ & $=0$ & $>500$ & $>80$ & $100-150,150-250,>250$ \\
\hline SRB b veto & $4-5$ & $=0$ & $>500$ & $>80$ & $100-150,150-250,>250$ \\
\hline SRC b veto & $\geq 6$ & $=0$ & - & $>80$ & $100-150,>150$ \\
\hline SRA b tag & $2-3$ & $\geq 1$ & $>200$ & $>100$ & $100-150,150-250,>250$ \\
\hline SRB b tag & $4-5$ & $\geq 1$ & $>200$ & $>100$ & $100-150,150-250,>250$ \\
\hline SRC b tag & $\geq 6$ & $\geq 1$ & - & $>100$ & $100-150,>150$ \\
\hline \multicolumn{6}{|c|}{ Electroweak-production on- $\mathrm{Z}\left(86<m_{\ell \ell}<96 \mathrm{GeV}\right)$ signal regions } \\
\hline Region & $N_{\text {jets }}$ & $N_{\text {b-jets }}$ & Dijet mass $[\mathrm{GeV}]$ & $M_{\mathrm{T} 2}[\mathrm{GeV}]$ & $p_{\mathrm{T}}^{\mathrm{miss}}$ binning $[\mathrm{GeV}]$ \\
\hline $\mathrm{VZ}$ & $\geq 2$ & $=0$ & $m_{\mathrm{jj}}<110$ & $M_{\mathrm{T} 2}(\ell \ell)>80$ & $100-150,150-250,250-350,>350$ \\
\hline $\mathrm{HZ}$ & $\geq 2$ & $=2$ & $m_{\mathrm{bb}}<150$ & $M_{\mathrm{T} 2}(\ell \mathrm{b} \ell \mathrm{b})>200$ & $100-150,150-250,>250$ \\
\hline \multicolumn{6}{|c|}{ Edge signal regions } \\
\hline Region & $N_{\text {jets }}$ & $p_{\mathrm{T}}^{\text {miss }}[\mathrm{GeV}]$ & $M_{\mathrm{T} 2}(\ell \ell)[\mathrm{GeV}]$ & tē likelihood & $m_{\ell \ell}$ binning $[\mathrm{GeV}]$ \\
\hline Edge fit & $\geq 2$ & $>150$ & $>80$ & - & $>20$ \\
\hline t't-like & $\geq 2$ & $>150$ & $>80$ & $<21$ & $\begin{array}{l}20-60,60-86,96-150,150-200, \\
200-300,300-400,>400\end{array}$ \\
\hline not-tt'-like & $\geq 2$ & $>150$ & $>80$ & $>21$ & 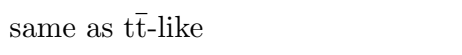 \\
\hline aggregate & $\geq 2$ & $>150$ & $>80$ & $>21$ & $20-86,>96$ \\
\hline
\end{tabular}

Table 1. Summary of all SR selections.

lower $p_{\mathrm{T}}^{\text {miss }}$ are from DY production in association with jets, where $p_{\mathrm{T}}^{\text {miss }}$ arises from mismeasurement of the jet energies. Data samples of photon events are used to predict this DY + jets background.

The final category comes from events with prompt neutrinos in addition to an OCSF pair from a $\mathrm{Z} / \gamma^{*}$ boson. This includes $\mathrm{WZ}$ and $\mathrm{ZZ}$ production and processes with lower cross section such as $\mathrm{t} \overline{\mathrm{Z}} \mathrm{Z}$ among others. These backgrounds are referred to as " $\mathrm{Z}+\nu$ " and can be important in the high- $p_{\mathrm{T}}^{\text {miss }}$ signal bins.

\subsection{Flavor-symmetric backgrounds}

The method of estimating the FS backgrounds relies on the fact that, for such processes, SF and DF events are produced at the same rate. This allows for prediction of the background yields in the SF sample from those in the DF sample by application of an appropriate correction factor, which is estimated from CRs in data. This factor corrects for different flavor-dependent reconstruction and identification efficiencies and for flavor-dependent trigger efficiencies, which can be different for electrons and muons.

For cases where the DF contribution is of sufficient statistical power to make an accurate prediction in the SF channel, a background estimate in the SF channel can therefore be obtained by applying a multiplicative correction factor, $R_{\mathrm{SF} / \mathrm{DF}}$, to the DF channel yield. The correction is determined in two independent ways, both based purely on control samples in the data. The two results are then combined using the weighted average according to their uncertainties to obtain the final factor. The first approach uses a direct 
measurement of this correction factor in a data CR independent of the baseline SR, and the second method involves a factorized approach of measuring the effects of reconstruction, identification, and trigger efficiencies separately and then combining them assuming the overall efficiency equal to the product of the individual components.

The direct measurement is performed in a CR requiring exactly two jets and $100<$ $p_{\mathrm{T}}^{\text {miss }}<150 \mathrm{GeV}$, excluding the dilepton invariant mass range $70<m_{\ell \ell}<110 \mathrm{GeV}$ to reduce contributions from $\mathrm{DY}+$ jets backgrounds. Here, $R_{\mathrm{SF} / \mathrm{DF}}$ is computed using the observed yield of SF and DF events, $R_{\mathrm{SF} / \mathrm{DF}}=\mathrm{N}_{\mathrm{SF}} / \mathrm{N}_{\mathrm{DF}}$. Data and simulation agree within $2 \%$ in this region. In simulation we find that $R_{\mathrm{SF} / \mathrm{DF}}$ differs by $1 \%$ when computed in the SR instead of the CR. We check the dependence of $R_{\mathrm{SF} / \mathrm{DF}}$ on the main kinematic variables used for the analysis in both data and simulation. Since the statistical power in data is limited, a systematic uncertainty of $4 \%$ is assigned based on the variations observed in simulation. The measured value of $R_{\mathrm{SF} / \mathrm{DF}}$ is $1.107 \pm 0.046$.

For the factorized approach, the ratio of muon to electron reconstruction and identification efficiencies, $r_{\mu / \mathrm{e}}$, is measured in a DY+jets-enriched $\mathrm{CR}$ requiring at least two jets, $p_{\mathrm{T}}^{\text {miss }}<50 \mathrm{GeV}$, and $60<m_{\ell \ell}<120 \mathrm{GeV}$. This results in a large sample of $\mathrm{e}^{ \pm} \mathrm{e}^{\mp}$ and $\mu^{ \pm} \mu^{\mp}$ events with similar kinematic distributions to those of the SR. Assuming the factorization of lepton efficiencies in an event, the efficiency ratio is measured as $r_{\mu / \mathrm{e}}=\sqrt{N_{\mu^{+} \mu^{-}} / N_{\mathrm{e}^{+} \mathrm{e}^{-}}}$. This ratio depends on the lepton $p_{\mathrm{T}}$ due to trigger and reconstruction efficiency differences, especially at low lepton $p_{\mathrm{T}}$. A parameterization as a function of the $p_{\mathrm{T}}$ of the less energetic lepton is used, and the functional form below is found to empirically describe the data:

$$
r_{\mu / \mathrm{e}}=C+\frac{\alpha}{p_{\mathrm{T}}} .
$$

Here $C$ and $\alpha$ are constants that are determined from a fit to data and checked using simulation. These fit parameters are determined to be $C=1.140 \pm 0.005$ and $\alpha=5.20 \pm$ $0.16 \mathrm{GeV}$. In addition to the fit uncertainty, a $10 \%$ systematic uncertainty is assigned to account for remaining variations observed when studying the dependence of $r_{\mu / \mathrm{e}}$ on the $p_{\mathrm{T}}$ of the more energetic lepton, $p_{\mathrm{T}}^{\text {miss }}$, and the jet multiplicity.

The trigger efficiencies for the three flavor combinations are used to define the factor $R_{\mathrm{T}}=\sqrt{\epsilon_{\mu^{ \pm} \mu^{\mp}}^{\mathrm{T}} \epsilon_{\mathrm{e}^{ \pm} \mathrm{e}^{\mp}}^{\mathrm{T}} / \epsilon_{\mathrm{e}^{ \pm} \mu^{\mp}}^{\mathrm{T}}}$, which takes into account the difference between SF and DF channels. The efficiencies are estimated from a control sample of events collected with a set of nonoverlapping triggers and range between $90-96 \%$, yielding a final value of $R_{\mathrm{T}}=$ $1.052 \pm 0.043$.

The final correction is $R_{\mathrm{SF} / \mathrm{DF}}=(1 / 2)\left(r_{\mu / \mathrm{e}}+r_{\mu / \mathrm{e}}^{-1}\right) R_{\mathrm{T}}$. The correction relies on the assumption that the number of produced DF events is twice the number of produced events in each SF sample. Thus, the number of observed DF events needs to be multiplied by $0.5 r_{\mu / \mathrm{e}} R_{\mathrm{T}}$ and $0.5 r_{\mu / \mathrm{e}}^{-1} R_{\mathrm{T}}$ to predict the number of dimuon and dielectron from FS processes, respectively. Summing $r_{\mu / \mathrm{e}}$ with its inverse leads to a large reduction in the associated uncertainty. Since $r_{\mu / \mathrm{e}}$ depends on the lepton kinematic variables, this correction is performed on an event-by-event basis. A separate correction is determined for each SR and combined with the correction from the direct measurement using the weighted average.

In the method described above, the statistical uncertainty in the predicted number of events is driven by the statistical uncertainty in the number of data events in the DF 
CR. Since $R_{\mathrm{SF} / \mathrm{DF}}$ is approximately one, the CR yield will be comparable to that of the FS background in the corresponding SR. In the on-Z SRs, the FS background is significantly reduced by the requirement that $m_{\ell \ell}$ lies within $5 \mathrm{GeV}$ of the $\mathrm{Z}$ boson mass. The expected FS background yields in the SRs are often of the order of a few events or less. We therefore modify the prediction method to obtain greater statistical power by relaxing the requirement on $m_{\ell \ell}$ for DF events, thereby increasing the number of events in the DF CR. An additional multiplicative factor, $\kappa$, is calculated and multiplied together with $R_{\mathrm{SF} / \mathrm{DF}}$ in order to translate this into a prediction for the SF SR. The factor $\kappa$ is defined as the number of DF events with $\left|m_{\mathrm{Z}}-m_{\ell \ell}\right|<5 \mathrm{GeV}$ divided by the number of DF events with $m_{\ell \ell}>20 \mathrm{GeV}$. It is determined from an DF control sample in simulation and validated in the DF data CRs. A value of $\kappa=0.065$ is measured from simulation. A systematic uncertainty of $30 \%$ is assigned by computing $\kappa$ in simulation for both the various on-Z SRs and bins of $p_{\mathrm{T}}^{\text {miss }}$. The largest observed difference from the nominal $\kappa$ value is taken as the systematic uncertainty. The value of $\kappa$ derived in data agrees with the result derived from simulation within the assigned uncertainty, and the statistical uncertainty in the derivation of $\kappa$ is negligible in comparison with the systematic uncertainty.

\subsection{Drell-Yan+jets backgrounds}

The $p_{\mathrm{T}}^{\text {miss }}$ from the DY+jets background is estimated from a sample of photon events in data using the $p_{\mathrm{T}}^{\text {miss }}$ "templates" method [13-16]. The main premise of this method is that $p_{\mathrm{T}}^{\text {miss }}$ in DY+jets events originates from the limited detector resolution when measuring the objects making up the hadronic system that recoils against the $\mathrm{Z}$ boson. The shape of the $p_{\mathrm{T}}^{\text {miss }}$ distribution can be estimated from a control sample of $\gamma+$ jets events where the jet system recoils against a photon instead of a $\mathrm{Z}$ boson. In addition to capturing the same resolution effects present in DY + jets events, the $\gamma+$ jets sample contains more events because of the branching fraction of $\mathrm{Z} \rightarrow \ell^{+} \ell^{-}$, and it does not have any contamination from signal events in the models considered. For SRs requiring at least one b-tagged jet, some of the observed $p_{\mathrm{T}}^{\text {miss }}$ can originate from neutrinos in semileptonic b-quark decays. To account for this effect, the $p_{\mathrm{T}}^{\text {miss }}$ templates are extracted from a control sample of $\gamma+$ jets events with the same b-tagging requirements as in each SR.

The $\gamma+$ jets events in data are selected with a suite of single-photon triggers with $p_{\mathrm{T}}$ thresholds varying from 22 to $165 \mathrm{GeV}$. The triggers with thresholds below $165 \mathrm{GeV}$ are prescaled such that only a fraction of accepted events are recorded, and the events are weighted by the trigger prescales to match the integrated luminosity collected with the signal dilepton triggers. In order to account for kinematic differences between the hadronic systems in the $\gamma+$ jets and the DY+jets samples, the $\gamma+$ jets sample is reweighted such that the photon $p_{\mathrm{T}}$ distribution matches the $\mathrm{Z} p_{\mathrm{T}}$ distribution in the $\mathrm{DY}+$ jets sample. $\mathrm{A}$ separate photon CR is defined for each of the on-Z SRs in table 1, where the same kinematic requirements are applied to the $\gamma+$ jets samples as in each SR. The reweighting in boson $p_{\mathrm{T}}$ is performed for each SR. Contributions to the photon sample from other SM processes with genuine $p_{\mathrm{T}}^{\text {miss }}$ from prompt neutrinos are subtracted as described below. The resulting $p_{\mathrm{T}}^{\text {miss }}$ distribution in each SR is then normalized to the observed dilepton data yield in the 
range $50<p_{\mathrm{T}}^{\text {miss }}<100 \mathrm{GeV}$, where $\mathrm{DY}+$ jets is the dominant background, after subtracting other background components.

The variable $M_{\mathrm{T} 2}$ used in the SR requires two visible objects as input and thus cannot be calculated in the same way in the photon sample. Instead, we emulate this requirement in $\gamma+$ jets by simulating the decay of the photon to two leptons. The decay is performed assuming the mother particle has the mass of a $\mathrm{Z}$ boson and the momentum of the photon reconstructed from data. We first consider a system of reference in which the mother particle is at rest. The decay to the leptons is performed in this system accounting for the angular dependence of spin correlations in the matrix element. Then a Lorentz transformation is applied to the emulated dilepton system in order to match the original momentum of the photon. The analysis requirements on $p_{\mathrm{T}}$ and $\eta$ for leptons are applied to the simulated decay products. The variable $M_{\mathrm{T} 2}(\ell \ell)$ is constructed using these leptons, showing good agreement with the distribution of $M_{\mathrm{T} 2}(\ell \ell)$ in genuine $\mathrm{DY}+$ jets events, and a selection is applied to this variable matching each SR requirement.

After selecting events with a high- $p_{\mathrm{T}}$ photon and large $p_{\mathrm{T}}^{\text {miss }}$, events from EW processes with genuine $p_{\mathrm{T}}^{\text {miss }}$, e.g. $\mathrm{W} \gamma$ where the $\mathrm{W}$ boson decays to $\ell \nu$, can be present in the tail of the $p_{\mathrm{T}}^{\text {miss }}$ distribution. To reduce the contamination from these EW processes, events in the photon sample are removed if they contain a lepton fulfilling the veto selections for the on- $\mathrm{Z}$ regions described in section 5. We then subtract the residual EW contamination using simulation. The relative size of the subtraction grows with increasing $p_{\mathrm{T}}^{\text {miss }}$ to be as large as around $50 \%$ of the prediction or 1 predicted event in the highest $p_{\mathrm{T}}^{\text {miss }}$ bins.

To validate the modeling of the subtracted EW processes, we define a data CR by selecting events with exactly one muon and one photon, requiring $p_{\mathrm{T}}^{\text {miss }}>50 \mathrm{GeV}$ and the transverse mass $M_{\mathrm{T}}$ of the muon and $p_{\mathrm{T}}^{\text {miss }}$ to be greater than $30 \mathrm{GeV}$. The muon must satisfy $p_{\mathrm{T}}>25 \mathrm{GeV}$, and the events are selected using a trigger that requires at least one isolated muon with $p_{\mathrm{T}}>24 \mathrm{GeV}$. This region consists of about $50 \% \mathrm{~W} \gamma$ events with the remainder coming primarily from $\mathrm{t} \overline{\mathrm{t}} \gamma$ events. Agreement is observed between data and the prediction from simulation. Based on the level of agreement between data and simulation in the kinematic distributions of photon $p_{\mathrm{T}}$ and $p_{\mathrm{T}}^{\text {miss }}$, we assign a systematic uncertainty of $30 \%$ in the subtraction of these EW processes.

The systematic uncertainty in the prediction takes into account the statistical uncertainty in the $\gamma+$ jets sample in each bin of $p_{\mathrm{T}}^{\text {miss }}$, which is the dominant uncertainty in the highest $p_{\mathrm{T}}^{\text {miss }}$ bins. The statistical uncertainty in the normalization region of $50<p_{\mathrm{T}}^{\text {miss }}<$ $100 \mathrm{GeV}$ is included and ranges from $7-30 \%$. A closure test of the method is performed in simulation, using $\gamma+$ jets to predict the yield of DY +jets in each analysis bin. An uncertainty is assigned from the results of this test as the larger of the difference between the $\gamma+$ jets prediction and the DY + jets yield for each $p_{\mathrm{T}}^{\text {miss }}$ region or the simulation statistical uncertainty. The values vary between 10 and $80 \%$ depending on the $p_{\mathrm{T}}^{\text {miss }}$ region, with the larger values coming from regions with low statistics in simulation.

The template method is also used to provide a prediction for the background from DY+jets in the edge SRs, where this background is significantly smaller due to the $m_{\ell \ell}$ requirements. We define the ratio $r_{\text {out/in }}$ in a DY+jets-dominated sample as the number of SF events in a given bin of $m_{\ell \ell}$ divided by the SF yield within $86<m_{\ell \ell}<96 \mathrm{GeV}$. The 
ratio is measured in a DY+jets-dominated $\mathrm{CR}$ requiring at least two jets, $p_{\mathrm{T}}^{\text {miss }}<50 \mathrm{GeV}$, and $M_{\mathrm{T} 2}(\ell \ell)>80 \mathrm{GeV}$. Different-flavor yields in both the numerator and denominator are subtracted from the respective SF yields in order to correct for small FS contributions in the region where $r_{\text {out/in }}$ is measured. The value of $r_{\text {out/in }}$ ranges from 0.001 to 0.16 for the different bins in $m_{\ell \ell}$. The DY+jets background contribution to each $m_{\ell \ell}$ bin is computed by multiplying the on-Z prediction by $r_{\text {out/in }}$. The dependence of $r_{\text {out/in }}$ on $p_{\mathrm{T}}^{\text {miss }}$ and the jet multiplicity are studied in the data CR. Based on the statistical precision of this check, and the observed variations as a function of these variables, we assign an uncertainty of 50 (100)\% to $r_{\text {out } / \text { in }}$ in the $m_{\ell \ell}$ bins below (above) $150 \mathrm{GeV}$.

\subsection{Backgrounds with $\mathrm{Z}$ bosons plus genuine $p_{\mathrm{T}}^{\text {miss }}$}

The $p_{\mathrm{T}}^{\text {miss }}$ template method only predicts instrumental $p_{\mathrm{T}}^{\text {miss }}$ from jet mismeasurement and thus does not include the genuine $p_{\mathrm{T}}^{\text {miss }}$ from prompt neutrinos expected in processes like $\mathrm{W}(\ell \nu) \mathrm{Z}(\ell \ell), \mathrm{Z}(\ell \ell) \mathrm{Z}(\nu \nu)$, or lower cross section processes such as t⿱亠tZZ. These processes can be a substantial fraction of the background at high $p_{\mathrm{T}}^{\mathrm{miss}}$ and are estimated using simulation.

The prediction from simulation is validated by comparing to data in CRs requiring three or four leptons. A region enriched in WZ events is selected by requiring exactly three leptons, at least two jets, no b-tagged jets, $p_{\mathrm{T}}^{\text {miss }}>60 \mathrm{GeV}$, and an OCSF lepton pair with

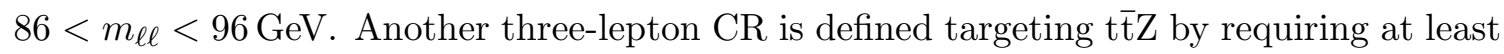
two jets, at least two b-tagged jets, $p_{\mathrm{T}}^{\text {miss }}>30 \mathrm{GeV}$, and an OCSF lepton pair as in the WZ region. A four-lepton $\mathrm{CR}$ targeting $\mathrm{ZZ}$ is constructed by requiring four leptons with two OCSF pairs satisfying $m_{\ell \ell}>20 \mathrm{GeV}$, to remove low-mass resonances, and at least two jets.

After subtracting the other processes using simulation in each region, simulation-todata scale factors of $0.98 \pm 0.11,1.58 \pm 0.49$, and $1.31 \pm 0.29$ are observed for WZ, ZZ, and $t \overline{t Z}$ backgrounds respectively. We use the scale factor values to correct the prediction from simulation for each process. Based on the statistical uncertainty in these CRs, and the agreement between data and simulation in distributions of kinematic variables such as $p_{\mathrm{T}}^{\text {miss }}$ and the number of jets, we assign systematic uncertainties of $30 \%$ for the WZ and t⿱亠幺Z background predictions and $50 \%$ for the $\mathrm{ZZ}$ prediction. As all other effects are subdominant, we do not assign further uncertainties to these backgrounds.

\section{Kinematic fit}

A simultaneous extended unbinned maximum likelihood fit is performed in the $m_{\ell \ell}$ distributions of $\mathrm{e}^{+} \mathrm{e}^{-}, \mu^{+} \mu^{-}$, and $\mathrm{e}^{ \pm} \mu^{\mp}$ events to search for a kinematic edge. The fit is performed after the kinematic selection labeled "Edge fit" in table 1. The likelihood model contains three components: an FS background component, a DY+jets background component, and a signal component. The $\mathrm{Z}+\nu$ background is contained within the DY+jets component in this method, as both have the same $m_{\ell \ell}$ shape.

The FS background component is described using a CB function $\mathcal{P}_{\mathrm{CB}}\left(m_{\ell \ell}\right)$ :

$$
\mathcal{P}_{\mathrm{CB}}\left(m_{\ell \ell}\right)= \begin{cases}\exp \left[-\frac{\left(m_{\ell \ell}-\mu_{\mathrm{CB}}\right)^{2}}{2 \Gamma_{\mathrm{CB}}^{2}}\right] & \text { if } \frac{m_{\ell \ell}-\mu_{\mathrm{CB}}}{\Gamma_{\mathrm{CB}}}<\alpha \\ A\left(B+\frac{m_{\ell \ell}-\mu_{\mathrm{CB}}}{\Gamma_{\mathrm{CB}}}\right)^{-n} & \text { if } \frac{m_{\ell \ell}-\mu_{\mathrm{CB}}}{\Gamma_{\mathrm{CB}}}>\alpha\end{cases}
$$


where

$$
A=\left(\frac{n}{|\alpha|}\right)^{n} \exp \left(-\frac{|\alpha|^{2}}{2}\right) \quad \text { and } \quad B=\frac{n}{|\alpha|}-|\alpha| .
$$

The FS background model has five free parameters: the overall normalization, the mean $\mu_{\mathrm{CB}}$ and width $\Gamma_{\mathrm{CB}}$ of the Gaussian part, the transition point $\alpha$ between the Gaussian part and the power law tail, and the power law parameter $n$.

The DY+jets background component is modeled with the sum of an exponential function, which describes the low-mass rise, and a Breit-Wigner function with a mean and width set to the nominal $\mathrm{Z}$ boson values [60], which accounts for the $\mathrm{Z}$ boson lineshape. To account for the experimental resolution, the Breit-Wigner function is convolved with a double-sided CB function

$$
\mathcal{P}_{\mathrm{DSCB}}\left(m_{\ell \ell}\right)= \begin{cases}A_{1}\left(B_{1}-\frac{m_{\ell \ell}-\mu_{\mathrm{DSCB}}}{\Gamma_{\mathrm{DSCB}}}\right)^{-n_{1}} & \text { if } \frac{m_{\ell \ell}-\mu_{\mathrm{DSCB}}}{\Gamma_{\mathrm{DSCB}}}<-\alpha_{1} \\ \exp \left[-\frac{\left(m_{\ell \ell}-\mu_{\mathrm{DSCB}}\right)^{2}}{2 \Gamma_{\mathrm{DSCB}}^{2}}\right] & \text { if }-\alpha_{1}<\frac{m_{\ell \ell}-\mu_{\mathrm{DSCB}}}{\Gamma_{\mathrm{DSCB}}}<\alpha_{2} \\ A_{2}\left(B_{2}+\frac{m_{\ell \ell}-\mu_{\mathrm{DSCB}}}{\Gamma_{\mathrm{DSCB}}}\right)^{-n_{2}} & \text { if } \frac{m_{\ell \ell}-\mu_{\mathrm{DSCB}}}{\Gamma_{\mathrm{DSCB}}}>\alpha_{2}\end{cases}
$$

where $\mu_{\mathrm{DSCB}}$ and $\Gamma_{\mathrm{DSCB}}$ are the mean and width, respectively, of the $\mathrm{CB}$ function, and $\alpha_{1}$ and $\alpha_{2}$ are the transition points. The full model for the on-Z DY+jets background line shape is thus

$$
\mathcal{P}_{\mathrm{DY}, \text { on-Z }}\left(m_{\ell \ell}\right)=\int \mathcal{P}_{\mathrm{DSCB}}\left(m_{\ell \ell}\right) \mathcal{P}_{\mathrm{BW}}\left(m_{\ell \ell}-m^{\prime}\right) \mathrm{d} m^{\prime},
$$

where $\mathcal{P}_{\mathrm{BW}}$ is the Breit-Wigner function. The complete DY+jets background model has nine free parameters.

The signal component is described by a triangular shape, convolved with a Gaussian distribution to account for the experimental resolution:

$$
\mathcal{P}_{\mathrm{S}}\left(m_{\ell \ell}\right) \propto \frac{1}{\sqrt{2 \pi} \Gamma_{\ell \ell}} \int_{0}^{m_{\ell \ell}^{\text {edge }}} y \exp \left[-\frac{\left(m_{\ell \ell}-y\right)^{2}}{2 \Gamma_{\ell \ell}^{2}}\right] \mathrm{d} y .
$$

The signal model has two free parameters: the fitted signal yield and the position of the edge, $m_{\ell \ell}^{\text {edge }}$.

As the first step, a fit is performed separately for $\mathrm{e}^{+} \mathrm{e}^{-}$and $\mu^{+} \mu^{-}$events in a DY+jetsenriched $\mathrm{CR}$ requiring at least two jets and $p_{\mathrm{T}}^{\text {miss }}<50 \mathrm{GeV}$, to determine the shape of backgrounds containing a $\mathrm{Z}$ boson. The parameters of the DY+jets background shape are then fixed and only the normalizations of these backgrounds are free parameters in the subsequent fit. The final fit is performed simultaneously to the dilepton invariant mass distributions in the $\mathrm{e}^{+} \mathrm{e}^{-}, \mu^{+} \mu^{-}$, and $\mathrm{e}^{ \pm} \mu^{\mp}$ samples. The model for the FS background is the same for the SF and DF events. The $R_{\mathrm{SF} / \mathrm{DF}}$ factor is treated as a nuisance parameter, parameterized by a Gaussian distribution with a mean value and standard deviation given by the value of $R_{\mathrm{SF} / \mathrm{DF}}$ and its uncertainties (see section 6.1 ). The final fit has ten free parameters: a normalization for each of the three fit components, four parameters for the shape of the FS background, $R_{\mathrm{SF} / \mathrm{DF}}$, the relative fraction of dielectron and dimuon events in the FS prediction, and the position of the signal edge. 


\section{Results}

The observed number of events in the SRs are compared with the background estimates for the on-Z strong- and EW-production and the edge searches. The covariance and correlation matrices of the background predictions in the different SRs are also provided in appendix A to facilitate reinterpretation of these results. For the edge search, the fit is performed to search for a kinematic edge in the $m_{\ell \ell}$ spectrum.

\subsection{Results of the search in the on- $Z$ signal regions}

The results for the SRs of the on-Z strong-production search are presented in table 2 . The corresponding $p_{\mathrm{T}}^{\mathrm{miss}}$ distributions are shown in figure 3 . No significant deviations are observed with respect to SM expectations.

The results for the EW SRs in the on-Z search are shown in table 3. The corresponding $p_{\mathrm{T}}^{\text {miss }}$ distributions are shown in figure 4 . The observed data are also consistent with the background prediction.

\subsection{Results of the edge search}

The edge search features seven distinct $m_{\ell \ell}$ regions, each of which is divided into two bins using the likelihood discriminant, resulting in fourteen SRs. In addition, two aggregate regions integrating the SRs below and above the $\mathrm{Z}$ boson mass have been considered in

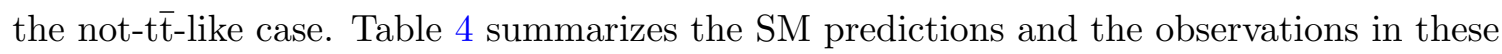
SRs. A graphical representation of these results is shown in figure 5, including the relative contributions of the different backgrounds.

At high mass and in the not-t $\overline{\mathrm{t}}$-like regions, the uncertainty in the background prediction is driven by the statistical uncertainty in the number of events in the DF control sample. There is good agreement between prediction and observation for all SRs. The largest deviation is observed in the not-t $\overline{\mathrm{t}}$-like region for masses between 96 and $150 \mathrm{GeV}$, with an excess corresponding to a local significance of 2.0 standard deviations.

The dilepton mass distributions and the results of the kinematic fit are shown in figure 6. Table 5 presents a summary of the fit results. A signal yield of $61 \pm 28$ events is obtained when evaluating the signal hypothesis in the baseline SR, with a fitted edge position of $144.2_{-2.2}^{+3.3} \mathrm{GeV}$. This is in agreement with the upwards fluctuations in the mass region between 96 and $150 \mathrm{GeV}$ in the counting experiment and corresponds to a local significance of 2.3 standard deviations. To estimate the global $p$-value [61] of the result, the test statistic $-2 \ln Q$, where $Q$ denotes the ratio of the fitted likelihood value for the signal-plus-background hypothesis to the background-only hypothesis, is evaluated on data and compared to the respective quantity on a large sample of background-only pseudo-experiments where the edge position can have any value. The resulting $p$-value is interpreted as the one-sided tail probability of a Gaussian distribution and corresponds to an excess in the observed number of events compared to the SM background prediction with a global significance of 1.5 standard deviations. 


\begin{tabular}{|c|c|c|c|c|}
\hline \multirow[t]{6}{*}{ SRA, b veto } & $p_{\mathrm{T}}^{\text {miss }}[\mathrm{GeV}]$ & $100-150$ & $150-250$ & $>250$ \\
\hline & $\mathrm{DY}+\mathrm{jets}$ & $13.6 \pm 3.1$ & $2.5 \pm 0.9$ & $3.3 \pm 2.4$ \\
\hline & $\mathrm{FS}$ & $0.4_{-0.2}^{+0.3}$ & $0.2_{-0.1}^{+0.2}$ & $0.2_{-0.1}^{+0.2}$ \\
\hline & $\mathrm{Z}+\nu$ & $0.8 \pm 0.3$ & $1.4 \pm 0.4$ & $2.4 \pm 0.8$ \\
\hline & Total background & $14.8 \pm 3.2$ & $4.0 \pm 1.0$ & $5.9 \pm 2.5$ \\
\hline & Data & 23 & 5 & 4 \\
\hline \multirow[t]{6}{*}{ SRA, b tag } & $p_{\mathrm{T}}^{\text {miss }}[\mathrm{GeV}]$ & $100-150$ & $150-250$ & $>250$ \\
\hline & $\mathrm{DY}+$ jets & $8.2 \pm 2.1$ & $1.2 \pm 0.5$ & $0.5 \pm 0.3$ \\
\hline & FS & $2.3 \pm 0.8$ & $1.7_{-0.6}^{+0.7}$ & $0.1_{-0.1}^{+0.2}$ \\
\hline & $\mathrm{Z}+\nu$ & $1.9 \pm 0.4$ & $2.0 \pm 0.5$ & $1.8 \pm 0.6$ \\
\hline & Total background & $12.4 \pm 2.3$ & $4.9 \pm 1.0$ & $2.5 \pm 0.7$ \\
\hline & Data & 14 & 7 & 1 \\
\hline \multirow[t]{6}{*}{$\mathrm{SRB}, \mathrm{b}$ veto } & $p_{\mathrm{T}}^{\text {miss }}[\mathrm{GeV}]$ & $100-150$ & $150-250$ & $>250$ \\
\hline & $\mathrm{DY}+$ jets & $12.8 \pm 2.3$ & $0.9 \pm 0.3$ & $0.4 \pm 0.2$ \\
\hline & FS & $0.4_{-0.2}^{+0.3}$ & $0.4_{-0.2}^{+0.3}$ & $0.1_{-0.1}^{+0.2}$ \\
\hline & $\mathrm{Z}+\nu$ & $0.3 \pm 0.1$ & $0.7 \pm 0.2$ & $1.2 \pm 0.4$ \\
\hline & Total background & $13.6 \pm 2.4$ & $2.0 \pm 0.5$ & $1.6 \pm 0.4$ \\
\hline & Data & 10 & 4 & 0 \\
\hline \multirow[t]{6}{*}{ SRB, b tag } & $p_{\mathrm{T}}^{\text {miss }}[\mathrm{GeV}]$ & $100-150$ & $150-250$ & $>250$ \\
\hline & $\mathrm{DY}+$ jets & $7.7 \pm 3.2$ & $4.0 \pm 3.4$ & $0.1 \pm 0.1$ \\
\hline & FS & $1.4_{-0.5}^{+0.6}$ & $1.1_{-0.4}^{+0.5}$ & $0.2_{-0.1}^{+0.2}$ \\
\hline & $\mathrm{Z}+\nu$ & $2.0 \pm 0.5$ & $2.3 \pm 0.6$ & $1.0 \pm 0.3$ \\
\hline & Total background & $11.1 \pm 3.3$ & $7.4_{-3.4}^{+3.5}$ & $1.3_{-0.3}^{+0.4}$ \\
\hline & Data & 10 & 5 & 0 \\
\hline \multirow[t]{6}{*}{ SRC, b veto } & $p_{\mathrm{T}}^{\text {miss }}[\mathrm{GeV}]$ & $100-150$ & $>150$ & \\
\hline & DY+jets & $1.2 \pm 0.4$ & $0.1 \pm 0.1$ & \\
\hline & FS & $0.4_{-0.2}^{+0.3}$ & $0.1_{-0.1}^{+0.2}$ & \\
\hline & $\mathrm{Z}+\nu$ & $0.1 \pm 0.1$ & $0.5 \pm 0.2$ & \\
\hline & Total background & $1.7 \pm 0.5$ & $0.7_{-0.2}^{+0.3}$ & \\
\hline & Data & 4 & 0 & \\
\hline \multirow[t]{6}{*}{ SRC, b tag } & $p_{\mathrm{T}}^{\text {miss }}[\mathrm{GeV}]$ & $100-150$ & $>150$ & \\
\hline & DY+jets & $0.1 \pm 0.4$ & $0.0 \pm 0.3$ & \\
\hline & FS & $0.0_{-0.0}^{+0.1}$ & $0.3 \pm 0.2$ & \\
\hline & $\mathrm{Z}+\nu$ & $0.6 \pm 0.2$ & $0.6 \pm 0.2$ & \\
\hline & Total background & $0.8 \pm 0.5$ & $0.9_{-0.4}^{+0.5}$ & \\
\hline & Data & 2 & 2 & \\
\hline
\end{tabular}

Table 2. Predicted and observed event yields are shown for the on-Z strong-production SRs, for each $p_{\mathrm{T}}^{\text {miss }}$ bin defined in table 1 . The uncertainties shown include both statistical and systematic components. 

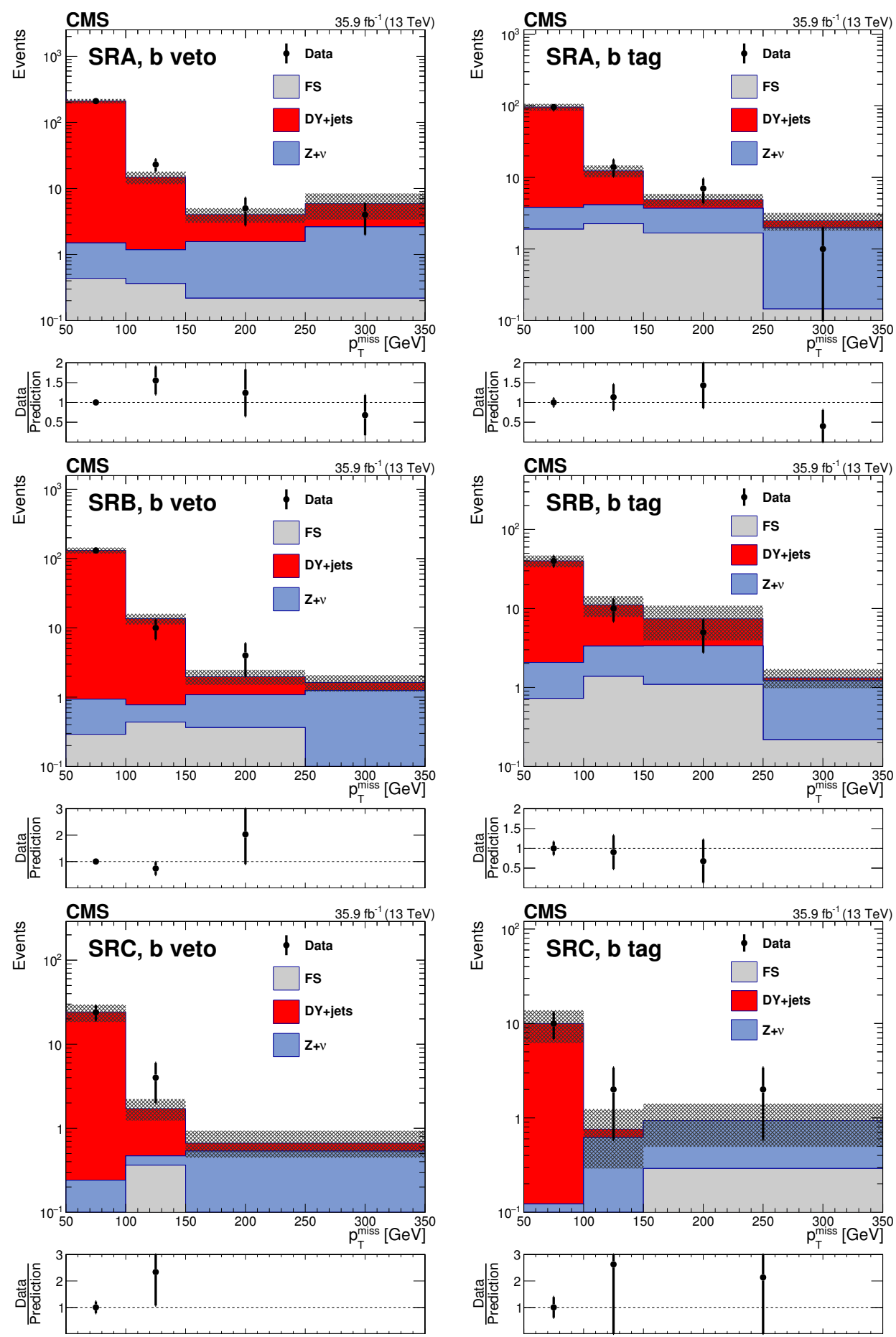

Figure 3. The $p_{\mathrm{T}}^{\text {miss }}$ distribution is shown for data compared to the background prediction in the on-Z strong-production SRs with no b-tagged jets (left) and at least $1 \mathrm{~b}$-tagged jet (right). The rows show SRA (upper), SRB (middle), and SRC (lower). The lower panel of each plot shows the ratio of observed data to the predicted value in each bin. The hashed band in the upper panels shows the total uncertainty in the background prediction, including statistical and systematic components. The $p_{\mathrm{T}}^{\text {miss }}$ template prediction for each SR is normalized to the first bin of each distribution, and therefore the prediction agrees with the data by construction. 

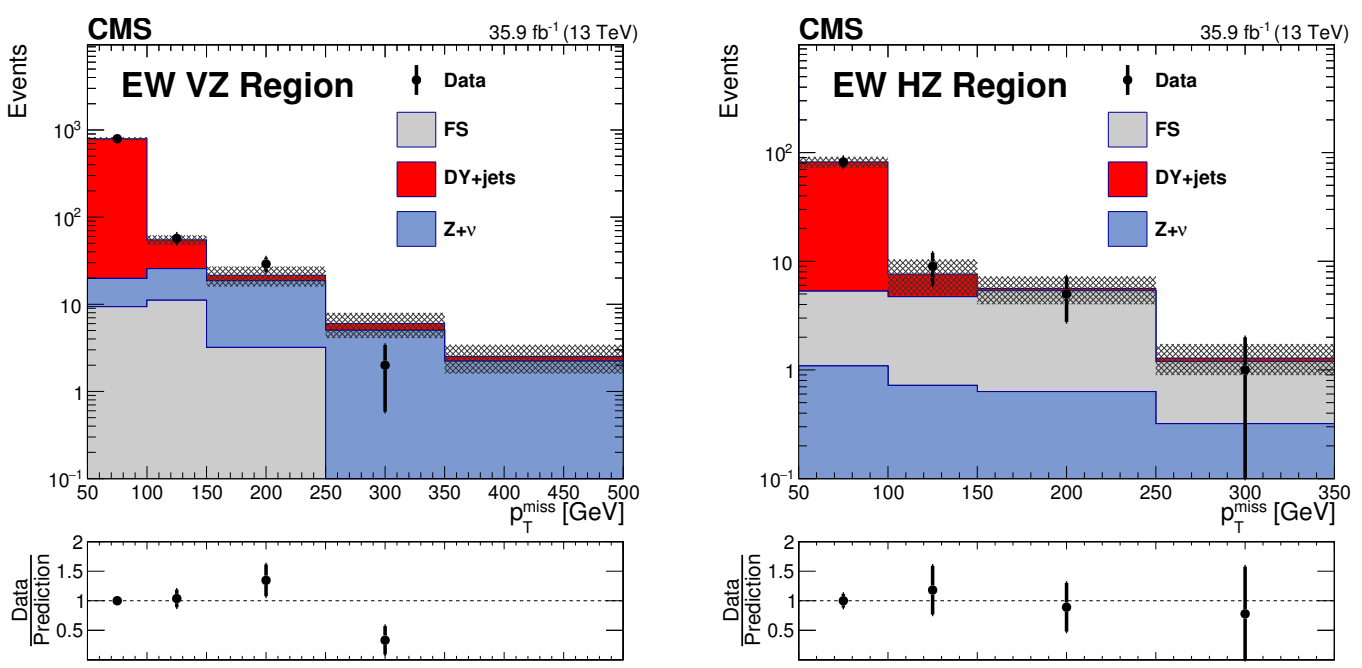

Figure 4. The $p_{\mathrm{T}}^{\mathrm{miss}}$ distribution is shown for data compared to the background prediction in the on-Z VZ (left) and HZ (right) electroweak-production SRs. The lower panel of each figure shows the ratio of observed data to the predicted value in each bin. The hashed band in the upper panels shows the total uncertainty in the background prediction, including statistical and systematic sources. The $p_{\mathrm{T}}^{\mathrm{miss}}$ template prediction for each SR is normalized to the first bin of each distribution, and therefore the prediction agrees with the data by construction.

\begin{tabular}{|c|c|c|c|c|c|}
\hline \multirow[t]{6}{*}{ VZ } & $p_{\mathrm{T}}^{\mathrm{miss}}[\mathrm{GeV}]$ & $100-150$ & $150-250$ & $250-350$ & $>350$ \\
\hline & $\mathrm{DY}+$ jets & $29.3 \pm 4.4$ & $2.9 \pm 2.0$ & $1.0 \pm 0.7$ & $0.3 \pm 0.3$ \\
\hline & FS & $11.1 \pm 3.6$ & $3.2 \pm 1.1$ & $0.1_{-0.1}^{+0.2}$ & $0.1_{-0.1}^{+0.2}$ \\
\hline & $\mathrm{Z}+\nu$ & $14.5 \pm 4.0$ & $15.5 \pm 5.1$ & $5.0 \pm 1.8$ & $2.2 \pm 0.9$ \\
\hline & Total background & $54.9 \pm 7.0$ & $21.6 \pm 5.6$ & $6.0 \pm 1.9$ & $2.5 \pm 0.9$ \\
\hline & Data & 57 & 29 & 2 & 0 \\
\hline \multirow[t]{6}{*}{$\mathrm{HZ}$} & $p_{\mathrm{T}}^{\operatorname{miss}}[\mathrm{GeV}]$ & $100-150$ & $150-250$ & $>250$ & \\
\hline & $\mathrm{DY}+$ jets & $2.9 \pm 2.4$ & $0.3 \pm 0.2$ & $0.1 \pm 0.1$ & \\
\hline & FS & $4.0 \pm 1.4$ & $4.7 \pm 1.6$ & $0.9 \pm 0.4$ & \\
\hline & $\mathrm{Z}+\nu$ & $0.7 \pm 0.2$ & $0.6 \pm 0.2$ & $0.3 \pm 0.1$ & \\
\hline & Total background & $7.6 \pm 2.8$ & $5.6 \pm 1.6$ & $1.3 \pm 0.4$ & \\
\hline & Data & 9 & 5 & 1 & \\
\hline
\end{tabular}

Table 3. Predicted and observed event yields are shown for the EW on-Z SRs, for each $p_{\mathrm{T}}^{\mathrm{miss}}$ bin defined in table 1 . The uncertainties shown include both statistical and systematic sources. 


\begin{tabular}{|c|c|c|c|c|c|}
\hline$m_{\ell \ell}$ range $[\mathrm{GeV}]$ & FS & $\mathrm{DY}+$ jets & $\mathrm{Z}+\nu$ & Total background & Data \\
\hline \multicolumn{6}{|c|}{$\mathrm{t} \overline{\mathrm{t}}$-like } \\
\hline $20-60$ & $291_{-20}^{+21}$ & $0.4 \pm 0.3$ & $1.4 \pm 0.5$ & $293_{-20}^{+21}$ & 273 \\
\hline $60-86$ & $181_{-15}^{+16}$ & $0.9 \pm 0.7$ & $8.8 \pm 3.4$ & $190_{-15}^{+16}$ & 190 \\
\hline $96-150$ & $176_{-14}^{+15}$ & $1.1 \pm 0.9$ & $6.0 \pm 2.4$ & $182_{-15}^{+16}$ & 192 \\
\hline $150-200$ & $73_{-9}^{+10}$ & $0.1 \pm 0.1$ & $0.4 \pm 0.2$ & $74_{-9}^{+10}$ & 66 \\
\hline $200-300$ & $46.9_{-7.3}^{+8.4}$ & $<0.1$ & $0.3 \pm 0.1$ & $47.3_{-7.3}^{+8.4}$ & 42 \\
\hline $300-400$ & $18.5_{-4.5}^{+5.7}$ & $<0.1$ & $<0.1$ & $18.6_{-4.5}^{+5.7}$ & 11 \\
\hline$>400$ & $4.3_{-2.1}^{+3.4}$ & $<0.1$ & $<0.1$ & $4.5_{-2.1}^{+3.4}$ & 4 \\
\hline \multicolumn{6}{|c|}{ 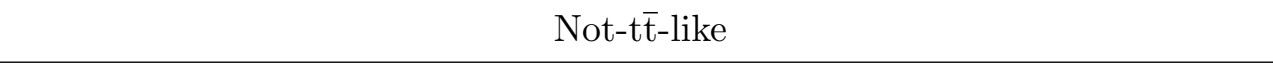 } \\
\hline $20-60$ & $3.3_{-1.8}^{+3.2}$ & $0.7 \pm 0.5$ & $1.4 \pm 0.5$ & $5.3_{-1.9}^{+3.3}$ & 6 \\
\hline $60-86$ & $3.3_{-1.8}^{+3.2}$ & $1.6 \pm 1.3$ & $6.9 \pm 2.7$ & $11.8_{-3.5}^{+4.4}$ & 19 \\
\hline $96-150$ & $6.6_{-2.6}^{+3.9}$ & $1.9 \pm 1.5$ & $6.8 \pm 2.7$ & $15.3_{-4.1}^{+5.0}$ & 28 \\
\hline $150-200$ & $5.5_{-2.4}^{+3.7}$ & $0.2 \pm 0.3$ & $0.7 \pm 0.3$ & $6.4_{-2.4}^{+3.7}$ & 7 \\
\hline $200-300$ & $3.3_{-1.8}^{+3.2}$ & $0.2 \pm 0.2$ & $0.5 \pm 0.2$ & $3.9_{-1.8}^{+3.2}$ & 4 \\
\hline $300-400$ & $3.3_{-1.8}^{+3.2}$ & $<0.1$ & $0.2 \pm 0.1$ & $3.5_{-1.8}^{+3.2}$ & 0 \\
\hline$>400$ & $1.1_{-0.9}^{+2.5}$ & $<0.1$ & $0.4 \pm 0.2$ & $1.6_{-0.9}^{+2.5}$ & 5 \\
\hline \multicolumn{6}{|c|}{ 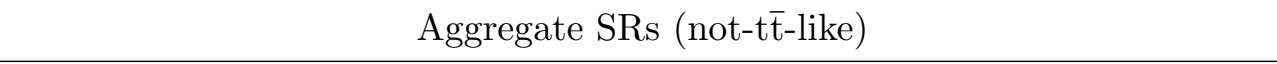 } \\
\hline $20-86$ & $6.5_{-2.6}^{+3.9}$ & $2.3 \pm 1.5$ & $8.3 \pm 3.2$ & $17.1_{-4.4}^{+5.3}$ & 25 \\
\hline$>96$ & $19.6_{-4.6}^{+5.8}$ & $2.4 \pm 1.6$ & $8.5 \pm 3.4$ & $30.6_{-6.0}^{+7.0}$ & 44 \\
\hline
\end{tabular}

Table 4. Predicted and observed yields in each bin of the edge search counting experiment. The uncertainties shown include both statistical and systematic sources.

\begin{tabular}{|lc|}
\hline DY+jets yield & $191 \pm 19$ \\
FS yield & $768 \pm 24$ \\
$R_{\mathrm{SF} / \mathrm{DF}}$ & $1.07 \pm 0.03$ \\
Signal yield & $61 \pm 28$ \\
$m_{\ell \ell}^{\text {edge }}$ & $144.2_{-2.2}^{+3.3} \mathrm{GeV}$ \\
\hline Local significance & 2.3 s.d. \\
Global significance & 1.5 s.d. \\
\hline
\end{tabular}

Table 5. Results of the unbinned maximum likelihood fit for event yields in the edge fit SR of table 1, including the DY+jets and FS background components, along with the fitted signal contribution and edge position. The fitted value for $R_{\mathrm{SF} / \mathrm{DF}}$ and the local and global signal significances in terms of standard deviations are also given. The uncertainties account for both statistical and systematic components. 


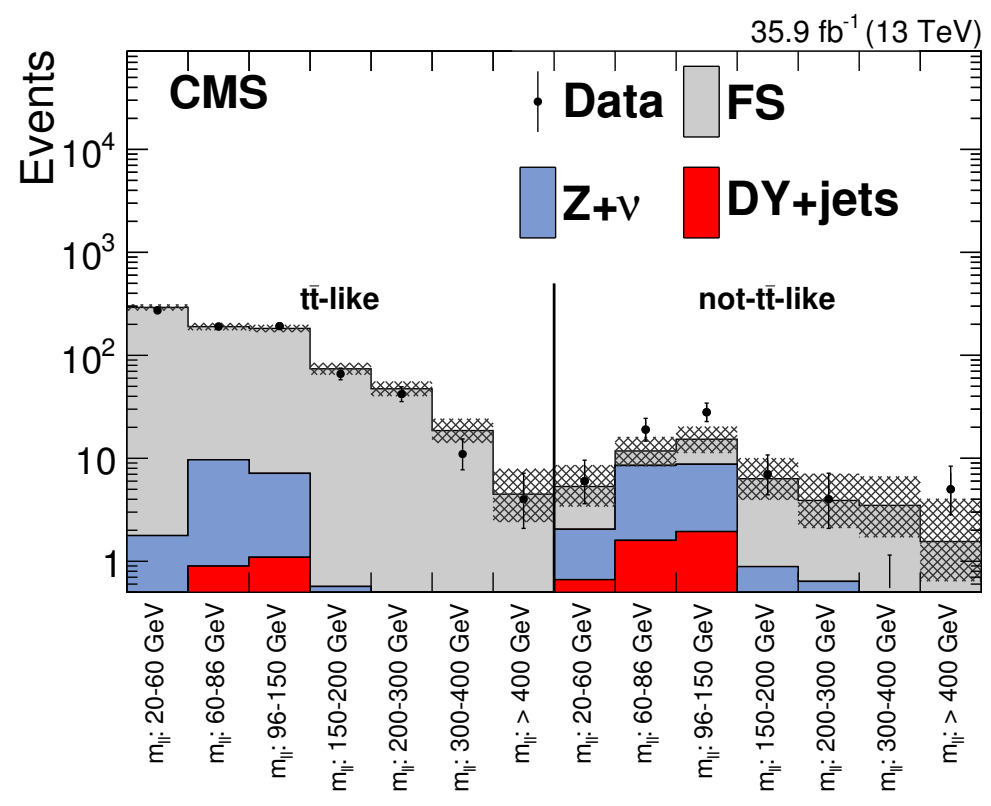

Figure 5. Results of the counting experiment of the edge search. For each SR, the number of observed events, shown as black data points, is compared to the total background estimate. The hashed band shows the total uncertainty in the background prediction, including statistical and systematic sources.
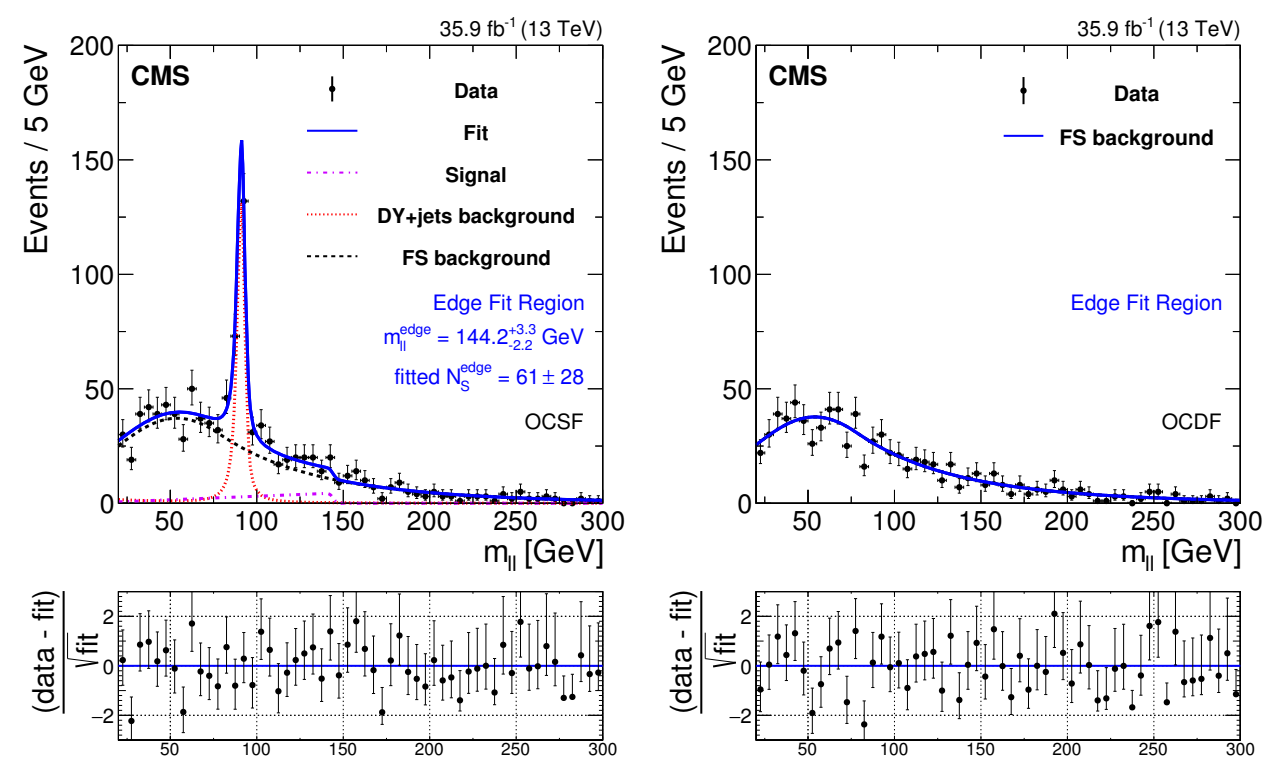

Figure 6. Fit of the dilepton mass distributions to the signal-plus-background hypothesis in the "Edge fit" SR from table 1, projected on the same-flavor (left) and different-flavor (right) event samples. The fit shape is shown as a solid blue line. The individual fit components are indicated by dashed and dotted lines. The FS background is shown with a black dashed line. The DY+jets background is displayed with a red dotted line. The extracted signal component is displayed with a purple dash-dotted line. The lower panel in each plot shows the difference between the observation and the fit, divided by the square root of the number of fitted events. 


\begin{tabular}{|lc|}
\hline Source of uncertainty & Uncertainty $(\%)$ \\
\hline Integrated luminosity & 2.5 \\
Lepton reconstruction and isolation & 5 \\
Fast simulation lepton efficiency & 4 \\
b tag modeling & $0-5$ \\
Trigger modeling & 3 \\
Jet energy scale & $0-5$ \\
ISR modeling & $0-2.5$ \\
Pileup & $1-2$ \\
Fast simulation $p_{\mathrm{T}}^{\text {miss }}$ modeling & $0-4$ \\
Renorm./fact. scales & $1-3$ \\
Statistical uncertainty & $1-15$ \\
\hline Total uncertainty & $9-18$ \\
\hline
\end{tabular}

Table 6. Systematic uncertainties taken into account for the signal yields and their typical values.

\section{Interpretation}

The results are interpreted in terms of the simplified models defined in section 2. Upper limits on the cross section (assuming branching fractions presented in section 2) have been calculated at $95 \%$ confidence level (CL) using the $\mathrm{CL}_{\mathrm{S}}$ criterion and an asymptotic formulation [62-65], taking into account the statistical and systematic uncertainties in the signal yields and the background predictions.

\subsection{Systematic uncertainty in the signal yield}

The systematic uncertainties in the signal yield are summarized in table 6 . The uncertainty in the measurement of the integrated luminosity is $2.5 \%$ [66]. The uncertainty in the lepton identification and isolation efficiency amounts to $5 \%$ in the signal acceptance. A further uncertainty of $4 \%$ arises from the modeling of the lepton efficiency in the fast simulation used for signal. The uncertainties in the $\mathrm{b}$ tagging efficiency and mistag probability are between 0 and $5 \%$ depending on the signal model and masses probed. The uncertainty in the trigger efficiency is $3 \%$. The uncertainty in the jet energy scale varies between $0-$ $5 \%$ depending on the signal kinematics. The uncertainty associated with the modeling of initial-state radiation (ISR) is $0-2.5 \%$. Determining the signal acceptance in high- and low-pileup regimes separately yields an uncertainty of $1-2 \%$. The uncertainty in the $p_{\mathrm{T}}^{\text {miss }}$ modeling in fast simulation amounts to $0-4 \%$. Generator renormalization and factorization scales are varied up and down by a factor of two, resulting in an uncertainty in the signal acceptance of $1-3 \%$. Finally the statistical uncertainty in the number of simulated events is also considered and found to be in the range 1-15\%, depending on the SR and mass point. 


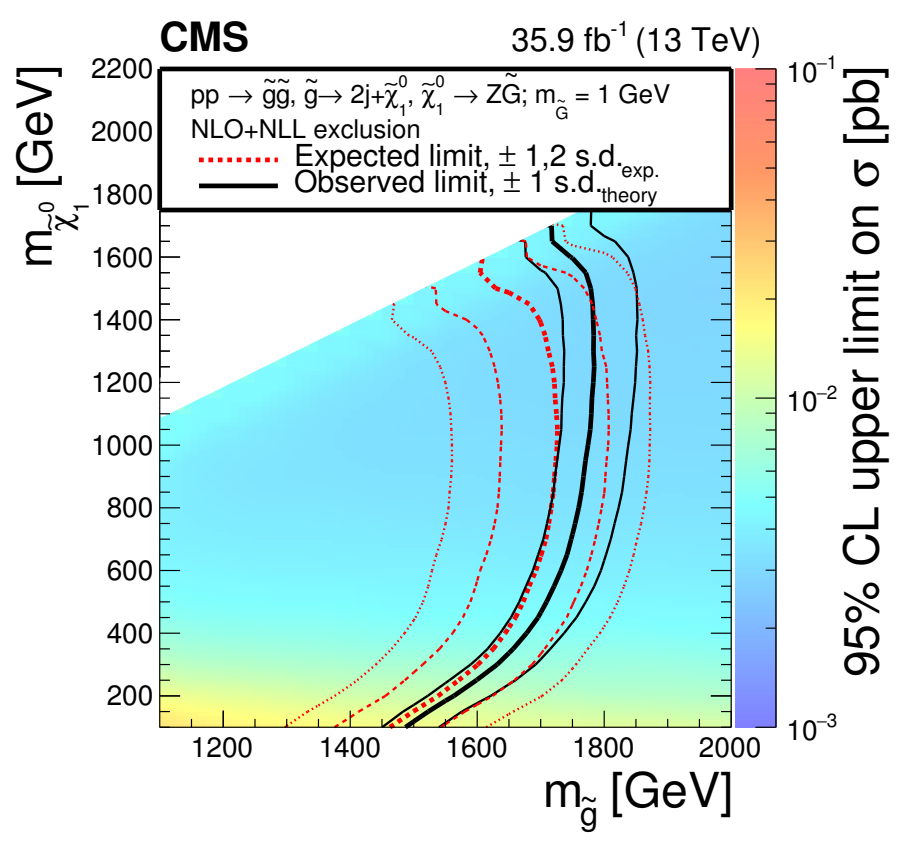

Figure 7. Cross section upper limit and exclusion contours at 95\% CL for the gluino GMSB model as a function of the $\widetilde{\mathrm{g}}$ and $\widetilde{\chi}_{1}^{0}$ masses, obtained from the results of the strong production on-Z search. The region to the left of the thick red dotted (black solid) line is excluded by the expected (observed) limit. The thin red dotted curves indicate the regions containing 68 and $95 \%$ of the distribution of limits expected under the background-only hypothesis. The thin solid black curves show the change in the observed limit due to variation of the signal cross sections within their theoretical uncertainties.

\subsection{Interpretations using simplified models}

The gluino GMSB model leads to a signature containing at least six jets in the final state when one of the $\mathrm{Z}$ bosons decays leptonically and the other decays hadronically. Therefore, most of the sensitivity of the on- $Z$ search is provided by the high jet multiplicity SRs. All of the on-Z strong-production SRs are considered, however, to set limits in this model. The expected and observed limits are presented in figure 7 as a function of the $\widetilde{g}$ and $\widetilde{\chi}_{1}^{0}$ masses. We are able to probe gluino masses up to $1500-1770 \mathrm{GeV}$ depending on the mass of $\tilde{\chi}_{1}^{0}$. This represents an improvement of around $500 \mathrm{GeV}$ compared to the previously published CMS result [14].

The on-Z search for EW production is interpreted using the models described in section 2. For the model of $\widetilde{\chi}_{1}^{ \pm} \widetilde{\chi}_{2}^{0}$ production with decays to WZ, the VZ SR provides almost all of the sensitivity. Figure 8 shows the cross section upper limits and the exclusion lines at $95 \% \mathrm{CL}$, as a function of the $\widetilde{\chi}_{1}^{ \pm}$(or $\widetilde{\chi}_{2}^{0}$ ) and $\widetilde{\chi}_{1}^{0}$ masses. The analysis probes $\widetilde{\chi}_{1}^{ \pm}$masses between approximately 160 and $610 \mathrm{GeV}$, depending on the mass of $\widetilde{\chi}_{1}^{0}$. The observed limit is stronger than expected due to the observed yields being smaller than predicted in the highest two $p_{\mathrm{T}}^{\text {miss }}$ bins of the VZ SR. This result extends the observed exclusion using $8 \mathrm{TeV}$ data by around $300 \mathrm{GeV}$ in the mass of $\widetilde{\chi}_{1}^{ \pm}[17]$. 


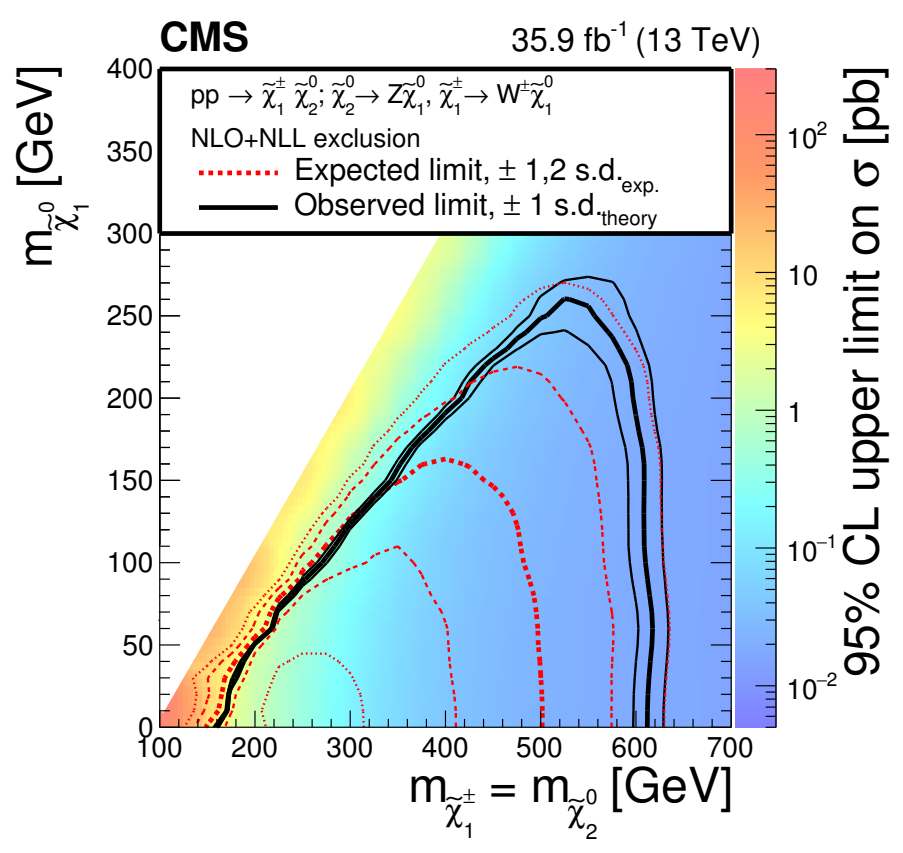

Figure 8. Cross section upper limit and exclusion contours at 95\% CL for the EW WZ model as a function of the $\widetilde{\chi}_{1}^{ \pm}$(equal to $\widetilde{\chi}_{2}^{0}$ ) and $\widetilde{\chi}_{1}^{0}$ masses, obtained using the on- $Z$ search for EW production results. The region under the thick red dotted (black solid) line is excluded by the expected (observed) limit. The thin red dotted curves indicate the regions containing 68 and $95 \%$ of the distribution of limits expected under the background-only hypothesis. The thin solid black curves show the change in the observed limit due to variation of the signal cross sections within their theoretical uncertainties.

For the model of $\widetilde{\chi}_{1}^{0} \widetilde{\chi}_{1}^{0}$ production with decays to $\mathrm{ZZ}$, the $\mathrm{VZ}$ region contains most of the signal, but the HZ SR accepts the events where the $\mathrm{Z}$ boson decays to $\mathrm{b} \overline{\mathrm{b}}$. The limit is shown in figure 9 (upper) as a function of the $\widetilde{\chi}_{1}^{0}$ mass. We probe masses up to around $650 \mathrm{GeV}$. The observed limit is stronger than the expected due to the deficit of observed events in the high- $p_{\mathrm{T}}^{\text {miss }}$ bins of the VZ SR. This result extends the observed limit by around $300 \mathrm{GeV}$ compared to the result using $8 \mathrm{TeV}$ data [18].

For the model of $\widetilde{\chi}_{1}^{0} \widetilde{\chi}_{1}^{0}$ production with decays to HZ, the HZ SR dominates the expected limit. The maximal branching fraction to the $\mathrm{HZ}$ final state is $50 \%$, achieved when $\widetilde{\chi}_{1}^{0}$ decays with $50 \%$ probability to either the $\mathrm{Z}$ or Higgs boson. In this scenario, one also expects to have a $25 \%$ branching fraction to the $\mathrm{ZZ}$ topology. We set limits on the $50 \%$ branching fraction model in figure 9 (lower) using these assumptions and considering the signal contributions from both the $\mathrm{ZZ}$ and $\mathrm{HZ}$ topologies. In this mixed decay model, we probe masses up to around $500 \mathrm{GeV}$. The observed limit at high masses is dominated by the same effect as in the pure $\mathrm{ZZ}$ topology. For masses below $200 \mathrm{GeV}$, the events from the HZ topology alone give an expected exclusion that is 2-5 times more stringent than those from the ZZ topology alone, while for higher masses, the two topologies yield expected limits that are similar to within $30 \%$. The previous exclusion limit using $8 \mathrm{TeV}$ data is extended by around $200 \mathrm{GeV}$. 

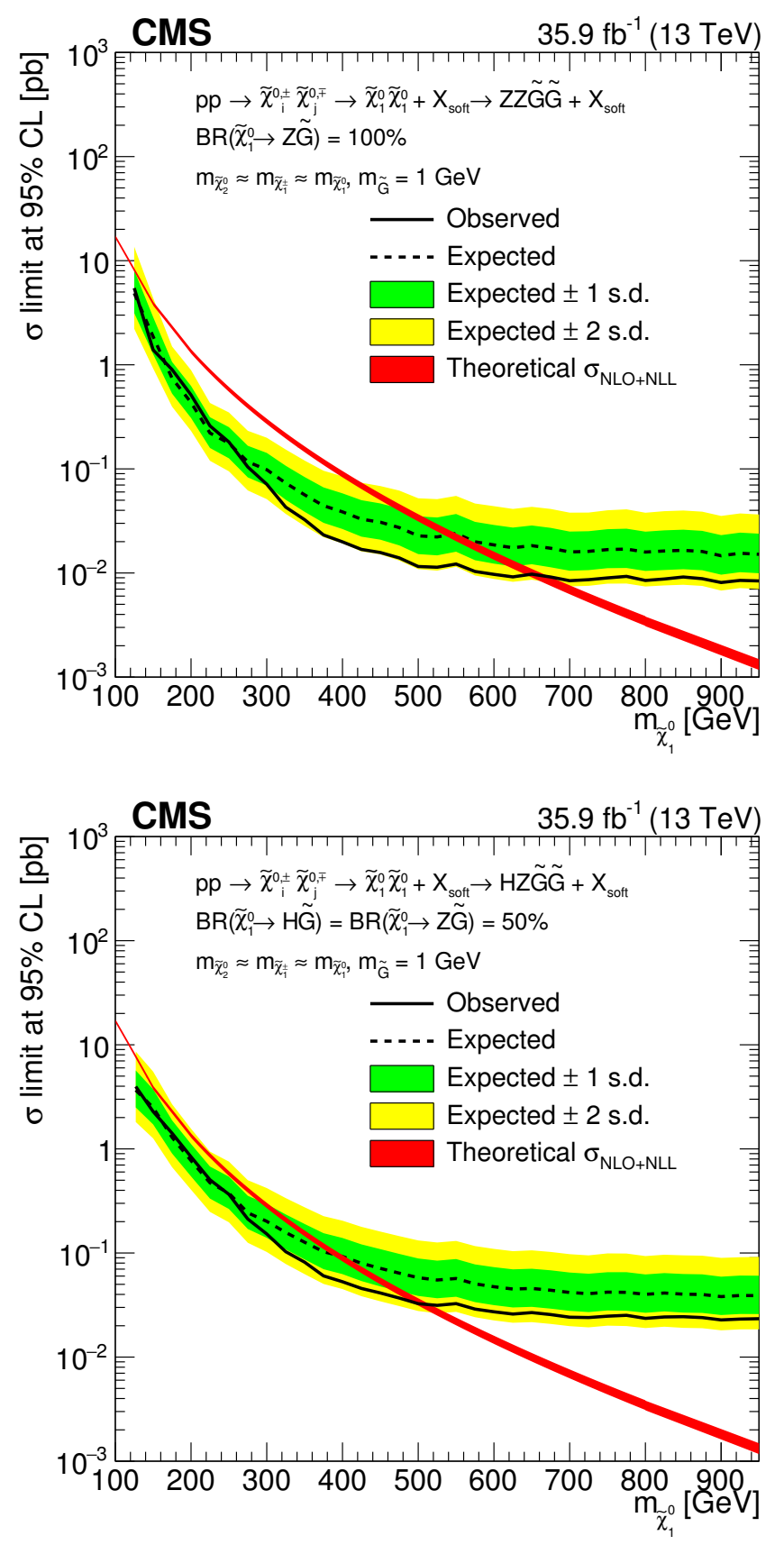

Figure 9. Cross section upper limit and exclusion lines at $95 \% \mathrm{CL}$, as a function of the $\widetilde{\chi}_{1}^{0}$ mass, for the search for EW production in the ZZ topology (upper) and with a $50 \%$ branching fraction to each of the $\mathrm{Z}$ and Higgs bosons (lower). The red band shows the theoretical cross section, with the thickness of band representing the theoretical uncertainty in the signal cross section. Regions where the black dotted line reaches below the theoretical cross section are expected to be excluded. The green (yellow) band indicates the region containing 68 (95)\% of the distribution of limits expected under the background-only hypothesis. The observed upper limit on the cross section is shown with a solid black line. 


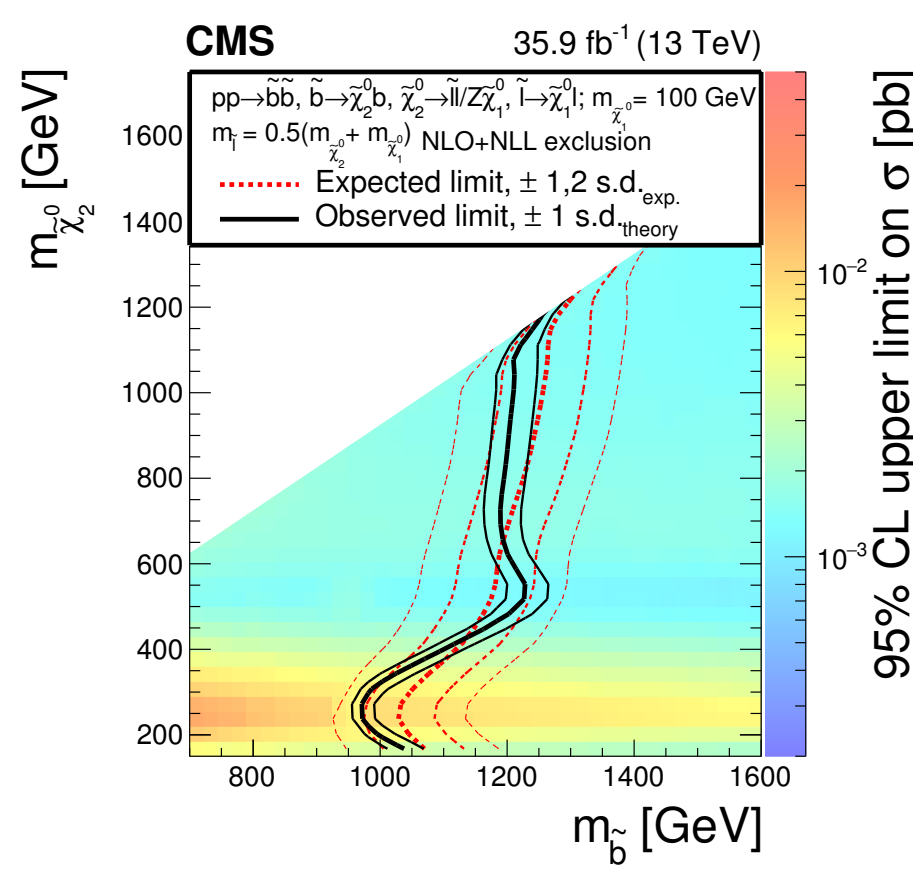

Figure 10. Cross section upper limit and exclusion contours at 95\% CL for the slepton edge model as a function of the $\widetilde{\mathrm{b}}$ and $\widetilde{\chi}_{2}^{0}$ masses, obtained from the results of the edge search. The region to the left of the thick red dotted (black solid) line is excluded by the expected (observed) limit. The thin red dotted curves indicate the regions containing 68 and $95 \%$ of the distribution of limits expected under the background-only hypothesis. The thin solid black curves show the change in the observed limit due to variation of the signal cross sections within their theoretical uncertainties.

The edge search is interpreted using the slepton edge model, combining the seven $m_{\ell \ell}$ bins and the two likelihood regions. Figure 10 shows the exclusion contour as a function of the $\widetilde{\mathrm{b}}$ and $\widetilde{\chi}_{2}^{0}$ masses. We exclude $\widetilde{\mathrm{b}}$ masses up to around $980-1200 \mathrm{GeV}$, depending on the mass of $\widetilde{\chi}_{2}^{0}$, extending previous exclusion limits in the same model by $400-600 \mathrm{GeV}$. A decrease of the sensitivity is observed for those models where the $\widetilde{\chi}_{2}^{0}$ mass is in the range 200-300 GeV. The $m_{\ell \ell}$ distribution for these models has an edge in the range $100-200 \mathrm{GeV}$, and most of the signal events fall either into the SRs with the highest background prediction or in the range $86<m_{\ell \ell}<96 \mathrm{GeV}$, which is not considered for this part of the analysis. The observed limit in this regime is weaker than the expected one due to the deviation in the not-t $\overline{\mathrm{t}}$-like, $96-150 \mathrm{GeV}$ mass bin. For high $\widetilde{\chi}_{2}^{0}$ masses, the majority of signal events fall into the highest mass bins, which are nearly background free. This results in increased

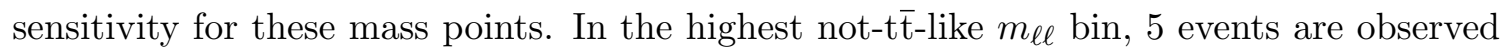

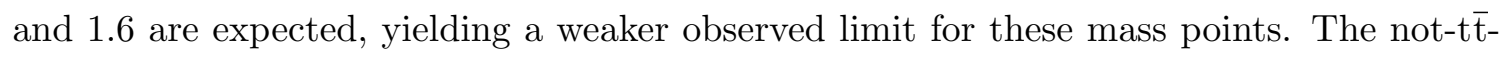
like $m_{\ell \ell}$ bin of $300-400 \mathrm{GeV}$ contains 0 observed events compared to an expectation of 3.5 events, yielding the stronger observed limit for the $\widetilde{\chi}_{2}^{0}$ masses of about $500 \mathrm{GeV}$. 


\section{Summary}

A search for phenomena beyond the standard model (SM) in events with opposite-charge, same-flavor leptons, jets, and missing transverse momentum has been presented. The data used corresponds to a sample of pp collisions collected with the CMS detector in 2016 at a center-of-mass energy of $13 \mathrm{TeV}$, corresponding to an integrated luminosity of $35.9 \mathrm{fb}^{-1}$. Searches are performed for signals with a dilepton invariant mass $\left(m_{\ell \ell}\right)$ compatible with the $\mathrm{Z}$ boson or producing a kinematic edge in the distribution of $m_{\ell \ell}$. By comparing the observation to estimates for SM backgrounds obtained from data control samples, no statistically significant evidence for a signal has been observed.

The search for strongly produced new physics containing an on-shell $\mathrm{Z}$ boson is interpreted in a model of gauge-mediated supersymmetry breaking (GMSB), where the Z bosons are produced in decay chains initiated through gluino pair production. Gluino masses below $1500-1770 \mathrm{GeV}$ have been excluded, depending on the neutralino mass, extending the exclusion limits derived from the previous CMS publication by almost $500 \mathrm{GeV}$.

The search for electroweak production with an on-shell Z boson has been interpreted in multiple simplified models. For chargino-neutralino production, where the neutralino decays to a $\mathrm{Z}$ boson and the lightest supersymmetric particle (LSP) and the chargino decays to a $\mathrm{W}$ boson and the LSP, we probe chargino masses in the range $160-610 \mathrm{GeV}$. In a GMSB model of neutralino-neutralino production decaying to ZZ and LSPs, we probe neutralino masses up to around $650 \mathrm{GeV}$. Assuming GMSB production where the neutralino has a branching fraction of $50 \%$ to the $\mathrm{Z}$ boson and $50 \%$ to the Higgs boson, we probe neutralino masses up to around $500 \mathrm{GeV}$. Compared to published CMS results using $8 \mathrm{TeV}$ data, these extend the exclusion limits by around $200-300 \mathrm{GeV}$ depending on the model.

The search for a kinematic edge in the $m_{\ell \ell}$ distribution is interpreted in a simplified model based on bottom squark pair production. Decay chains containing the two lightest neutralinos and a slepton are assumed, leading to edge-like signatures in the distribution of $m_{\ell \ell}$. Bottom squark masses below $980-1200 \mathrm{GeV}$ have been excluded, depending on the mass of the second neutralino. These extend the previous CMS exclusion limits in the same model by $400-600 \mathrm{GeV}$.

\section{Acknowledgments}

We congratulate our colleagues in the CERN accelerator departments for the excellent performance of the LHC and thank the technical and administrative staffs at CERN and at other CMS institutes for their contributions to the success of the CMS effort. In addition, we gratefully acknowledge the computing centers and personnel of the Worldwide LHC Computing Grid for delivering so effectively the computing infrastructure essential to our analyses. Finally, we acknowledge the enduring support for the construction and operation of the LHC and the CMS detector provided by the following funding agencies: BMWFW and FWF (Austria); FNRS and FWO (Belgium); CNPq, CAPES, FAPERJ, and FAPESP (Brazil); MES (Bulgaria); CERN; CAS, MoST, and NSFC (China); COL- 
CIENCIAS (Colombia); MSES and CSF (Croatia); RPF (Cyprus); SENESCYT (Ecuador); MoER, ERC IUT, and ERDF (Estonia); Academy of Finland, MEC, and HIP (Finland); CEA and CNRS/IN2P3 (France); BMBF, DFG, and HGF (Germany); GSRT (Greece); OTKA and NIH (Hungary); DAE and DST (India); IPM (Iran); SFI (Ireland); INFN (Italy); MSIP and NRF (Republic of Korea); LAS (Lithuania); MOE and UM (Malaysia); BUAP, CINVESTAV, CONACYT, LNS, SEP, and UASLP-FAI (Mexico); MBIE (New Zealand); PAEC (Pakistan); MSHE and NSC (Poland); FCT (Portugal); JINR (Dubna); MON, RosAtom, RAS, RFBR and RAEP (Russia); MESTD (Serbia); SEIDI, CPAN, PCTI and FEDER (Spain); Swiss Funding Agencies (Switzerland); MST (Taipei); ThEPCenter, IPST, STAR, and NSTDA (Thailand); TUBITAK and TAEK (Turkey); NASU and SFFR (Ukraine); STFC (United Kingdom); DOE and NSF (U.S.A.).

Individuals have received support from the Marie-Curie program and the European Research Council and Horizon 2020 Grant, contract No. 675440 (European Union); the Leventis Foundation; the A. P. Sloan Foundation; the Alexander von Humboldt Foundation; the Belgian Federal Science Policy Office; the Fonds pour la Formation à la Recherche dans l'Industrie et dans l'Agriculture (FRIA-Belgium); the Agentschap voor Innovatie door Wetenschap en Technologie (IWT-Belgium); the Ministry of Education, Youth and Sports (MEYS) of the Czech Republic; the Council of Science and Industrial Research, India; the HOMING PLUS program of the Foundation for Polish Science, cofinanced from European Union, Regional Development Fund, the Mobility Plus program of the Ministry of Science and Higher Education, the National Science Center (Poland), contracts Harmonia 2014/14/M/ST2/00428, Opus 2014/13/B/ST2/02543, 2014/15/B/ST2/03998, and 2015/19/B/ST2/02861, Sonata-bis 2012/07/E/ST2/01406; the National Priorities Research Program by Qatar National Research Fund; the Programa Severo Ochoa del Principado de Asturias; the Thalis and Aristeia programs cofinanced by EU-ESF and the Greek NSRF; the Rachadapisek Sompot Fund for Postdoctoral Fellowship, Chulalongkorn University and the Chulalongkorn Academic into Its 2nd Century Project Advancement Project (Thailand); the Welch Foundation, contract C-1845; and the Weston Havens Foundation (U.S.A.).

\section{A Correlation and covariance matrices for the background predictions}

In order to facilitate the interpretation of the analysis results in other models, we provide the covariance and correlation matrices for the background predictions in the different SRs. Figure 11 shows a graphical representation of the covariance (upper) and correlation (lower) matrices for the on-Z strong-production SRs. Figure 12 shows the same matrices for the on-Z electroweak-production SRs, and figure 13 shows the corresponding matrices for the edge strong-production SRs. Because of potential overlaps in selected events, only the strong- or electroweak-production on-Z SRs should be used for interpretation, not both sets simultaneously. This information can be used to construct a simplified likelihood for models of new physics, as described in ref. [67]. 


\section{CMS}

$35.9 \mathrm{fb}^{-1}(13 \mathrm{TeV})$
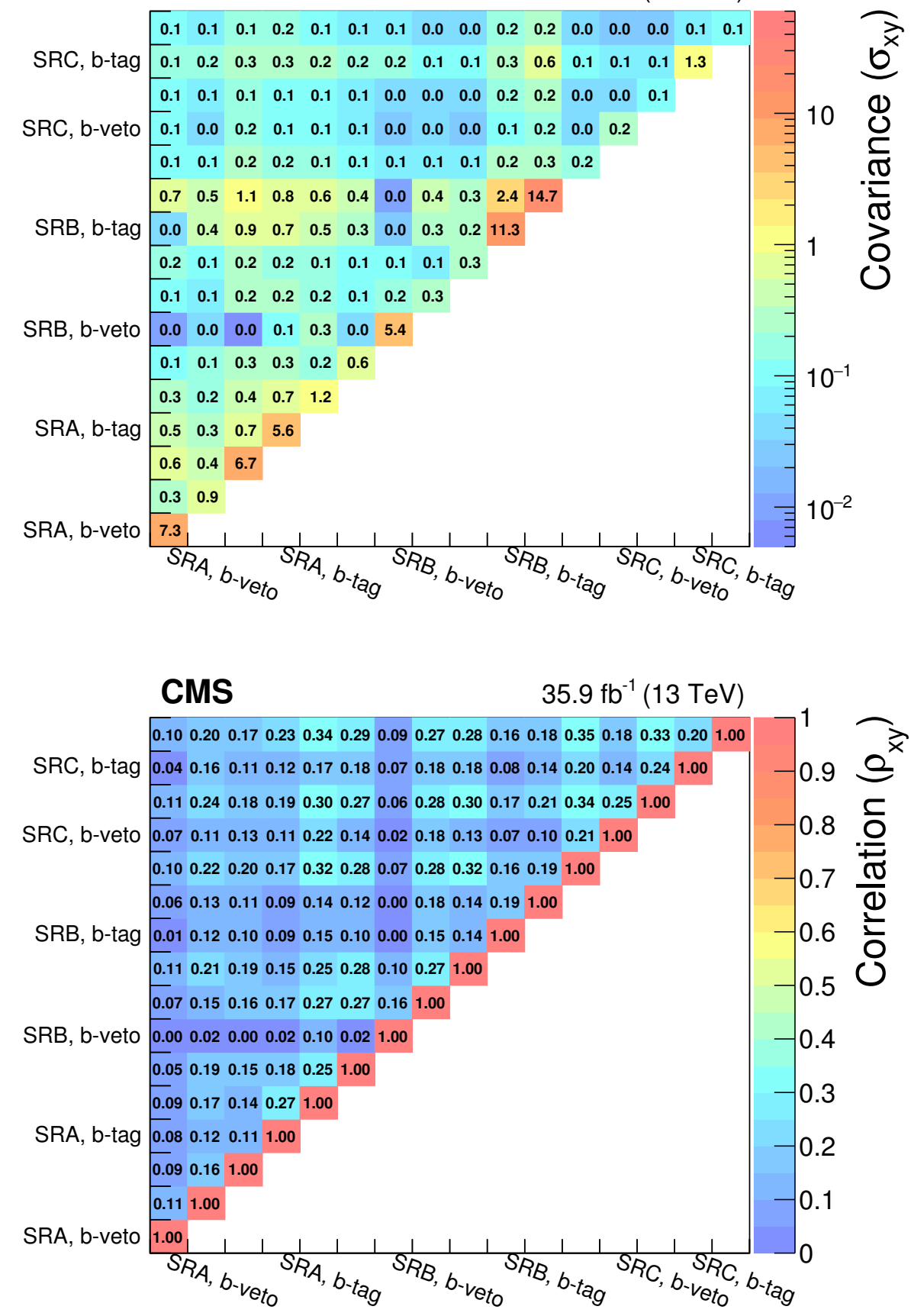

Figure 11. The covariance (upper) and correlation (lower) matrices for the background predictions in the on-Z strong-production SRs. Within each SR, the individual $p_{\mathrm{T}}^{\mathrm{miss}}$ bins are shown in increasing order starting from $100 \mathrm{GeV}$. The matrices are symmetric, but only the entries along and above the diagonal are shown for simplicity. 

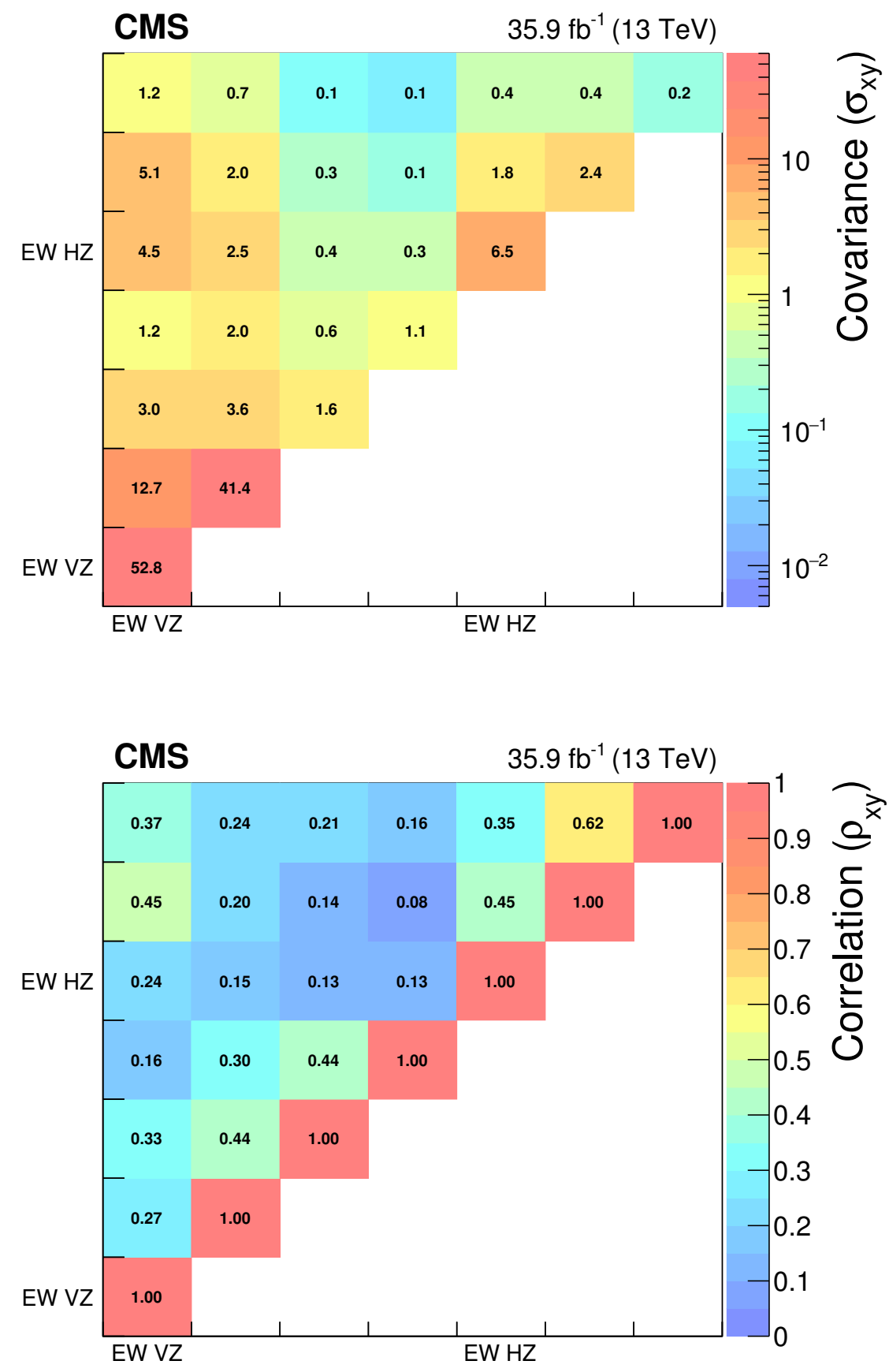

Figure 12. The covariance (upper) and correlation (lower) matrices for the background predictions in the on-Z EW-production SRs. Within each SR, the individual $p_{\mathrm{T}}^{\text {miss }}$ bins are shown in increasing order starting from $100 \mathrm{GeV}$. The matrices are symmetric, but only the entries along and above the diagonal are shown for simplicity. 


\section{CMS}
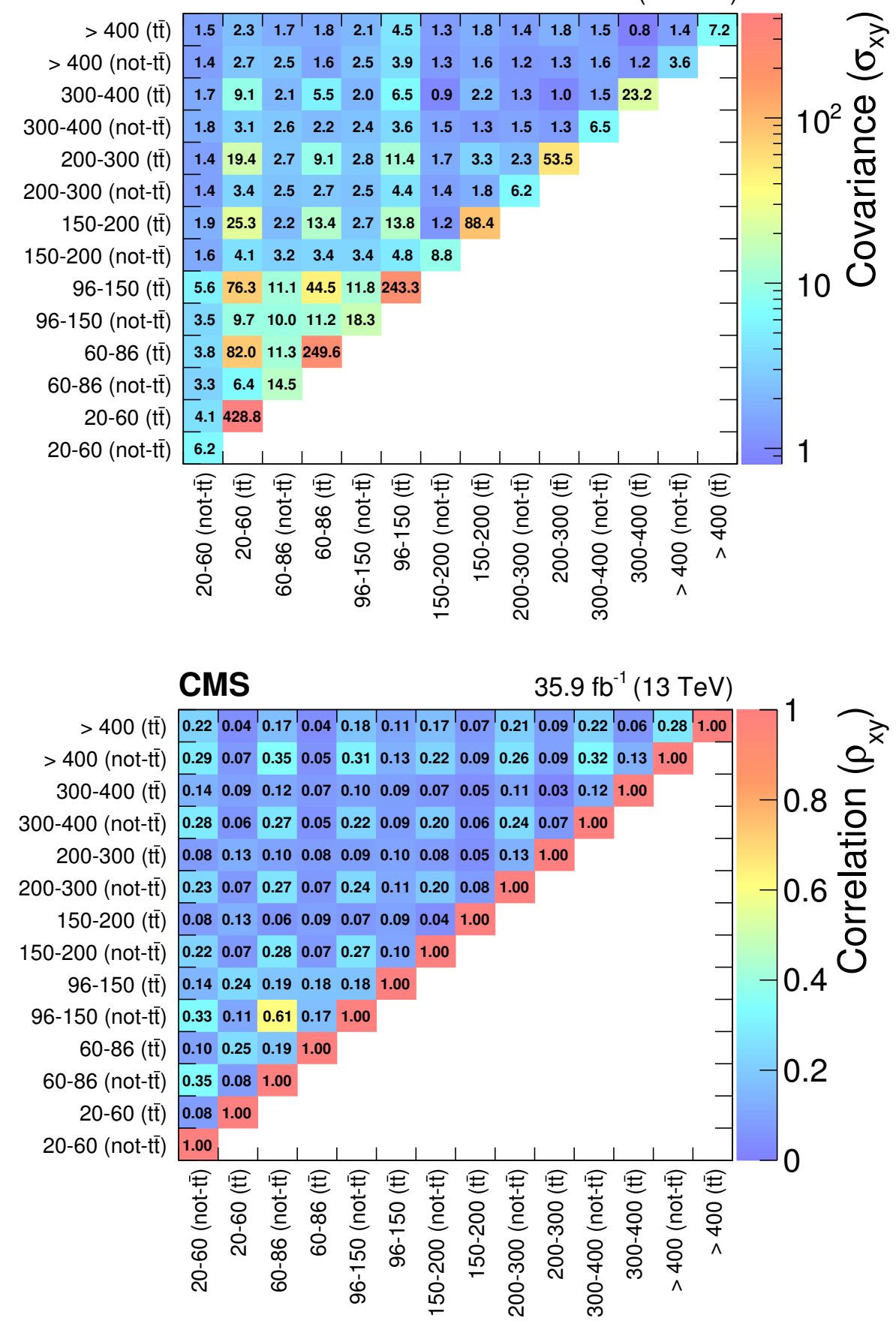

Figure 13. The covariance (upper) and correlation (lower) matrices for the background predictions in the edge strong-production SRs. The matrices are symmetric, but only the entries along and above the diagonal are shown for simplicity.

Open Access. This article is distributed under the terms of the Creative Commons Attribution License (CC-BY 4.0), which permits any use, distribution and reproduction in any medium, provided the original author(s) and source are credited. 


\section{References}

[1] P. Ramond, Dual theory for free fermions, Phys. Rev. D 3 (1971) 2415 [InSPIRE].

[2] Yu. A. Golfand and E.P. Likhtman, Extension of the algebra of Poincaré group generators and violation of $p$ invariance, JETP Lett. 13 (1971) 323 [Pisma Zh. Eksp. Teor. Fiz. 13 (1971) 452] [INSPIRE].

[3] A. Neveu and J.H. Schwarz, Factorizable dual model of pions, Nucl. Phys. B 31 (1971) 86 [INSPIRE].

[4] D.V. Volkov and V.P. Akulov, Possible universal neutrino interaction, JETP Lett. 16 (1972) 438 [Pisma Zh. Eksp. Teor. Fiz. 16 (1972) 621] [InSPIRE].

[5] J. Wess and B. Zumino, A Lagrangian model invariant under supergauge transformations, Phys. Lett. B 49 (1974) 52 [inSPIRE].

[6] J. Wess and B. Zumino, Supergauge transformations in four-dimensions, Nucl. Phys. B 70 (1974) 39 [INSPIRE].

[7] P. Fayet, Supergauge invariant extension of the Higgs mechanism and a model for the electron and its neutrino, Nucl. Phys. B 90 (1975) 104 [INSPIRE].

[8] H.P. Nilles, Supersymmetry, supergravity and particle physics, Phys. Rept. 110 (1984) 1 [INSPIRE].

[9] G.R. Farrar and P. Fayet, Phenomenology of the production, decay and detection of new hadronic states associated with supersymmetry, Phys. Lett. B 76 (1978) 575 [INSPIRE].

[10] A.J. Buras, J.R. Ellis, M.K. Gaillard and D.V. Nanopoulos, Aspects of the grand unification of strong, weak and electromagnetic interactions, Nucl. Phys. B 135 (1978) 66 [InSPIRE].

[11] H.E. Haber and G.L. Kane, The search for supersymmetry: probing physics beyond the Standard Model, Phys. Rept. 117 (1985) 75 [INSPIRE].

[12] I. Hinchliffe, F.E. Paige, M.D. Shapiro, J. Soderqvist and W. Yao, Precision SUSY measurements at CERN LHC, Phys. Rev. D 55 (1997) 5520 [hep-ph/9610544] [INSPIRE].

[13] CMS collaboration, Search for physics beyond the Standard Model in events with two leptons, jets and missing transverse momentum in pp collisions at $\sqrt{s}=8 \mathrm{TeV}, \mathrm{JHEP} \mathbf{0 4}$ (2015) 124 [arXiv:1502.06031] [INSPIRE].

[14] CMS collaboration, Search for new physics in final states with two opposite-sign, same-flavor leptons, jets and missing transverse momentum in pp collisions at $\sqrt{s}=13$ TeV, JHEP 12 (2016) 013 [arXiv:1607.00915] [INSPIRE].

[15] CMS collaboration, Search for new physics in events with opposite-sign leptons, jets and missing transverse energy in pp collisions at $\sqrt{s}=7$ TeV, Phys. Lett. B 718 (2013) 815 [arXiv: 1206.3949] [INSPIRE].

[16] CMS collaboration, Search for physics beyond the Standard Model in opposite-sign dilepton events at $\sqrt{s}=7 \mathrm{TeV}$, JHEP 06 (2011) 026 [arXiv:1103.1348] [INSPIRE].

[17] CMS collaboration, Searches for electroweak production of charginos, neutralinos and sleptons decaying to leptons and $W, Z$ and Higgs bosons in pp collisions at 8 TeV, Eur. Phys. J. C 74 (2014) 3036 [arXiv:1405.7570] [INSPIRE]. 
[18] CMS collaboration, Searches for electroweak neutralino and chargino production in channels with Higgs, $Z$ and $W$ bosons in pp collisions at 8 TeV, Phys. Rev. D 90 (2014) 092007 [arXiv: 1409.3168] [INSPIRE].

[19] ATLAS collaboration, Search for supersymmetry in events containing a same-flavour opposite-sign dilepton pair, jets and large missing transverse momentum in $\sqrt{s}=8$ TeV pp collisions with the ATLAS detector, Eur. Phys. J. C 75 (2015) 318 [Erratum ibid. C 75 (2015) 463] [arXiv:1503.03290] [INSPIRE].

[20] ATLAS collaboration, Search for the electroweak production of supersymmetric particles in $\sqrt{s}=8$ TeV pp collisions with the ATLAS detector, Phys. Rev. D 93 (2016) 052002 [arXiv:1509.07152] [INSPIRE].

[21] ATLAS collaboration, Search for new phenomena in events containing a same-flavour opposite-sign dilepton pair, jets and large missing transverse momentum in $\sqrt{s}=13$ TeV $\mathrm{pp}$ collisions with the ATLAS detector, Eur. Phys. J. C 77 (2017) 144 [arXiv:1611.05791] [INSPIRE].

[22] N. Arkani-Hamed et al., MARMOSET: the path from LHC data to the new Standard Model via on-shell effective theories, hep-ph/0703088 [INSPIRE].

[23] J. Alwall, P. Schuster and N. Toro, Simplified models for a first characterization of new physics at the LHC, Phys. Rev. D 79 (2009) 075020 [arXiv:0810.3921] [INSPIRE].

[24] J. Alwall, M.-P. Le, M. Lisanti and J.G. Wacker, Model-independent jets plus missing energy searches, Phys. Rev. D 79 (2009) 015005 [arXiv:0809.3264] [InSPIRE].

[25] LHC New Physics Working Group collaboration, D. Alves, Simplified models for LHC new physics searches, J. Phys. G 39 (2012) 105005 [arXiv:1105.2838] [INSPIRE].

[26] CMS collaboration, Interpretation of searches for supersymmetry with simplified models, Phys. Rev. D 88 (2013) 052017 [arXiv: 1301.2175] [InSPIRE].

[27] K.T. Matchev and S.D. Thomas, Higgs and $Z$ boson signatures of supersymmetry, Phys. Rev. D 62 (2000) 077702 [hep-ph/9908482] [INSPIRE].

[28] P. Meade, M. Reece and D. Shih, Prompt decays of general neutralino NLSPs at the Tevatron, JHEP 05 (2010) 105 [arXiv:0911.4130] [INSPIRE].

[29] J.T. Ruderman and D. Shih, General neutralino NLSPs at the early LHC, JHEP 08 (2012) 159 [arXiv: 1103.6083] [INSPIRE].

[30] P.Z. Skands et al., SUSY Les Houches accord: interfacing SUSY spectrum calculators, decay packages and event generators, JHEP 07 (2004) 036 [hep-ph/0311123] [INSPIRE].

[31] CMS collaboration, The CMS experiment at the CERN LHC, 2008 JINST 3 S08004 [INSPIRE].

[32] CMS collaboration, Particle-flow reconstruction and global event description with the CMS detector, 2017 JINST 12 P10003 [arXiv:1706.04965] [INSPIRE].

[33] M. Cacciari, G.P. Salam and G. Soyez, The anti- $k_{t}$ jet clustering algorithm, JHEP 04 (2008) 063 [arXiv:0802.1189] [INSPIRE].

[34] M. Cacciari, G.P. Salam and G. Soyez, FastJet user manual, Eur. Phys. J. C 72 (2012) 1896 [arXiv:1111.6097] [INSPIRE]. 
[35] CMS collaboration, Performance of electron reconstruction and selection with the CMS detector in proton-proton collisions at $\sqrt{s}=8 \mathrm{TeV}, 2015$ JINST $10 \mathrm{P} 06005$ [arXiv: 1502.02701] [INSPIRE].

[36] K. Rehermann and B. Tweedie, Efficient identification of boosted semileptonic top quarks at the LHC, JHEP 03 (2011) 059 [arXiv: 1007.2221] [INSPIRE].

[37] CMS collaboration, Performance of photon reconstruction and identification with the CMS detector in proton-proton collisions at $\sqrt{s}=8 \mathrm{TeV}, 2015$ JINST $10 \mathrm{P} 08010$ [arXiv: 1502.02702] [INSPIRE].

[38] M. Cacciari and G.P. Salam, Dispelling the $N^{3}$ myth for the $k_{t}$ jet-finder, Phys. Lett. B 641 (2006) 57 [hep-ph/0512210] [INSPIRE].

[39] CMS collaboration, Determination of jet energy calibration and transverse momentum resolution in CMS, 2011 JINST 6 P11002 [arXiv: 1107.4277] [INSPIRE].

[40] M. Cacciari and G.P. Salam, Pileup subtraction using jet areas, Phys. Lett. B 659 (2008) 119 [arXiv:0707.1378] [INSPIRE].

[41] CMS collaboration, Identification of heavy-flavour jets with the CMS detector in pp collisions at $13 \mathrm{TeV}$, submitted to JINST (2017) [arXiv:1712.07158] [INSPIRE].

[42] J. Alwall et al., The automated computation of tree-level and next-to-leading order differential cross sections and their matching to parton shower simulations, JHEP 07 (2014) 079 [arXiv: 1405.0301] [INSPIRE].

[43] S. Alioli, P. Nason, C. Oleari and E. Re, NLO single-top production matched with shower in POWHEG: s-and t-channel contributions, JHEP 09 (2009) 111 [Erratum ibid. 02 (2010) 011] [arXiv:0907.4076] [INSPIRE].

[44] E. Re, Single-top Wt-channel production matched with parton showers using the POWHEG method, Eur. Phys. J. C 71 (2011) 1547 [arXiv: 1009.2450] [INSPIRE].

[45] R. Gavin, Y. Li, F. Petriello and S. Quackenbush, FEWZ 2.0: a code for hadronic Z production at next-to-next-to-leading order, Comput. Phys. Commun. 182 (2011) 2388 [arXiv:1011.3540] [INSPIRE].

[46] R. Gavin, Y. Li, F. Petriello and S. Quackenbush, W physics at the LHC with FEWZ 2.1, Comput. Phys. Commun. 184 (2013) 208 [arXiv:1201.5896] [InSPIRE].

[47] M. Czakon and A. Mitov, Top++: a program for the calculation of the top-pair cross-section at hadron colliders, Comput. Phys. Commun. 185 (2014) 2930 [arXiv:1112.5675] [INSPIRE].

[48] C. Borschensky et al., Squark and gluino production cross sections in pp collisions at $\sqrt{s}=13,14,33$ and 100 TeV, Eur. Phys. J. C 74 (2014) 3174 [arXiv:1407.5066] [InSPIRE].

[49] B. Fuks, M. Klasen, D.R. Lamprea and M. Rothering, Gaugino production in proton-proton collisions at a center-of-mass energy of 8 TeV, JHEP 10 (2012) 081 [arXiv:1207.2159] [INSPIRE].

[50] B. Fuks, M. Klasen, D.R. Lamprea and M. Rothering, Precision predictions for electroweak superpartner production at hadron colliders with resummino, Eur. Phys. J. C 73 (2013) 2480 [arXiv: 1304.0790] [INSPIRE].

[51] J. Alwall et al., Comparative study of various algorithms for the merging of parton showers and matrix elements in hadronic collisions, Eur. Phys. J. C 53 (2008) 473 [arXiv:0706.2569] [INSPIRE]. 
[52] S. Frixione, P. Nason and C. Oleari, Matching NLO QCD computations with parton shower simulations: the POWHEG method, JHEP 11 (2007) 070 [arXiv:0709.2092] [INSPIRE].

[53] T. Sjöstrand, S. Mrenna and P.Z. Skands, A brief introduction to PYTHIA 8.1, Comput. Phys. Commun. 178 (2008) 852 [arXiv:0710.3820] [INSPIRE].

[54] NNPDF collaboration, R.D. Ball et al., Parton distributions for the LHC run II, JHEP 04 (2015) 040 [arXiv: 1410.8849] [INSPIRE].

[55] GEANT4 collaboration, S. Agostinelli et al., GEANT4: a simulation toolkit, Nucl. Instrum. Meth. A 506 (2003) 250 [INSPIRE].

[56] CMS collaboration, S. Abdullin, P. Azzi, F. Beaudette, P. Janot and A. Perrotta, The fast simulation of the CMS detector at LHC, J. Phys. Conf. Ser. 331 (2011) 032049 [InSPIRE].

[57] C.G. Lester and D.J. Summers, Measuring masses of semiinvisibly decaying particles pair produced at hadron colliders, Phys. Lett. B 463 (1999) 99 [hep-ph/9906349] [INSPIRE].

[58] A. Barr, C. Lester and P. Stephens, A variable for measuring masses at hadron colliders when missing energy is expected; $m_{T 2}$ : the truth behind the glamour, J. Phys. G 29 (2003) 2343 [hep-ph/0304226] [INSPIRE].

[59] M.J. Oreglia, A study of the reactions $\psi^{\prime} \rightarrow \gamma \gamma \psi$, appendix D, Ph.D. thesis, SLAC report SLAC-R-236, Stanford University, U.S.A., (1980) [INSPIRE].

[60] Particle Data Group collaboration, C. Patrignani et al., Review of particle physics, Chin. Phys. C 40 (2016) 100001 [inSPIRE].

[61] E. Gross and O. Vitells, Trial factors for the look elsewhere effect in high energy physics, Eur. Phys. J. C 70 (2010) 525 [arXiv: 1005.1891] [InSPIRE].

[62] T. Junk, Confidence level computation for combining searches with small statistics, Nucl. Instrum. Meth. A 434 (1999) 435 [hep-ex/9902006] [INSPIRE].

[63] A.L. Read, Presentation of search results: the $C L_{s}$ technique, J. Phys. G 28 (2002) 2693 [INSPIRE].

[64] ATLAS, CMS collaborations and the LHC Higgs Combination Group, Procedure for the LHC Higgs boson search combination in Summer 2011, ATL-PHYS-PUB-2011-011, CERN, Geneva Switzerland, (2011) [CMS-NOTE-2011-005].

[65] G. Cowan, K. Cranmer, E. Gross and O. Vitells, Asymptotic formulae for likelihood-based tests of new physics, Eur. Phys. J. C 71 (2011) 1554 [Erratum ibid. C 73 (2013) 2501] [arXiv: 1007.1727] [INSPIRE].

[66] CMS collaboration, CMS luminosity measurements for the 2016 data taking period, CMS-PAS-LUM-17-001, CERN, Geneva Switzerland, (2017).

[67] CMS collaboration, Simplified likelihood for the re-interpretation of public CMS results, CMS-NOTE-2017-001, CERN, Geneva Switzerland, (2017). 


\section{The CMS collaboration}

\section{Yerevan Physics Institute, Yerevan, Armenia}

A.M. Sirunyan, A. Tumasyan

\section{Institut für Hochenergiephysik, Wien, Austria}

W. Adam, F. Ambrogi, E. Asilar, T. Bergauer, J. Brandstetter, E. Brondolin, M. Dragicevic, J. Erö, M. Flechl, M. Friedl, R. Frühwirth ${ }^{1}$, V.M. Ghete, J. Grossmann, J. Hrubec, M. Jeitler ${ }^{1}$, A. König, N. Krammer, I. Krätschmer, D. Liko, T. Madlener, I. Mikulec, E. Pree, D. Rabady, N. Rad, H. Rohringer, J. Schieck ${ }^{1}$, R. Schöfbeck, M. Spanring, D. Spitzbart, W. Waltenberger, J. Wittmann, C.-E. Wulz ${ }^{1}$, M. Zarucki

Institute for Nuclear Problems, Minsk, Belarus

V. Chekhovsky, V. Mossolov, J. Suarez Gonzalez

\section{Universiteit Antwerpen, Antwerpen, Belgium}

E.A. De Wolf, D. Di Croce, X. Janssen, J. Lauwers, M. Van De Klundert, H. Van Haevermaet, P. Van Mechelen, N. Van Remortel

\section{Vrije Universiteit Brussel, Brussel, Belgium}

S. Abu Zeid, F. Blekman, J. D’Hondt, I. De Bruyn, J. De Clercq, K. Deroover, G. Flouris, D. Lontkovskyi, S. Lowette, S. Moortgat, L. Moreels, Q. Python, K. Skovpen, S. Tavernier, W. Van Doninck, P. Van Mulders, I. Van Parijs

\section{Université Libre de Bruxelles, Bruxelles, Belgium}

H. Brun, B. Clerbaux, G. De Lentdecker, H. Delannoy, G. Fasanella, L. Favart, R. Goldouzian, A. Grebenyuk, G. Karapostoli, T. Lenzi, J. Luetic, T. Maerschalk, A. Marinov, A. Randle-conde, T. Seva, C. Vander Velde, P. Vanlaer, D. Vannerom, R. Yonamine, F. Zenoni, F. Zhang ${ }^{2}$

\section{Ghent University, Ghent, Belgium}

A. Cimmino, T. Cornelis, D. Dobur, A. Fagot, M. Gul, I. Khvastunov, D. Poyraz, C. Roskas, S. Salva, M. Tytgat, W. Verbeke, N. Zaganidis

\section{Université Catholique de Louvain, Louvain-la-Neuve, Belgium}

H. Bakhshiansohi, O. Bondu, S. Brochet, G. Bruno, C. Caputo, A. Caudron, S. De Visscher, C. Delaere, M. Delcourt, B. Francois, A. Giammanco, A. Jafari, M. Komm, G. Krintiras, V. Lemaitre, A. Magitteri, A. Mertens, M. Musich, K. Piotrzkowski, L. Quertenmont, M. Vidal Marono, S. Wertz

\section{Université de Mons, Mons, Belgium}

N. Beliy

\section{Centro Brasileiro de Pesquisas Fisicas, Rio de Janeiro, Brazil}

W.L. Aldá Júnior, F.L. Alves, G.A. Alves, L. Brito, M. Correa Martins Junior, C. Hensel, A. Moraes, M.E. Pol, P. Rebello Teles 
Universidade do Estado do Rio de Janeiro, Rio de Janeiro, Brazil

E. Belchior Batista Das Chagas, W. Carvalho, J. Chinellato ${ }^{3}$, A. Custódio, E.M. Da Costa, G.G. Da Silveira ${ }^{4}$, D. De Jesus Damiao, S. Fonseca De Souza, L.M. Huertas Guativa, H. Malbouisson, M. Melo De Almeida, C. Mora Herrera, L. Mundim, H. Nogima, A. Santoro, A. Sznajder, E.J. Tonelli Manganote ${ }^{3}$, F. Torres Da Silva De Araujo, A. Vilela Pereira

Universidade Estadual Paulista $^{a}$, Universidade Federal do ABC ${ }^{b}$, São Paulo, Brazil

S. Ahuja ${ }^{a}$, C.A. Bernardes ${ }^{a}$, T.R. Fernandez Perez Tomei ${ }^{a}$, E.M. Gregores ${ }^{b}$, P.G. Mercadante ${ }^{b}$, S.F. Novaes ${ }^{a}$, Sandra S. Padula ${ }^{a}$, D. Romero $\operatorname{Abad}^{b}$, J.C. Ruiz $\operatorname{Vargas}^{a}$

Institute for Nuclear Research and Nuclear Energy of Bulgaria Academy of Sciences

A. Aleksandrov, R. Hadjiiska, P. Iaydjiev, M. Misheva, M. Rodozov, M. Shopova, S. Stoykova, G. Sultanov

University of Sofia, Sofia, Bulgaria

A. Dimitrov, I. Glushkov, L. Litov, B. Pavlov, P. Petkov

Beihang University, Beijing, China

W. Fang ${ }^{5}$, X. Gao $^{5}$

Institute of High Energy Physics, Beijing, China

M. Ahmad, J.G. Bian, G.M. Chen, H.S. Chen, M. Chen, Y. Chen, C.H. Jiang, D. Leggat, H. Liao, Z. Liu, F. Romeo, S.M. Shaheen, A. Spiezia, J. Tao, C. Wang, Z. Wang, E. Yazgan, H. Zhang, J. Zhao

State Key Laboratory of Nuclear Physics and Technology, Peking University, Beijing, China

Y. Ban, G. Chen, Q. Li, S. Liu, Y. Mao, S.J. Qian, D. Wang, Z. Xu

Universidad de Los Andes, Bogota, Colombia

C. Avila, A. Cabrera, L.F. Chaparro Sierra, C. Florez, C.F. González Hernández, J.D. Ruiz Alvarez

University of Split, Faculty of Electrical Engineering, Mechanical Engineering and Naval Architecture, Split, Croatia

B. Courbon, N. Godinovic, D. Lelas, I. Puljak, P.M. Ribeiro Cipriano, T. Sculac

University of Split, Faculty of Science, Split, Croatia

Z. Antunovic, M. Kovac

Institute Rudjer Boskovic, Zagreb, Croatia

V. Brigljevic, D. Ferencek, K. Kadija, B. Mesic, A. Starodumov ${ }^{6}$, T. Susa

University of Cyprus, Nicosia, Cyprus

M.W. Ather, A. Attikis, G. Mavromanolakis, J. Mousa, C. Nicolaou, F. Ptochos, P.A. Razis, H. Rykaczewski 
Charles University, Prague, Czech Republic

M. Finger ${ }^{7}$, M. Finger Jr. ${ }^{7}$

Universidad San Francisco de Quito, Quito, Ecuador

E. Carrera Jarrin

Academy of Scientific Research and Technology of the Arab Republic of Egypt, Egyptian Network of High Energy Physics, Cairo, Egypt

A. Ellithi Kamel ${ }^{8}$, S. Khalil ${ }^{9}$, A. Mohamed ${ }^{9}$

National Institute of Chemical Physics and Biophysics, Tallinn, Estonia

R.K. Dewanjee, M. Kadastik, L. Perrini, M. Raidal, A. Tiko, C. Veelken

Department of Physics, University of Helsinki, Helsinki, Finland

P. Eerola, J. Pekkanen, M. Voutilainen

Helsinki Institute of Physics, Helsinki, Finland

J. Härkönen, T. Järvinen, V. Karimäki, R. Kinnunen, T. Lampén, K. Lassila-Perini,

S. Lehti, T. Lindén, P. Luukka, E. Tuominen, J. Tuominiemi, E. Tuovinen

Lappeenranta University of Technology, Lappeenranta, Finland

J. Talvitie, T. Tuuva

IRFU, CEA, Université Paris-Saclay, Gif-sur-Yvette, France

M. Besancon, F. Couderc, M. Dejardin, D. Denegri, J.L. Faure, F. Ferri, S. Ganjour, S. Ghosh, A. Givernaud, P. Gras, G. Hamel de Monchenault, P. Jarry, I. Kucher, E. Locci, M. Machet, J. Malcles, G. Negro, J. Rander, A. Rosowsky, M.Ö. Sahin, M. Titov

Laboratoire Leprince-Ringuet, Ecole polytechnique, CNRS/IN2P3, Université Paris-Saclay, Palaiseau, France

A. Abdulsalam, I. Antropov, S. Baffioni, F. Beaudette, P. Busson, L. Cadamuro, C. Charlot, R. Granier de Cassagnac, M. Jo, S. Lisniak, A. Lobanov, J. Martin Blanco, M. Nguyen, C. Ochando, G. Ortona, P. Paganini, P. Pigard, S. Regnard, R. Salerno, J.B. Sauvan, Y. Sirois, A.G. Stahl Leiton, T. Strebler, Y. Yilmaz, A. Zabi, A. Zghiche

Université de Strasbourg, CNRS, IPHC UMR 7178, F-67000 Strasbourg, France

J.-L. Agram ${ }^{10}$, J. Andrea, D. Bloch, J.-M. Brom, M. Buttignol, E.C. Chabert, N. Chanon, C. Collard, E. Conte ${ }^{10}$, X. Coubez, J.-C. Fontaine ${ }^{10}$, D. Gelé, U. Goerlach, M. Jansová, A.-C. Le Bihan, N. Tonon, P. Van Hove

Centre de Calcul de l'Institut National de Physique Nucleaire et de Physique des Particules, CNRS/IN2P3, Villeurbanne, France

S. Gadrat

Université de Lyon, Université Claude Bernard Lyon 1, CNRS-IN2P3, Institut de Physique Nucléaire de Lyon, Villeurbanne, France

S. Beauceron, C. Bernet, G. Boudoul, R. Chierici, D. Contardo, P. Depasse, H. El Mamouni, J. Fay, L. Finco, S. Gascon, M. Gouzevitch, G. Grenier, B. Ille, F. Lagarde, I.B. Laktineh, 
M. Lethuillier, L. Mirabito, A.L. Pequegnot, S. Perries, A. Popov ${ }^{11}$, V. Sordini, M. Vander Donckt, S. Viret

Georgian Technical University, Tbilisi, Georgia

T. Toriashvili ${ }^{12}$

Tbilisi State University, Tbilisi, Georgia

L. Rurua

RWTH Aachen University, I. Physikalisches Institut, Aachen, Germany

C. Autermann, S. Beranek, L. Feld, M.K. Kiesel, K. Klein, M. Lipinski, M. Preuten,

C. Schomakers, J. Schulz, T. Verlage

RWTH Aachen University, III. Physikalisches Institut A, Aachen, Germany

A. Albert, E. Dietz-Laursonn, D. Duchardt, M. Endres, M. Erdmann, S. Erdweg, T. Esch,

R. Fischer, A. Güth, M. Hamer, T. Hebbeker, C. Heidemann, K. Hoepfner, S. Knutzen,

M. Merschmeyer, A. Meyer, P. Millet, S. Mukherjee, M. Olschewski, K. Padeken, T. Pook,

M. Radziej, H. Reithler, M. Rieger, F. Scheuch, D. Teyssier, S. Thüer

RWTH Aachen University, III. Physikalisches Institut B, Aachen, Germany

G. Flügge, B. Kargoll, T. Kress, A. Künsken, J. Lingemann, T. Müller, A. Nehrkorn, A. Nowack, C. Pistone, O. Pooth, A. Stahl ${ }^{13}$

\section{Deutsches Elektronen-Synchrotron, Hamburg, Germany}

M. Aldaya Martin, T. Arndt, C. Asawatangtrakuldee, K. Beernaert, O. Behnke, U. Behrens, A. Bermúdez Martínez, A.A. Bin Anuar, K. Borras ${ }^{14}$, V. Botta, A. Campbell, P. Connor, C. Contreras-Campana, F. Costanza, C. Diez Pardos, G. Eckerlin, D. Eckstein, T. Eichhorn, E. Eren, E. Gallo ${ }^{15}$, J. Garay Garcia, A. Geiser, A. Gizhko, J.M. Grados Luyando, A. Grohsjean, P. Gunnellini, M. Guthoff, A. Harb, J. Hauk, M. Hempel ${ }^{16}$, H. Jung, A. Kalogeropoulos, M. Kasemann, J. Keaveney, C. Kleinwort, I. Korol, D. Krücker, W. Lange, A. Lelek, T. Lenz, J. Leonard, K. Lipka, W. Lohmann ${ }^{16}$, R. Mankel, I.-A. Melzer-Pellmann, A.B. Meyer, G. Mittag, J. Mnich, A. Mussgiller, E. Ntomari, D. Pitzl, A. Raspereza, B. Roland, M. Savitskyi, P. Saxena, R. Shevchenko, S. Spannagel, N. Stefaniuk, G.P. Van Onsem, R. Walsh, Y. Wen, K. Wichmann, C. Wissing, O. Zenaiev

\section{University of Hamburg, Hamburg, Germany}

S. Bein, V. Blobel, M. Centis Vignali, T. Dreyer, E. Garutti, D. Gonzalez, J. Haller, A. Hinzmann, M. Hoffmann, A. Karavdina, R. Klanner, R. Kogler, N. Kovalchuk, S. Kurz, T. Lapsien, I. Marchesini, D. Marconi, M. Meyer, M. Niedziela, D. Nowatschin, F. Pantaleo ${ }^{13}$, T. Peiffer, A. Perieanu, C. Scharf, P. Schleper, A. Schmidt, S. Schumann, J. Schwandt, J. Sonneveld, H. Stadie, G. Steinbrück, F.M. Stober, M. Stöver, H. Tholen, D. Troendle, E. Usai, L. Vanelderen, A. Vanhoefer, B. Vormwald

Institut für Experimentelle Kernphysik, Karlsruhe, Germany

M. Akbiyik, C. Barth, S. Baur, E. Butz, R. Caspart, T. Chwalek, F. Colombo, W. De Boer, A. Dierlamm, B. Freund, R. Friese, M. Giffels, A. Gilbert, D. Haitz, F. Hartmann ${ }^{13}$, 
S.M. Heindl, U. Husemann, F. Kassel ${ }^{13}$, S. Kudella, H. Mildner, M.U. Mozer, Th. Müller, M. Plagge, G. Quast, K. Rabbertz, M. Schröder, I. Shvetsov, G. Sieber, H.J. Simonis,

R. Ulrich, S. Wayand, M. Weber, T. Weiler, S. Williamson, C. Wöhrmann, R. Wolf

Institute of Nuclear and Particle Physics (INPP), NCSR Demokritos, Aghia Paraskevi, Greece

G. Anagnostou, G. Daskalakis, T. Geralis, V.A. Giakoumopoulou, A. Kyriakis, D. Loukas,

I. Topsis-Giotis

National and Kapodistrian University of Athens, Athens, Greece

G. Karathanasis, S. Kesisoglou, A. Panagiotou, N. Saoulidou

National Technical University of Athens, Athens, Greece

K. Kousouris

University of Ioánnina, Ioánnina, Greece

I. Evangelou, C. Foudas, P. Kokkas, S. Mallios, N. Manthos, I. Papadopoulos, E. Paradas, J. Strologas, F.A. Triantis

MTA-ELTE Lendület CMS Particle and Nuclear Physics Group, Eötvös Loránd University, Budapest, Hungary

M. Csanad, N. Filipovic, G. Pasztor, G.I. Veres ${ }^{17}$

Wigner Research Centre for Physics, Budapest, Hungary

G. Bencze, C. Hajdu, D. Horvath ${ }^{18}$, Á. Hunyadi, F. Sikler, V. Veszpremi, A.J. Zsigmond

Institute of Nuclear Research ATOMKI, Debrecen, Hungary

N. Beni, S. Czellar, J. Karancsi ${ }^{19}$, A. Makovec, J. Molnar, Z. Szillasi

Institute of Physics, University of Debrecen, Debrecen, Hungary

M. Bartók ${ }^{17}$, P. Raics, Z.L. Trocsanyi, B. Ujvari

Indian Institute of Science (IISc), Bangalore, India

S. Choudhury, J.R. Komaragiri

National Institute of Science Education and Research, Bhubaneswar, India

S. Bahinipati ${ }^{20}$, S. Bhowmik, P. Mal, K. Mandal, A. Nayak ${ }^{21}$, D.K. Sahoo ${ }^{20}$, N. Sahoo, S.K. Swain

Panjab University, Chandigarh, India

S. Bansal, S.B. Beri, V. Bhatnagar, R. Chawla, N. Dhingra, A.K. Kalsi, A. Kaur, M. Kaur, R. Kumar, P. Kumari, A. Mehta, J.B. Singh, G. Walia

University of Delhi, Delhi, India

Ashok Kumar, Aashaq Shah, A. Bhardwaj, S. Chauhan, B.C. Choudhary, R.B. Garg, S. Keshri, A. Kumar, S. Malhotra, M. Naimuddin, K. Ranjan, R. Sharma

Saha Institute of Nuclear Physics, HBNI, Kolkata, India

R. Bhardwaj, R. Bhattacharya, S. Bhattacharya, U. Bhawandeep, S. Dey, S. Dutt, S. Dutta,

S. Ghosh, N. Majumdar, A. Modak, K. Mondal, S. Mukhopadhyay, S. Nandan, A. Purohit, A. Roy, D. Roy, S. Roy Chowdhury, S. Sarkar, M. Sharan, S. Thakur 
Indian Institute of Technology Madras, Madras, India

P.K. Behera

Bhabha Atomic Research Centre, Mumbai, India

R. Chudasama, D. Dutta, V. Jha, V. Kumar, A.K. Mohanty ${ }^{13}$, P.K. Netrakanti, L.M. Pant, P. Shukla, A. Topkar

Tata Institute of Fundamental Research-A, Mumbai, India

T. Aziz, S. Dugad, B. Mahakud, S. Mitra, G.B. Mohanty, N. Sur, B. Sutar

Tata Institute of Fundamental Research-B, Mumbai, India

S. Banerjee, S. Bhattacharya, S. Chatterjee, P. Das, M. Guchait, Sa. Jain, S. Kumar, M. Maity ${ }^{22}$, G. Majumder, K. Mazumdar, T. Sarkar ${ }^{22}$, N. Wickramage ${ }^{23}$

Indian Institute of Science Education and Research (IISER), Pune, India

S. Chauhan, S. Dube, V. Hegde, A. Kapoor, K. Kothekar, S. Pandey, A. Rane, S. Sharma

Institute for Research in Fundamental Sciences (IPM), Tehran, Iran

S. Chenarani ${ }^{24}$, E. Eskandari Tadavani, S.M. Etesami ${ }^{24}$, M. Khakzad, M. Mohammadi Najafabadi, M. Naseri, S. Paktinat Mehdiabadi ${ }^{25}$, F. Rezaei Hosseinabadi, B. Safarzadeh ${ }^{26}$, M. Zeinali

University College Dublin, Dublin, Ireland

M. Felcini, M. Grunewald

INFN Sezione di Bari ${ }^{a}$, Università di Bari ${ }^{b}$, Politecnico di Bari ${ }^{c}$, Bari, Italy M. Abbrescia ${ }^{a, b}$, C. Calabria ${ }^{a, b}$, A. Colaleo ${ }^{a}$, D. Creanza ${ }^{a, c}$, L. Cristella ${ }^{a, b}$, N. De

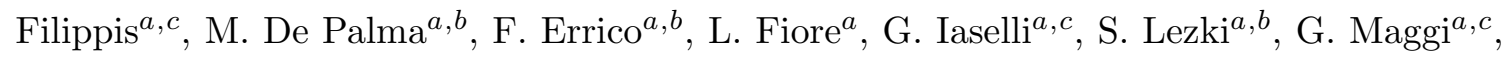
M. Maggi ${ }^{a}$, G. Miniello ${ }^{a, b}$, S. My ${ }^{a, b}$, S. Nuzzo ${ }^{a, b}$, A. Pompili ${ }^{a, b}$, G. Pugliese ${ }^{a, c}$, R. Radogna ${ }^{a, b}$, A. Ranieri ${ }^{a}$, G. Selvaggi ${ }^{a}, b$, A. Sharma ${ }^{a}$, L. Silvestris ${ }^{a, 13}$, R. Venditti ${ }^{a}$, P. Verwilligen ${ }^{a}$

INFN Sezione di Bologna ${ }^{a}$, Università di Bologna ${ }^{b}$, Bologna, Italy

G. Abbiendi ${ }^{a}$, C. Battilana ${ }^{a, b}$, D. Bonacorsi ${ }^{a}, b$, S. Braibant-Giacomelli ${ }^{a, b}$, R. Campanini $^{a, b}$, P. Capiluppi ${ }^{a, b}$, A. Castro ${ }^{a, b}$, F.R. Cavallo ${ }^{a}$, S.S. Chhibra ${ }^{a}$, G. Codispoti ${ }^{a, b}$, M. Cuffiani ${ }^{a, b}$, G.M. Dallavalle ${ }^{a}$, F. Fabbri ${ }^{a}$, A. Fanfani ${ }^{a}, b$, D. Fasanella ${ }^{a, b}$, P. Giacomelli ${ }^{a}$, C. Grandi ${ }^{a}$, L. Guiducci ${ }^{a}, b$, S. Marcellini ${ }^{a}$, G. Masetti ${ }^{a}$, A. Montanari ${ }^{a}$, F.L. Navarria ${ }^{a, b}$, A. Perrotta ${ }^{a}$, A.M. Rossi ${ }^{a, b}$, T. Rovelli ${ }^{a, b}$, G.P. Siroli ${ }^{a, b}$, N. Tosi ${ }^{a}$

INFN Sezione di Catania ${ }^{a}$, Università di Catania ${ }^{b}$, Catania, Italy

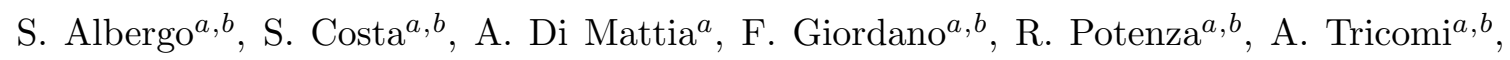
C. Tuve ${ }^{a, b}$

INFN Sezione di Firenze ${ }^{a}$, Università di Firenze ${ }^{b}$, Firenze, Italy

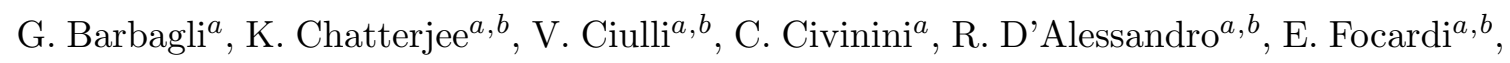

P. Lenzi ${ }^{a, b}$, M. Meschini ${ }^{a}$, S. Paoletti ${ }^{a}$, L. Russo ${ }^{a, 27}$, G. Sguazzoni ${ }^{a}$, D. Strom $^{a}$,

L. Viliani ${ }^{a, b, 13}$ 
INFN Laboratori Nazionali di Frascati, Frascati, Italy

L. Benussi, S. Bianco, F. Fabbri, D. Piccolo, F. Primavera ${ }^{13}$

INFN Sezione di Genova ${ }^{a}$, Università di Genova ${ }^{b}$, Genova, Italy

V. Calvelli ${ }^{a, b}$, F. Ferro $^{a}$, E. Robutti ${ }^{a}$, S. Tosi ${ }^{a, b}$

INFN Sezione di Milano-Bicocca ${ }^{a}$, Università di Milano-Bicocca ${ }^{b}$, Milano, Italy

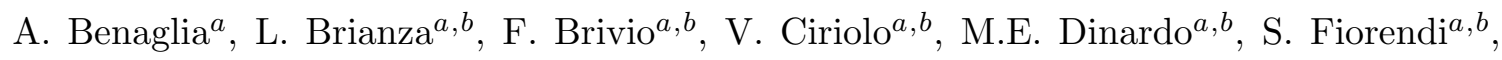

S. Gennai ${ }^{a}$, A. Ghezzi ${ }^{a, b}$, P. Govoni ${ }^{a, b}$, M. Malberti ${ }^{a, b}$, S. Malvezzi ${ }^{a}$, R.A. Manzoni ${ }^{a, b}$,

D. Menasce ${ }^{a}$, L. Moroni ${ }^{a}$, M. Paganoni ${ }^{a, b}$, K. Pauwels ${ }^{a, b}$, D. Pedrini ${ }^{a}$, S. Pigazzini ${ }^{a}, b, 28$,

S. Ragazzi ${ }^{a, b}$, T. Tabarelli de Fatis ${ }^{a, b}$

INFN Sezione di Napoli ${ }^{a}$, Università di Napoli 'Federico II' ${ }^{b}$, Napoli, Italy, Università della Basilicata ${ }^{c}$, Potenza, Italy, Università G. Marconi ${ }^{d}$, Roma, Italy

S. Buontempo ${ }^{a}$, N. Cavallo ${ }^{a, c}$, S. Di Guida ${ }^{a, d, 13}$, F. Fabozzi ${ }^{a, c}$, F. Fienga ${ }^{a, b}$, A.O.M. Iorio ${ }^{a, b}$, W.A. $\operatorname{Khan}^{a}$, L. $\operatorname{Lista}^{a}$, S. Meola ${ }^{a, d, 13}$, P. Paolucci ${ }^{a, 13}$, C. Sciacca ${ }^{a, b}$, F. Thyssen ${ }^{a}$

INFN Sezione di Padova ${ }^{a}$, Università di Padova ${ }^{b}$, Padova, Italy, Università di Trento ${ }^{c}$, Trento, Italy

P. Azzi $^{a, 13}$, N. Bacchetta ${ }^{a}$, L. Benato ${ }^{a, b}$, D. Bisello ${ }^{a, b}$, A. Boletti ${ }^{a, b}$, R. Carlin ${ }^{a, b}$, A. Carvalho Antunes De Oliveira ${ }^{a, b}$, P. Checchia ${ }^{a}$, M. Dall'Osso ${ }^{a, b}$, P. De Castro

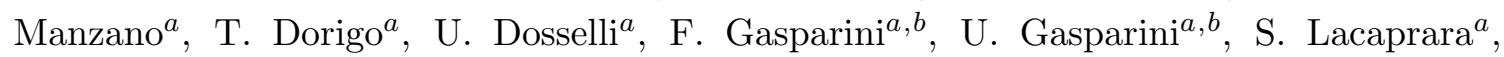
P. Lujan, M. Margoni ${ }^{a, b}$, A.T. Meneguzzo ${ }^{a, b}$, N. Pozzobon ${ }^{a, b}$, P. Ronchese ${ }^{a, b}$, R. $\operatorname{Rossin}^{a, b}$,

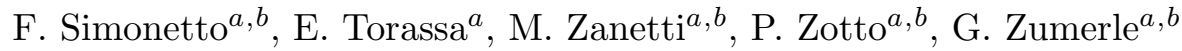

INFN Sezione di Pavia ${ }^{a}$, Università di Pavia ${ }^{b}$, Pavia, Italy

A. Braghieri ${ }^{a}$, A. Magnani ${ }^{a, b}$, P. Montagna ${ }^{a, b}$, S.P. Ratti ${ }^{a, b}$, V. Re ${ }^{a}$, M. Ressegotti, C. Riccardi ${ }^{a, b}$, P. Salvini ${ }^{a}$, I. Vai ${ }^{a}, b$, P. Vitulo ${ }^{a, b}$

INFN Sezione di Perugia ${ }^{a}$, Università di Perugia ${ }^{b}$, Perugia, Italy

L. Alunni Solestizi ${ }^{a, b}$, M. Biasini ${ }^{a, b}$, G.M. Bilei ${ }^{a}$, C. Cecchi $^{a, b}$, D. Ciangottini ${ }^{a, b}$, L. Fanò $^{a, b}$,

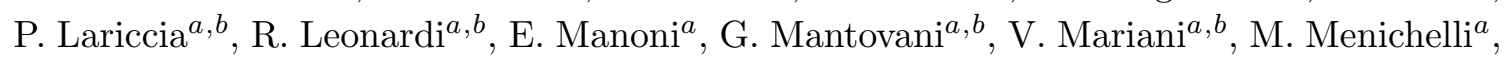
A. $\operatorname{Rossi}^{a, b}$, A. Santocchia ${ }^{a, b}$, D. Spiga ${ }^{a}$

INFN Sezione di Pisa ${ }^{a}$, Università di Pisa ${ }^{b}$, Scuola Normale Superiore di Pisa ${ }^{c}$, Pisa, Italy

K. Androsov ${ }^{a}$, P. Azzurri ${ }^{a, 13}$, G. Bagliesi ${ }^{a}$, J. Bernardini ${ }^{a}$, T. Boccali ${ }^{a}$, L. Borrello,

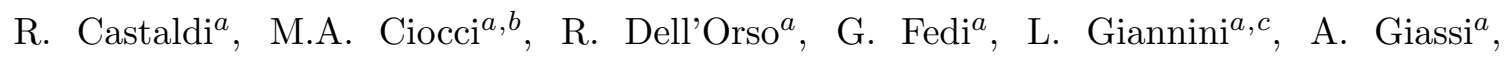
M.T. Grippo ${ }^{a, 27}$, F. Ligabue ${ }^{a, c}$, T. Lomtadze ${ }^{a}$, E. Manca ${ }^{a, c}$, G. Mandorli ${ }^{a, c}$, L. Martini $^{a, b}$,

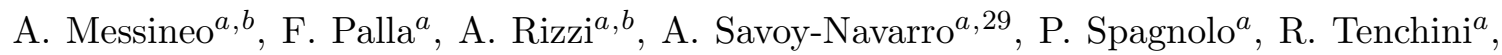
G. Tonelli ${ }^{a, b}$, A. Venturi ${ }^{a}$, P.G. Verdini ${ }^{a}$

INFN Sezione di Roma ${ }^{a}$, Sapienza Università di Roma ${ }^{b}$, Rome, Italy

L. Barone ${ }^{a, b}$, F. Cavallari ${ }^{a}$, M. Cipriani ${ }^{a, b}$, N. Daci ${ }^{a}$, D. Del Re Re $^{a, b, 13}$, E. Di $\operatorname{Marco}^{a, b}$, M. Diemoz ${ }^{a}$, S. Gelli ${ }^{a, b}$, E. Longo ${ }^{a, b}$, F. Margaroli ${ }^{a, b}$, B. Marzocchi ${ }^{a, b}$, 


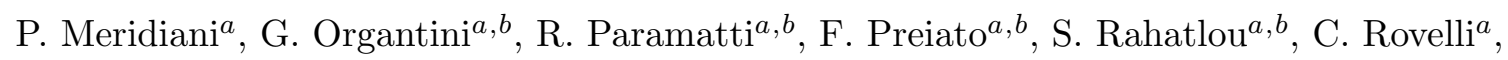
F. Santanastasio ${ }^{a, b}$

INFN Sezione di Torino ${ }^{a}$, Università di Torino ${ }^{b}$, Torino, Italy, Università del Piemonte Orientale ${ }^{c}$, Novara, Italy

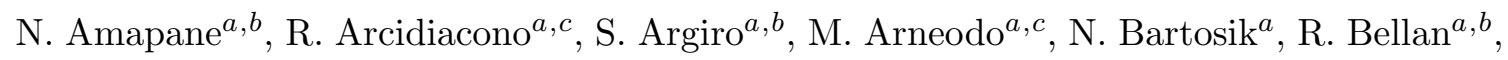

C. Biino ${ }^{a}$, N. Cartiglia ${ }^{a}$, F. Cenna ${ }^{a, b}$, M. Costa ${ }^{a, b}$, R. Covarelli ${ }^{a, b}$, A. Degano ${ }^{a, b}$,

N. $\operatorname{Demaria}^{a}$, B. Kiani ${ }^{a, b}$, C. Mariotti $^{a}$, S. Maselli ${ }^{a}$, E. Migliore ${ }^{a, b}$, V. Monaco ${ }^{a, b}$,

E. Monteil $^{a, b}$, M. Monteno $^{a}$, M.M. Obertino $^{a, b}$, L. Pacher $^{a}, b$, N. Pastrone $^{a}$, M. Pelliccioni $^{a}$,

G.L. Pinna Angioni ${ }^{a, b}$, F. Ravera ${ }^{a, b}$, A. Romero ${ }^{a, b}$, M. Ruspa ${ }^{a, c}$, R. Sacchi ${ }^{a, b}$, K. Shchelina ${ }^{a, b}$, V. Sola ${ }^{a}$, A. Solano ${ }^{a, b}$, A. Staiano ${ }^{a}$, P. Traczyk $^{a, b}$

INFN Sezione di Trieste ${ }^{a}$, Università di Trieste ${ }^{b}$, Trieste, Italy

S. Belforte ${ }^{a}$, M. Casarsa ${ }^{a}$, F. Cossutti ${ }^{a}$, G. Della Ricca ${ }^{a, b}$, A. Zanetti $^{a}$

Kyungpook National University, Daegu, Korea

D.H. Kim, G.N. Kim, M.S. Kim, J. Lee, S. Lee, S.W. Lee, C.S. Moon, Y.D. Oh, S. Sekmen, D.C. Son, Y.C. Yang

Chonbuk National University, Jeonju, Korea

A. Lee

Chonnam National University, Institute for Universe and Elementary Particles, Kwangju, Korea

H. Kim, D.H. Moon, G. Oh

Hanyang University, Seoul, Korea

J.A. Brochero Cifuentes, J. Goh, T.J. Kim

Korea University, Seoul, Korea

S. Cho, S. Choi, Y. Go, D. Gyun, S. Ha, B. Hong, Y. Jo, Y. Kim, K. Lee, K.S. Lee, S. Lee, J. Lim, S.K. Park, Y. Roh

Seoul National University, Seoul, Korea

J. Almond, J. Kim, J.S. Kim, H. Lee, K. Lee, K. Nam, S.B. Oh, B.C. Radburn-Smith, S.h. Seo, U.K. Yang, H.D. Yoo, G.B. Yu

University of Seoul, Seoul, Korea

M. Choi, H. Kim, J.H. Kim, J.S.H. Lee, I.C. Park

Sungkyunkwan University, Suwon, Korea

Y. Choi, C. Hwang, J. Lee, I. Yu

Vilnius University, Vilnius, Lithuania

V. Dudenas, A. Juodagalvis, J. Vaitkus

National Centre for Particle Physics, Universiti Malaya, Kuala Lumpur, Malaysia

I. Ahmed, Z.A. Ibrahim, M.A.B. Md $\mathrm{Ali}^{30}$, F. Mohamad Idris ${ }^{31}$, W.A.T. Wan Abdullah, M.N. Yusli, Z. Zolkapli 
Centro de Investigacion y de Estudios Avanzados del IPN, Mexico City, Mexico Reyes-Almanza, R, Ramirez-Sanchez, G., Duran-Osuna, M. C., H. Castilla-Valdez, E. De La Cruz-Burelo, I. Heredia-De La Cruz ${ }^{32}$, Rabadan-Trejo, R. I., R. Lopez-Fernandez, J. Mejia Guisao, A. Sanchez-Hernandez

\section{Universidad Iberoamericana, Mexico City, Mexico}

S. Carrillo Moreno, C. Oropeza Barrera, F. Vazquez Valencia

Benemerita Universidad Autonoma de Puebla, Puebla, Mexico

I. Pedraza, H.A. Salazar Ibarguen, C. Uribe Estrada

Universidad Autónoma de San Luis Potosí, San Luis Potosí, Mexico

A. Morelos Pineda

University of Auckland, Auckland, New Zealand

D. Krofcheck

University of Canterbury, Christchurch, New Zealand

P.H. Butler

National Centre for Physics, Quaid-I-Azam University, Islamabad, Pakistan

A. Ahmad, M. Ahmad, Q. Hassan, H.R. Hoorani, A. Saddique, M.A. Shah, M. Shoaib, M. Waqas

National Centre for Nuclear Research, Swierk, Poland

H. Bialkowska, M. Bluj, B. Boimska, T. Frueboes, M. Górski, M. Kazana, K. Nawrocki, M. Szleper, P. Zalewski

Institute of Experimental Physics, Faculty of Physics, University of Warsaw, Warsaw, Poland

K. Bunkowski, A. Byszuk ${ }^{33}$, K. Doroba, A. Kalinowski, M. Konecki, J. Krolikowski, M. Misiura, M. Olszewski, A. Pyskir, M. Walczak

Laboratório de Instrumentação e Física Experimental de Partículas, Lisboa, Portugal

P. Bargassa, C. Beirão Da Cruz E Silva, A. Di Francesco, P. Faccioli, B. Galinhas, M. Gallinaro, J. Hollar, N. Leonardo, L. Lloret Iglesias, M.V. Nemallapudi, J. Seixas, G. Strong, O. Toldaiev, D. Vadruccio, J. Varela

Joint Institute for Nuclear Research, Dubna, Russia

S. Afanasiev, P. Bunin, M. Gavrilenko, I. Golutvin, I. Gorbunov, A. Kamenev, V. Karjavin, A. Lanev, A. Malakhov, V. Matveev ${ }^{34,35}$, V. Palichik, V. Perelygin, S. Shmatov, S. Shulha, N. Skatchkov, V. Smirnov, N. Voytishin, A. Zarubin

Petersburg Nuclear Physics Institute, Gatchina (St. Petersburg), Russia Y. Ivanov, V. Kim ${ }^{36}$, E. Kuznetsova ${ }^{37}$, P. Levchenko, V. Murzin, V. Oreshkin, I. Smirnov, V. Sulimov, L. Uvarov, S. Vavilov, A. Vorobyev 
Institute for Nuclear Research, Moscow, Russia

Yu. Andreev, A. Dermenev, S. Gninenko, N. Golubev, A. Karneyeu, M. Kirsanov, N. Krasnikov, A. Pashenkov, D. Tlisov, A. Toropin

Institute for Theoretical and Experimental Physics, Moscow, Russia

V. Epshteyn, V. Gavrilov, N. Lychkovskaya, V. Popov, I. Pozdnyakov, G. Safronov, A. Spiridonov, A. Stepennov, M. Toms, E. Vlasov, A. Zhokin

Moscow Institute of Physics and Technology, Moscow, Russia

T. Aushev, A. Bylinkin ${ }^{35}$

National Research Nuclear University 'Moscow Engineering Physics Institute' (MEPhI), Moscow, Russia

R. Chistov ${ }^{38}$, M. Danilov ${ }^{38}$, P. Parygin, D. Philippov, S. Polikarpov, E. Tarkovskii,

E. Zhemchugov

P.N. Lebedev Physical Institute, Moscow, Russia

V. Andreev, M. Azarkin ${ }^{35}$, I. Dremin ${ }^{35}$, M. Kirakosyan ${ }^{35}$, A. Terkulov

Skobeltsyn Institute of Nuclear Physics, Lomonosov Moscow State University, Moscow, Russia

A. Baskakov, A. Belyaev, E. Boos, M. Dubinin ${ }^{39}$, L. Dudko, A. Ershov, A. Gribushin,

V. Klyukhin, O. Kodolova, I. Lokhtin, I. Miagkov, S. Obraztsov, S. Petrushanko, V. Savrin,

A. Snigirev

Novosibirsk State University (NSU), Novosibirsk, Russia

V. Blinov ${ }^{40}$, Y.Skovpen ${ }^{40}$, D. Shtol ${ }^{40}$

State Research Center of Russian Federation, Institute for High Energy Physics, Protvino, Russia

I. Azhgirey, I. Bayshev, S. Bitioukov, D. Elumakhov, V. Kachanov, A. Kalinin, D. Konstantinov, V. Krychkine, V. Petrov, R. Ryutin, A. Sobol, S. Troshin, N. Tyurin, A. Uzunian, A. Volkov

University of Belgrade, Faculty of Physics and Vinca Institute of Nuclear Sciences, Belgrade, Serbia

P. Adzic ${ }^{41}$, P. Cirkovic, D. Devetak, M. Dordevic, J. Milosevic, V. Rekovic

Centro de Investigaciones Energéticas Medioambientales y Tecnológicas (CIEMAT), Madrid, Spain

J. Alcaraz Maestre, M. Barrio Luna, M. Cerrada, N. Colino, B. De La Cruz, A. Delgado Peris, A. Escalante Del Valle, C. Fernandez Bedoya, J.P. Fernández Ramos, J. Flix, M.C. Fouz, P. Garcia-Abia, O. Gonzalez Lopez, S. Goy Lopez, J.M. Hernandez, M.I. Josa, A. Pérez-Calero Yzquierdo, J. Puerta Pelayo, A. Quintario Olmeda, I. Redondo, L. Romero, M.S. Soares, A. Álvarez Fernández

Universidad Autónoma de Madrid, Madrid, Spain

C. Albajar, J.F. de Trocóniz, M. Missiroli, D. Moran 


\section{Universidad de Oviedo, Oviedo, Spain}

J. Cuevas, C. Erice, J. Fernandez Menendez, I. Gonzalez Caballero, J.R. González Fernández, E. Palencia Cortezon, S. Sanchez Cruz, I. Suárez Andrés, P. Vischia, J.M. Vizan Garcia

\section{Instituto de Física de Cantabria (IFCA), CSIC-Universidad de Cantabria, Santander, Spain}

I.J. Cabrillo, A. Calderon, B. Chazin Quero, E. Curras, J. Duarte Campderros, M. Fernandez, J. Garcia-Ferrero, G. Gomez, A. Lopez Virto, J. Marco, C. Martinez Rivero, P. Martinez Ruiz del Arbol, F. Matorras, J. Piedra Gomez, T. Rodrigo, A. Ruiz-Jimeno, L. Scodellaro, N. Trevisani, I. Vila, R. Vilar Cortabitarte

\section{CERN, European Organization for Nuclear Research, Geneva, Switzerland}

D. Abbaneo, E. Auffray, P. Baillon, A.H. Ball, D. Barney, M. Bianco, P. Bloch, A. Bocci,

C. Botta, T. Camporesi, R. Castello, M. Cepeda, G. Cerminara, E. Chapon, Y. Chen,

D. d'Enterria, A. Dabrowski, V. Daponte, A. David, M. De Gruttola, A. De Roeck, M. Dobson, B. Dorney, T. du Pree, M. Dünser, N. Dupont, A. Elliott-Peisert, P. Everaerts, F. Fallavollita, G. Franzoni, J. Fulcher, W. Funk, D. Gigi, K. Gill, F. Glege, D. Gulhan, P. Harris, J. Hegeman, V. Innocente, P. Janot, O. Karacheban ${ }^{16}$, J. Kieseler, H. Kirschenmann, V. Knünz, A. Kornmayer ${ }^{13}$, M.J. Kortelainen, M. Krammer ${ }^{1}$, C. Lange, P. Lecoq, C. Lourenço, M.T. Lucchini, L. Malgeri, M. Mannelli, A. Martelli, F. Meijers, J.A. Merlin, S. Mersi, E. Meschi, P. Milenovic ${ }^{42}$, F. Moortgat, M. Mulders, H. Neugebauer, S. Orfanelli, L. Orsini, L. Pape, E. Perez, M. Peruzzi, A. Petrilli, G. Petrucciani, A. Pfeiffer, M. Pierini, A. Racz, T. Reis, G. Rolandi ${ }^{43}$, M. Rovere, H. Sakulin, C. Schäfer, C. Schwick, M. Seidel, M. Selvaggi, A. Sharma, P. Silva, P. Sphicas ${ }^{44}$, A. Stakia, J. Steggemann, M. Stoye, M. Tosi, D. Treille, A. Triossi, A. Tsirou, V. Veckalns ${ }^{45}$, M. Verweij, W.D. Zeuner

\section{Paul Scherrer Institut, Villigen, Switzerland}

W. Bertl ${ }^{\dagger}$, L. Caminada 46 , K. Deiters, W. Erdmann, R. Horisberger, Q. Ingram, H.C. Kaestli, D. Kotlinski, U. Langenegger, T. Rohe, S.A. Wiederkehr

Institute for Particle Physics and Astrophysics (IPA), Zurich, Switzerland

F. Bachmair, L. Bäni, P. Berger, L. Bianchini, B. Casal, G. Dissertori, M. Dittmar, M. Donegà, C. Grab, C. Heidegger, D. Hits, J. Hoss, G. Kasieczka, T. Klijnsma, W. Lustermann, B. Mangano, M. Marionneau, M.T. Meinhard, D. Meister, F. Micheli, P. Musella, F. Nessi-Tedaldi, F. Pandolfi, J. Pata, F. Pauss, G. Perrin, L. Perrozzi, M. Quittnat, M. Reichmann, M. Schönenberger, L. Shchutska, V.R. Tavolaro, K. Theofilatos, M.L. Vesterbacka Olsson, R. Wallny, D.H. Zhu

\section{Universität Zürich, Zurich, Switzerland}

T.K. Aarrestad, C. Amsler ${ }^{47}$, M.F. Canelli, A. De Cosa, R. Del Burgo, S. Donato, C. Galloni, T. Hreus, B. Kilminster, J. Ngadiuba, D. Pinna, G. Rauco, P. Robmann, D. Salerno, C. Seitz, Y. Takahashi, A. Zucchetta

\section{National Central University, Chung-Li, Taiwan}

V. Candelise, T.H. Doan, Sh. Jain, R. Khurana, C.M. Kuo, W. Lin, A. Pozdnyakov, S.S. Yu 
National Taiwan University (NTU), Taipei, Taiwan

Arun Kumar, P. Chang, Y. Chao, K.F. Chen, P.H. Chen, F. Fiori, W.-S. Hou, Y. Hsiung, Y.F. Liu, R.-S. Lu, E. Paganis, A. Psallidas, A. Steen, J.f. Tsai

Chulalongkorn University, Faculty of Science, Department of Physics, Bangkok, Thailand

B. Asavapibhop, K. Kovitanggoon, G. Singh, N. Srimanobhas

Çukurova University, Physics Department, Science and Art Faculty, Adana, Turkey

F. Boran, S. Cerci ${ }^{48}$, S. Damarseckin, Z.S. Demiroglu, C. Dozen, I. Dumanoglu, S. Girgis,

G. Gokbulut, Y. Guler, I. $\operatorname{Hos}^{49}$, E.E. Kangal ${ }^{50}$, O. Kara, A. Kayis Topaksu, U. Kiminsu,

M. Oglakci, G. Onengut ${ }^{51}$, K. Ozdemir ${ }^{52}$, D. Sunar $\mathrm{Cerci}^{48}$, B. Talii ${ }^{48}$, S. Turkcapar, I.S. Zorbakir, C. Zorbilmez

Middle East Technical University, Physics Department, Ankara, Turkey

B. Bilin, G. Karapinar ${ }^{53}$, K. Ocalan ${ }^{54}$, M. Yalvac, M. Zeyrek

Bogazici University, Istanbul, Turkey

E. Gülmez, M. Kaya ${ }^{55}$, O. Kaya ${ }^{56}$, S. Tekten, E.A. Yetkin ${ }^{57}$

Istanbul Technical University, Istanbul, Turkey

M.N. Agaras, S. Atay, A. Cakir, K. Cankocak

Institute for Scintillation Materials of National Academy of Science of Ukraine, Kharkov, Ukraine

B. Grynyov

National Scientific Center, Kharkov Institute of Physics and Technology, Kharkov, Ukraine

L. Levchuk, P. Sorokin

University of Bristol, Bristol, United Kingdom

R. Aggleton, F. Ball, L. Beck, J.J. Brooke, D. Burns, E. Clement, D. Cussans, O. Davignon, H. Flacher, J. Goldstein, M. Grimes, G.P. Heath, H.F. Heath, J. Jacob, L. Kreczko, C. Lucas, D.M. Newbold ${ }^{58}$, S. Paramesvaran, A. Poll, T. Sakuma, S. Seif El Nasr-storey, D. Smith, V.J. Smith

Rutherford Appleton Laboratory, Didcot, United Kingdom

K.W. Bell, A. Belyaev ${ }^{59}$, C. Brew, R.M. Brown, L. Calligaris, D. Cieri, D.J.A. Cockerill, J.A. Coughlan, K. Harder, S. Harper, E. Olaiya, D. Petyt, C.H. Shepherd-Themistocleous, A. Thea, I.R. Tomalin, T. Williams

\section{Imperial College, London, United Kingdom}

G. Auzinger, R. Bainbridge, S. Breeze, O. Buchmuller, A. Bundock, S. Casasso, M. Citron,

D. Colling, L. Corpe, P. Dauncey, G. Davies, A. De Wit, M. Della Negra, R. Di Maria,

A. Elwood, Y. Haddad, G. Hall, G. Iles, T. James, R. Lane, C. Laner, L. Lyons, A.-M. Magnan, S. Malik, L. Mastrolorenzo, T. Matsushita, J. Nash, A. Nikitenko ${ }^{6}$, 
V. Palladino, M. Pesaresi, D.M. Raymond, A. Richards, A. Rose, E. Scott, C. Seez, A. Shtipliyski, S. Summers, A. Tapper, K. Uchida, M. Vazquez Acosta ${ }^{60}$, T. Virdee ${ }^{13}$, N. Wardle, D. Winterbottom, J. Wright, S.C. Zenz

\section{Brunel University, Uxbridge, United Kingdom}

J.E. Cole, P.R. Hobson, A. Khan, P. Kyberd, I.D. Reid, P. Symonds, L. Teodorescu, M. Turner

Baylor University, Waco, U.S.A.

A. Borzou, K. Call, J. Dittmann, K. Hatakeyama, H. Liu, N. Pastika, C. Smith

Catholic University of America, Washington DC, U.S.A.

R. Bartek, A. Dominguez

The University of Alabama, Tuscaloosa, U.S.A.

A. Buccilli, S.I. Cooper, C. Henderson, P. Rumerio, C. West

Boston University, Boston, U.S.A.

D. Arcaro, A. Avetisyan, T. Bose, D. Gastler, D. Rankin, C. Richardson, J. Rohlf, L. Sulak, D. Zou

Brown University, Providence, U.S.A.

G. Benelli, D. Cutts, A. Garabedian, J. Hakala, U. Heintz, J.M. Hogan, K.H.M. Kwok, E. Laird, G. Landsberg, Z. Mao, M. Narain, J. Pazzini, S. Piperov, S. Sagir, R. Syarif, D. $\mathrm{Yu}$

University of California, Davis, Davis, U.S.A.

R. Band, C. Brainerd, D. Burns, M. Calderon De La Barca Sanchez, M. Chertok, J. Conway, R. Conway, P.T. Cox, R. Erbacher, C. Flores, G. Funk, M. Gardner, W. Ko, R. Lander, C. Mclean, M. Mulhearn, D. Pellett, J. Pilot, S. Shalhout, M. Shi, J. Smith, M. Squires, D. Stolp, K. Tos, M. Tripathi, Z. Wang

University of California, Los Angeles, U.S.A.

M. Bachtis, C. Bravo, R. Cousins, A. Dasgupta, A. Florent, J. Hauser, M. Ignatenko, N. Mccoll, D. Saltzberg, C. Schnaible, V. Valuev

University of California, Riverside, Riverside, U.S.A.

E. Bouvier, K. Burt, R. Clare, J. Ellison, J.W. Gary, S.M.A. Ghiasi Shirazi, G. Hanson, J. Heilman, P. Jandir, E. Kennedy, F. Lacroix, O.R. Long, M. Olmedo Negrete, M.I. Paneva, A. Shrinivas, W. Si, L. Wang, H. Wei, S. Wimpenny, B. R. Yates

University of California, San Diego, La Jolla, U.S.A.

J.G. Branson, S. Cittolin, M. Derdzinski, R. Gerosa, B. Hashemi, A. Holzner, D. Klein, G. Kole, V. Krutelyov, J. Letts, I. Macneill, M. Masciovecchio, D. Olivito, S. Padhi, M. Pieri, M. Sani, V. Sharma, S. Simon, M. Tadel, A. Vartak, S. Wasserbaech ${ }^{61}$, J. Wood, F. Würthwein, A. Yagil, G. Zevi Della Porta 
University of California, Santa Barbara - Department of Physics, Santa Barbara, U.S.A.

N. Amin, R. Bhandari, J. Bradmiller-Feld, C. Campagnari, A. Dishaw, V. Dutta, M. Franco Sevilla, C. George, F. Golf, L. Gouskos, J. Gran, R. Heller, J. Incandela, S.D. Mullin, A. Ovcharova, H. Qu, J. Richman, D. Stuart, I. Suarez, J. Yoo

California Institute of Technology, Pasadena, U.S.A.

D. Anderson, J. Bendavid, A. Bornheim, J.M. Lawhorn, H.B. Newman, T. Nguyen, C. Pena, M. Spiropulu, J.R. Vlimant, S. Xie, Z. Zhang, R.Y. Zhu

Carnegie Mellon University, Pittsburgh, U.S.A.

M.B. Andrews, T. Ferguson, T. Mudholkar, M. Paulini, J. Russ, M. Sun, H. Vogel, I. Vorobiev, M. Weinberg

University of Colorado Boulder, Boulder, U.S.A.

J.P. Cumalat, W.T. Ford, F. Jensen, A. Johnson, M. Krohn, S. Leontsinis, T. Mulholland, K. Stenson, S.R. Wagner

Cornell University, Ithaca, U.S.A.

J. Alexander, J. Chaves, J. Chu, S. Dittmer, K. Mcdermott, N. Mirman, J.R. Patterson, A. Rinkevicius, A. Ryd, L. Skinnari, L. Soffi, S.M. Tan, Z. Tao, J. Thom, J. Tucker, P. Wittich, M. Zientek

\section{Fermi National Accelerator Laboratory, Batavia, U.S.A.}

S. Abdullin, M. Albrow, G. Apollinari, A. Apresyan, A. Apyan, S. Banerjee, L.A.T. Bauerdick, A. Beretvas, J. Berryhill, P.C. Bhat, G. Bolla ${ }^{\dagger}$, K. Burkett, J.N. Butler, A. Canepa, G.B. Cerati, H.W.K. Cheung, F. Chlebana, M. Cremonesi, J. Duarte, V.D. Elvira, J. Freeman, Z. Gecse, E. Gottschalk, L. Gray, D. Green, S. Grünendahl, O. Gutsche, R.M. Harris, S. Hasegawa, J. Hirschauer, Z. Hu, B. Jayatilaka, S. Jindariani, M. Johnson, U. Joshi, B. Klima, B. Kreis, S. Lammel, D. Lincoln, R. Lipton, M. Liu, T. Liu, R. Lopes De Sá, J. Lykken, K. Maeshima, N. Magini, J.M. Marraffino, S. Maruyama, D. Mason, P. McBride, P. Merkel, S. Mrenna, S. Nahn, V. O’Dell, K. Pedro, O. Prokofyev, G. Rakness, L. Ristori, B. Schneider, E. Sexton-Kennedy, A. Soha, W.J. Spalding, L. Spiegel, S. Stoynev, J. Strait, N. Strobbe, L. Taylor, S. Tkaczyk, N.V. Tran, L. Uplegger, E.W. Vaandering, C. Vernieri, M. Verzocchi, R. Vidal, M. Wang, H.A. Weber, A. Whitbeck University of Florida, Gainesville, U.S.A.

D. Acosta, P. Avery, P. Bortignon, D. Bourilkov, A. Brinkerhoff, A. Carnes, M. Carver, D. Curry, R.D. Field, I.K. Furic, J. Konigsberg, A. Korytov, K. Kotov, P. Ma, K. Matchev, H. Mei, G. Mitselmakher, D. Rank, D. Sperka, N. Terentyev, L. Thomas, J. Wang, S. Wang, J. Yelton

Florida International University, Miami, U.S.A.

Y.R. Joshi, S. Linn, P. Markowitz, J.L. Rodriguez

Florida State University, Tallahassee, U.S.A.

A. Ackert, T. Adams, A. Askew, S. Hagopian, V. Hagopian, K.F. Johnson, T. Kolberg, G. Martinez, T. Perry, H. Prosper, A. Saha, A. Santra, V. Sharma, R. Yohay 
Florida Institute of Technology, Melbourne, U.S.A.

M.M. Baarmand, V. Bhopatkar, S. Colafranceschi, M. Hohlmann, D. Noonan, T. Roy, F. Yumiceva

University of Illinois at Chicago (UIC), Chicago, U.S.A.

M.R. Adams, L. Apanasevich, D. Berry, R.R. Betts, R. Cavanaugh, X. Chen, O. Evdokimov, C.E. Gerber, D.A. Hangal, D.J. Hofman, K. Jung, J. Kamin, I.D. Sandoval Gonzalez, M.B. Tonjes, H. Trauger, N. Varelas, H. Wang, Z. Wu, J. Zhang

The University of Iowa, Iowa City, U.S.A.

B. Bilki ${ }^{62}$, W. Clarida, K. Dilsiz ${ }^{63}$, S. Durgut, R.P. Gandrajula, M. Haytmyradov, V. Khristenko, J.-P. Merlo, H. Mermerkaya ${ }^{64}$, A. Mestvirishvili, A. Moeller, J. Nachtman, H. Ogul ${ }^{65}$, Y. Onel, F. Ozok ${ }^{66}$, A. Penzo, C. Snyder, E. Tiras, J. Wetzel, K. Yi

Johns Hopkins University, Baltimore, U.S.A.

B. Blumenfeld, A. Cocoros, N. Eminizer, D. Fehling, L. Feng, A.V. Gritsan, P. Maksimovic, J. Roskes, U. Sarica, M. Swartz, M. Xiao, C. You

The University of Kansas, Lawrence, U.S.A.

A. Al-bataineh, P. Baringer, A. Bean, S. Boren, J. Bowen, J. Castle, S. Khalil, A. Kropivnitskaya, D. Majumder, W. Mcbrayer, M. Murray, C. Royon, S. Sanders, E. Schmitz, R. Stringer, J.D. Tapia Takaki, Q. Wang

Kansas State University, Manhattan, U.S.A.

A. Ivanov, K. Kaadze, Y. Maravin, A. Mohammadi, L.K. Saini, N. Skhirtladze, S. Toda

Lawrence Livermore National Laboratory, Livermore, U.S.A.

F. Rebassoo, D. Wright

University of Maryland, College Park, U.S.A.

C. Anelli, A. Baden, O. Baron, A. Belloni, B. Calvert, S.C. Eno, C. Ferraioli, N.J. Hadley, S. Jabeen, G.Y. Jeng, R.G. Kellogg, J. Kunkle, A.C. Mignerey, F. Ricci-Tam, Y.H. Shin, A. Skuja, S.C. Tonwar

\section{Massachusetts Institute of Technology, Cambridge, U.S.A.}

D. Abercrombie, B. Allen, V. Azzolini, R. Barbieri, A. Baty, R. Bi, S. Brandt, W. Busza, I.A. Cali, M. D'Alfonso, Z. Demiragli, G. Gomez Ceballos, M. Goncharov, D. Hsu, Y. Iiyama, G.M. Innocenti, M. Klute, D. Kovalskyi, Y.S. Lai, Y.-J. Lee, A. Levin, P.D. Luckey, B. Maier, A.C. Marini, C. Mcginn, C. Mironov, S. Narayanan, X. Niu, C. Paus, C. Roland, G. Roland, J. Salfeld-Nebgen, G.S.F. Stephans, K. Tatar, D. Velicanu, J. Wang, T.W. Wang, B. Wyslouch

University of Minnesota, Minneapolis, U.S.A.

A.C. Benvenuti, R.M. Chatterjee, A. Evans, P. Hansen, S. Kalafut, Y. Kubota, Z. Lesko, J. Mans, S. Nourbakhsh, N. Ruckstuhl, R. Rusack, J. Turkewitz

University of Mississippi, Oxford, U.S.A.

J.G. Acosta, S. Oliveros 
University of Nebraska-Lincoln, Lincoln, U.S.A.

E. Avdeeva, K. Bloom, D.R. Claes, C. Fangmeier, R. Gonzalez Suarez, R. Kamalieddin, I. Kravchenko, J. Monroy, J.E. Siado, G.R. Snow, B. Stieger

State University of New York at Buffalo, Buffalo, U.S.A.

M. Alyari, J. Dolen, A. Godshalk, C. Harrington, I. Iashvili, D. Nguyen, A. Parker, S. Rappoccio, B. Roozbahani

Northeastern University, Boston, U.S.A.

G. Alverson, E. Barberis, A. Hortiangtham, A. Massironi, D.M. Morse, D. Nash, T. Orimoto, R. Teixeira De Lima, D. Trocino, D. Wood

Northwestern University, Evanston, U.S.A.

S. Bhattacharya, O. Charaf, K.A. Hahn, N. Mucia, N. Odell, B. Pollack, M.H. Schmitt, K. Sung, M. Trovato, M. Velasco

University of Notre Dame, Notre Dame, U.S.A.

N. Dev, M. Hildreth, K. Hurtado Anampa, C. Jessop, D.J. Karmgard, N. Kellams, K. Lannon, N. Loukas, N. Marinelli, F. Meng, C. Mueller, Y. Musienko ${ }^{34}$, M. Planer, A. Reinsvold, R. Ruchti, G. Smith, S. Taroni, M. Wayne, M. Wolf, A. Woodard

The Ohio State University, Columbus, U.S.A.

J. Alimena, L. Antonelli, B. Bylsma, L.S. Durkin, S. Flowers, B. Francis, A. Hart, C. Hill, W. Ji, B. Liu, W. Luo, D. Puigh, B.L. Winer, H.W. Wulsin

Princeton University, Princeton, U.S.A.

S. Cooperstein, O. Driga, P. Elmer, J. Hardenbrook, P. Hebda, S. Higginbotham, D. Lange, J. Luo, D. Marlow, K. Mei, I. Ojalvo, J. Olsen, C. Palmer, P. Piroué, D. Stickland, C. Tully

University of Puerto Rico, Mayaguez, U.S.A.

S. Malik, S. Norberg

Purdue University, West Lafayette, U.S.A.

A. Barker, V.E. Barnes, S. Das, S. Folgueras, L. Gutay, M.K. Jha, M. Jones, A.W. Jung, A. Khatiwada, D.H. Miller, N. Neumeister, C.C. Peng, J.F. Schulte, J. Sun, F. Wang, W. Xie

Purdue University Northwest, Hammond, U.S.A.

T. Cheng, N. Parashar, J. Stupak

Rice University, Houston, U.S.A.

A. Adair, B. Akgun, Z. Chen, K.M. Ecklund, F.J.M. Geurts, M. Guilbaud, W. Li, B. Michlin, M. Northup, B.P. Padley, J. Roberts, J. Rorie, Z. Tu, J. Zabel

University of Rochester, Rochester, U.S.A.

A. Bodek, P. de Barbaro, R. Demina, Y.t. Duh, T. Ferbel, M. Galanti, A. Garcia-Bellido, J. Han, O. Hindrichs, A. Khukhunaishvili, K.H. Lo, P. Tan, M. Verzetti

The Rockefeller University, New York, U.S.A.

R. Ciesielski, K. Goulianos, C. Mesropian 
Rutgers, The State University of New Jersey, Piscataway, U.S.A.

A. Agapitos, J.P. Chou, Y. Gershtein, T.A. Gómez Espinosa, E. Halkiadakis, M. Heindl,

E. Hughes, S. Kaplan, R. Kunnawalkam Elayavalli, S. Kyriacou, A. Lath, R. Montalvo, K. Nash, M. Osherson, H. Saka, S. Salur, S. Schnetzer, D. Sheffield, S. Somalwar, R. Stone, S. Thomas, P. Thomassen, M. Walker

University of Tennessee, Knoxville, U.S.A.

A.G. Delannoy, M. Foerster, J. Heideman, G. Riley, K. Rose, S. Spanier, K. Thapa

Texas A\&M University, College Station, U.S.A.

O. Bouhali ${ }^{67}$, A. Castaneda Hernandez ${ }^{67}$, A. Celik, M. Dalchenko, M. De Mattia, A. Delgado, S. Dildick, R. Eusebi, J. Gilmore, T. Huang, T. Kamon ${ }^{68}$, R. Mueller, Y. Pakhotin, R. Patel, A. Perloff, L. Perniè, D. Rathjens, A. Safonov, A. Tatarinov, K.A. Ulmer

\section{Texas Tech University, Lubbock, U.S.A.}

N. Akchurin, J. Damgov, F. De Guio, P.R. Dudero, J. Faulkner, E. Gurpinar, S. Kunori, K. Lamichhane, S.W. Lee, T. Libeiro, T. Peltola, S. Undleeb, I. Volobouev, Z. Wang

Vanderbilt University, Nashville, U.S.A.

S. Greene, A. Gurrola, R. Janjam, W. Johns, C. Maguire, A. Melo, H. Ni, P. Sheldon, S. Tuo, J. Velkovska, Q. Xu

University of Virginia, Charlottesville, U.S.A.

M.W. Arenton, P. Barria, B. Cox, R. Hirosky, A. Ledovskoy, H. Li, C. Neu, T. Sinthuprasith, Y. Wang, E. Wolfe, F. Xia

Wayne State University, Detroit, U.S.A.

R. Harr, P.E. Karchin, J. Sturdy, S. Zaleski

University of Wisconsin - Madison, Madison, WI, U.S.A.

M. Brodski, J. Buchanan, C. Caillol, S. Dasu, L. Dodd, S. Duric, B. Gomber, M. Grothe, M. Herndon, A. Hervé, U. Hussain, P. Klabbers, A. Lanaro, A. Levine, K. Long, R. Loveless, G.A. Pierro, G. Polese, T. Ruggles, A. Savin, N. Smith, W.H. Smith, D. Taylor, N. Woods

$\dagger$ : Deceased

1: Also at Vienna University of Technology, Vienna, Austria

2: Also at State Key Laboratory of Nuclear Physics and Technology, Peking University, Beijing, China

3: Also at Universidade Estadual de Campinas, Campinas, Brazil

4: Also at Universidade Federal de Pelotas, Pelotas, Brazil

5: Also at Université Libre de Bruxelles, Bruxelles, Belgium

6: Also at Institute for Theoretical and Experimental Physics, Moscow, Russia

7: Also at Joint Institute for Nuclear Research, Dubna, Russia

8: Now at Cairo University, Cairo, Egypt

9: Also at Zewail City of Science and Technology, Zewail, Egypt

10: Also at Université de Haute Alsace, Mulhouse, France 
11: Also at Skobeltsyn Institute of Nuclear Physics, Lomonosov Moscow State University, Moscow, Russia

12: Also at Tbilisi State University, Tbilisi, Georgia

13: Also at CERN, European Organization for Nuclear Research, Geneva, Switzerland

14: Also at RWTH Aachen University, III. Physikalisches Institut A, Aachen, Germany

15: Also at University of Hamburg, Hamburg, Germany

16: Also at Brandenburg University of Technology, Cottbus, Germany

17: Also at MTA-ELTE Lendület CMS Particle and Nuclear Physics Group, Eötvös Loránd University, Budapest, Hungary

18: Also at Institute of Nuclear Research ATOMKI, Debrecen, Hungary

19: Also at Institute of Physics, University of Debrecen, Debrecen, Hungary

20: Also at Indian Institute of Technology Bhubaneswar, Bhubaneswar, India

21: Also at Institute of Physics, Bhubaneswar, India

22: Also at University of Visva-Bharati, Santiniketan, India

23: Also at University of Ruhuna, Matara, Sri Lanka

24: Also at Isfahan University of Technology, Isfahan, Iran

25: Also at Yazd University, Yazd, Iran

26: Also at Plasma Physics Research Center, Science and Research Branch, Islamic Azad University, Tehran, Iran

27: Also at Università degli Studi di Siena, Siena, Italy

28: Also at INFN Sezione di Milano-Bicocca; Università di Milano-Bicocca, Milano, Italy

29: Also at Purdue University, West Lafayette, U.S.A.

30: Also at International Islamic University of Malaysia, Kuala Lumpur, Malaysia

31: Also at Malaysian Nuclear Agency, MOSTI, Kajang, Malaysia

32: Also at Consejo Nacional de Ciencia y Tecnología, Mexico city, Mexico

33: Also at Warsaw University of Technology, Institute of Electronic Systems, Warsaw, Poland

34: Also at Institute for Nuclear Research, Moscow, Russia

35: Now at National Research Nuclear University 'Moscow Engineering Physics Institute' (MEPhI), Moscow, Russia

36: Also at St. Petersburg State Polytechnical University, St. Petersburg, Russia

37: Also at University of Florida, Gainesville, U.S.A.

38: Also at P.N. Lebedev Physical Institute, Moscow, Russia

39: Also at California Institute of Technology, Pasadena, U.S.A.

40: Also at Budker Institute of Nuclear Physics, Novosibirsk, Russia

41: Also at Faculty of Physics, University of Belgrade, Belgrade, Serbia

42: Also at University of Belgrade, Faculty of Physics and Vinca Institute of Nuclear Sciences, Belgrade, Serbia

43: Also at Scuola Normale e Sezione dell'INFN, Pisa, Italy

44: Also at National and Kapodistrian University of Athens, Athens, Greece

45: Also at Riga Technical University, Riga, Latvia

46: Also at Universität Zürich, Zurich, Switzerland

47: Also at Stefan Meyer Institute for Subatomic Physics (SMI), Vienna, Austria

48: Also at Adiyaman University, Adiyaman, Turkey

49: Also at Istanbul Aydin University, Istanbul, Turkey

50: Also at Mersin University, Mersin, Turkey

51: Also at Cag University, Mersin, Turkey

52: Also at Piri Reis University, Istanbul, Turkey

53: Also at Izmir Institute of Technology, Izmir, Turkey 
54: Also at Necmettin Erbakan University, Konya, Turkey

55: Also at Marmara University, Istanbul, Turkey

56: Also at Kafkas University, Kars, Turkey

57: Also at Istanbul Bilgi University, Istanbul, Turkey

58: Also at Rutherford Appleton Laboratory, Didcot, United Kingdom

59: Also at School of Physics and Astronomy, University of Southampton, Southampton, United Kingdom

60: Also at Instituto de Astrofísica de Canarias, La Laguna, Spain

61: Also at Utah Valley University, Orem, U.S.A.

62: Also at Beykent University, Istanbul, Turkey

63: Also at Bingol University, Bingol, Turkey

64: Also at Erzincan University, Erzincan, Turkey

65: Also at Sinop University, Sinop, Turkey

66: Also at Mimar Sinan University, Istanbul, Istanbul, Turkey

67: Also at Texas A\&M University at Qatar, Doha, Qatar

68: Also at Kyungpook National University, Daegu, Korea 